The High-Field Penning-Malmberg Trap: Confinement Properties and Use in Positron Accumulation

\author{
Jay Harrison Hartley \\ (Ph.D. Thesis)
}

September 1997 


\section{DISCLAIMER}

This document was prepared as account of work sponsored by an agency of the United States Government. Neither the United States Government nor the University of Colifornia nor any of their employees, makes any warranty, express or implied, or aseumes any legal liability or responaibility for the accuracy, completeness, or usefulness of any information, apparatus, product, or process disclosed, or represents that its use would not infringe privately owned rights. Reference herein to any specific commercial product, process, or service by trade name, tredemark, manufacturer, or other wise, does not necesearily constitute or imply its endorsement, recommendation, or favoring by the United States Government or the University of California. The views and optnions of authors expreseed herein do not necesearily state or reflect those of the United States Government or the University of Calfornia, and shall not be used for advertising or product endorsement purpoees.

This report has been reproduced

directly from the best available copy.

Available to DOE and DOE contractors from the Office of Scientific and Technical Information

P.O. Box 62, Oak Ridge, TN 37831

Prices available from (615) 576-8401, FTS 626-8401

Available to the public from the

National Technical Information Service

U.S. Department of Commerce

5285 Port Royal Rd.,

Springfield, VA 22161

Work performed under the auspices of the U.S. Department of Energy by Lawrence Livermore National Laboratory under Contract W-7405-ENG-48. 


\title{
The High-Field Penning-Malmberg Trap: Confinement Properties and Use in Positron Accumulation
}

\author{
Jay Harrison Hartley
}

(Ph.D. Thesis)

September 1997

\section{LAWRENCE LIVERMORE NATIONAL LABORATORY \\ University of California - Livermore, California - 94551}


The High-Field Penning-Malmberg Trap: Confinement Properties and Use in Positron Accumulation

\section{By}

JAY HARRISON HARTLEY

B.A. (Williams College, MA) 1990

M.S. (University of California, Davis) 1992

\section{DISSERTATION}

Submitted in partial satisfaction of the requirements for the degree of

\section{DOCTOR OF PHIILOSOPHY}

in

Applied Science

in the

OFFICE OF GRADUATE STUDIES

of the

UNIVERSITY OF CALIFORNIA

DAVIS

Approved:

Prof. David Hwang

Dr. Thomas E. Cowan

Prof. Thomas Cahill

Committee in Charge

1997 


\begin{abstract}
This dissertation reports on the development of the $60 \mathrm{kG}$ cryogenic positron trap at Lawrence Livermore National Laboratory, and compares the trap's confinement properties with other nonneutral plasma devices. The device is designed for the accumulation of up to $2 \times 10^{9}$ positrons from a linear-accelerator source. This positron plasma could then be used in Bhabha scattering experiments. Initial efforts at time-of-flight accumulation of positrons from the accelerator show rapid $(\sim 100 \mathrm{~ms})$ deconfinement, inconsistent with the long electron lifetimes. Several possible deconfinement mechanisms have been explored, including annihilation on residual gas, injection heating, rf noise from the accelerator, magnet field curvature, and stray fields. Detailed studies of electron confinement demonstrate that the empirical scaling law used to design the trap cannot be extrapolated into the parameter regime of this device. Several possible methods for overcoming these limitations are presented.
\end{abstract}




\section{Acknowledgements}

There is someone to thank for every page of this dissertation, and I regret that I do not have the time, space, or memory to acknowledge them all. It is easy in graduate school to feel alone, abandoned, and sorry for oneself, but I could not have completed this project without the support and effort of many, many others.

I want to thank my research advisor, Dr. Tom Cowan. His love of science is infectious, and his integrity and drive are inspiring. The lessons I have learned from Tom will serve me well the rest of my career. I would also like to thank Prof. David Hwang for his physics insights and support when I was struggling to make sense of this project, and Prof. Thomas Cahill for his patience, encouragement, and general good will.

The support of technicians is crucial to the success of any graduate student, and I will be eternally grateful to Bill Patterson, Herm Koberle, Hank Glauser, Nick Skluff, Bob Hall, and the many others who have helped keep the LLNL linac operating and took the time to 
help and advise me, even when I didn't have an account number for them. I also want to thank Archietta Johnson, Lisa Swayne, Don Hoffman, and the rest of the Applied Science and Student Employee staff for helping me navigate the administrative jungles of the University of California and LLNL.

I warmly acknowledge the work of Rajeev Rohatgi and Bret Beck in designing the positron trap and making it operational, and Rich Howell in creating the best positron source in the country and keeping it alive against all odds. I also received important help and advice from everyone in the nonneutral research groups at UC San Diego and UC Berkeley. This work builds on, and I hope complements, their impressive achievements.

As in everything I do, the importance of my parents, Rev. James and Virginia Hartley, cannot be overstated. They have given me a lifetime of encouragement and love that is beyond value. Finally, I must thank my loving wife, Cory, and sons Xanth and Matthew. They have suffered the most these past few years, and I owe them a great debt for their patience, understanding, and steadfast love. 


\section{Table of Contents}

1 Introduction $\ldots \ldots \ldots \ldots \ldots \ldots \ldots \ldots \ldots \ldots \ldots \ldots \ldots \ldots \ldots \ldots \ldots \ldots$

1.1 Motivation $\ldots \ldots \ldots \ldots \ldots \ldots \ldots \ldots \ldots \ldots \ldots \ldots \ldots \ldots \ldots \ldots \ldots$

1.2 Design of Bhabha Scattering Experiment $\ldots \ldots \ldots 3$

1.2.1 Positron Heating $\ldots \ldots \ldots \ldots \ldots \ldots \ldots \ldots \ldots, 4$

1.2.2 Scattering Rate $\ldots \ldots \ldots \ldots \ldots \ldots \ldots \ldots \ldots$

1.2.3 Accumulation Rate $\ldots \ldots \ldots \ldots \ldots \ldots \ldots \ldots .9$

1.3 Nonneutral Plasma Confinement $\ldots \ldots \ldots \ldots \ldots \ldots 12$

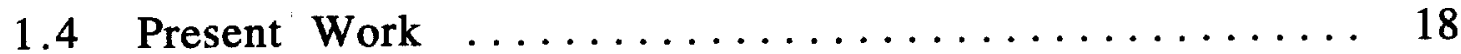

2 Experimental Apparatus $\ldots \ldots \ldots \ldots \ldots \ldots \ldots \ldots \ldots \ldots 22$

2.1 Overview of the LLNL Nonneutral Plasma Trap ..... 22

2.2 Positron Source $\ldots \ldots \ldots \ldots \ldots \ldots \ldots \ldots \ldots \ldots \ldots \ldots \ldots \ldots \ldots$

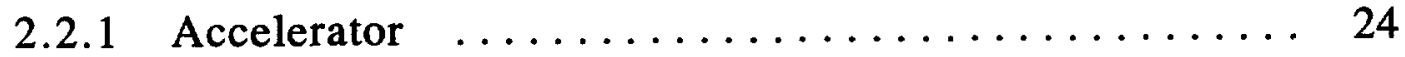

2.2.2 Positron Converter and Moderator ......... 25

2.2.3 Transport beamline ................ 27

2.3 Electron source $\ldots \ldots \ldots \ldots \ldots \ldots \ldots \ldots \ldots \ldots \ldots \ldots$

2.4 Penning-Malmberg Trap $\ldots \ldots \ldots \ldots \ldots \ldots \ldots \ldots \ldots . \ldots \ldots$

2.4 .1 Electrodes $\ldots \ldots \ldots \ldots \ldots \ldots \ldots \ldots \ldots \ldots \ldots \ldots$

2.4.2 Magnet $\ldots \ldots \ldots \ldots \ldots \ldots \ldots \ldots \ldots \ldots \ldots \ldots, 40$

2.5 Diagnostics $\ldots \ldots \ldots \ldots \ldots \ldots \ldots \ldots \ldots \ldots \ldots \ldots \ldots$

2.5.1 Imaging System $\ldots \ldots \ldots \ldots \ldots \ldots \ldots \ldots \ldots \ldots 44$

2.5.2 Gamma-ray Detectors $\ldots \ldots \ldots \ldots \ldots \ldots \ldots \ldots \ldots$

2.5.3 Diocotron Motion $\ldots \ldots \ldots \ldots \ldots \ldots \ldots \ldots \ldots \ldots$ 
2.5.4 Temperature Measurement $\ldots \ldots \ldots \ldots \ldots \ldots \ldots 59$

2.6 Control Electronics $\ldots \ldots \ldots \ldots \ldots \ldots \ldots \ldots \ldots \ldots \ldots$

2.7 Trap Operation $\ldots \ldots \ldots \ldots \ldots \ldots \ldots \ldots \ldots \ldots \ldots \ldots$

2.7.1 Continuous Electron Source Injection $\ldots \ldots \ldots \ldots 63$

2.7.2 Pulsed Injection $\ldots \ldots \ldots \ldots \ldots \ldots \ldots \ldots \ldots \ldots 6$

3 Positron Experiments $\ldots \ldots \ldots \ldots \ldots \ldots \ldots \ldots \ldots \ldots \ldots$

3.1 Data Collection and Analysis ............. 67

3.2 Basic TOF Accumulation and Confinement ....... 69

3.3 Effect of Residual Gas Pressure $\ldots \ldots \ldots \ldots \ldots \ldots \ldots$

3.4 Positron Heating $\ldots \ldots \ldots \ldots \ldots \ldots \ldots \ldots \ldots \ldots . \ldots \ldots$

3.5 Low-Energy Injection into a Short Trap $\ldots \ldots \ldots \ldots 83$

3.6 New Trapping Schemes $\ldots \ldots \ldots \ldots \ldots \ldots \ldots \ldots \ldots 87$

3.7 Positron summary $\ldots \ldots \ldots \ldots \ldots \ldots \ldots \ldots \ldots \ldots$

4 Electron Confinement $\ldots \ldots \ldots \ldots \ldots \ldots \ldots \ldots \ldots \ldots \ldots . \ldots \ldots$

4.1 Data Collection and Analysis ............. 93

4.1.1 Trap Operation $\ldots \ldots \ldots \ldots \ldots \ldots \ldots \ldots \ldots . \ldots 3$

4.1.2 Length Calculations $\ldots \ldots \ldots \ldots \ldots \ldots \ldots \ldots \ldots 94$

4.1.3 Temperature $\ldots \ldots \ldots \ldots \ldots \ldots \ldots \ldots \ldots . \ldots 8$

4.1.4 Density Measurement .............. 102

4.1.5 Lifetime Calculation $\ldots \ldots \ldots \ldots \ldots \ldots \ldots \ldots . \ldots$

4.2 Half-Life Analysis $\ldots \ldots \ldots \ldots \ldots \ldots \ldots \ldots \ldots \ldots \ldots 11$

4.2 .1 Length $\ldots \ldots \ldots \ldots \ldots \ldots \ldots \ldots \ldots \ldots \ldots \ldots, 111$

4.2.2 Magnetic Field $\ldots \ldots \ldots \ldots \ldots \ldots \ldots \ldots \ldots \ldots 112$

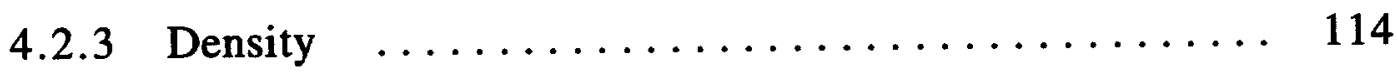

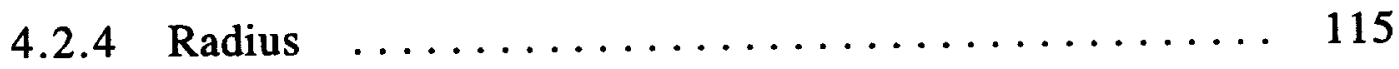

4.2.5 Comparison to $(\mathrm{B} / \mathrm{L})^{2}$ and Bounce-Resonance Theory 118 
4.3 Dynamic Evolution Analysis $\ldots \ldots \ldots \ldots \ldots \ldots \ldots \ldots 122$

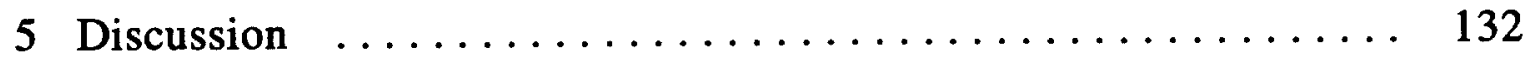

5.1 Conclusions $\ldots \ldots \ldots \ldots \ldots \ldots \ldots \ldots \ldots \ldots \ldots \ldots \ldots \ldots \ldots \ldots$

5.2 Positron Improvements $\ldots \ldots \ldots \ldots \ldots \ldots \ldots \ldots \ldots$

5.3 Electron Improvements $\ldots \ldots \ldots \ldots \ldots \ldots \ldots \ldots \ldots \ldots$

5.4 Future Experiments $\ldots \ldots \ldots \ldots \ldots \ldots \ldots \ldots \ldots \ldots . \ldots \ldots$

Appendix A Particle Deconfinement . . . . . . . . . . . 142

A.1 Transport Theories $\ldots \ldots \ldots \ldots \ldots \ldots \ldots \ldots \ldots \ldots 142$

A.2 Electron Experiments $\ldots \ldots \ldots \ldots \ldots \ldots \ldots \ldots \ldots \ldots 147$

A.3 Positron Experiments $\ldots \ldots \ldots \ldots \ldots \ldots \ldots \ldots \ldots \ldots 153$

Appendix B Positron Interactions with Residual Gases $\ldots \ldots \ldots 156$

Appendix C Single-Particle Ray Tracing ............. 159

C.1 Numerical Calculations $\ldots \ldots \ldots \ldots \ldots \ldots \ldots \ldots \ldots$

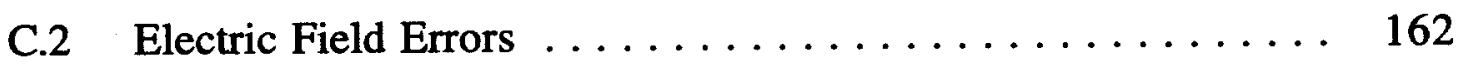

C.3 Magnetic Field Errors $\ldots \ldots \ldots \ldots \ldots \ldots \ldots \ldots \ldots \ldots$

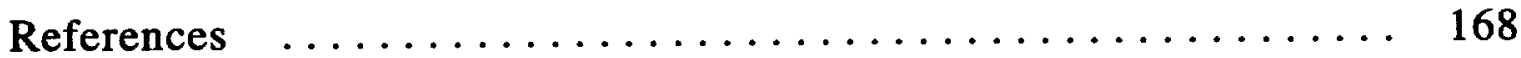




\section{Disclaimer}

This document was prepared as an account of work sponsored by an agency of the United States Government. Neither the United States Government nor the University of California nor any of their employees, makes any warranty, express or implied, or assumes any legal liability or responsibility for the accuracy, completeness, or usefulness of any information, apparatus, product, or process disclosed, or represents that its use would not infringe privately owned rights. Reference herein to any specific commercial product, process, or service by trade name, trademark, manufacturer, or otherwise, does not necessarily constitute or imply its endorsement, recommendation, or favoring by the United States Government or the University of California. The views and opinions of authors expressed herein do not necessarily state or reflect those of the United States Government or the University of California, and shall not be used for advertising or product endorsement purposes.

Work performed under the auspices of the U.S. Department of Energy by Lawrence Livermore National Laboratory under Contract W7405-ENG-48. 


\section{$1 \quad$ Introduction}

\subsection{Motivation}

The development of a high-density positron plasma at Lawrence Livermore National Laboratory (LLNL) was originally driven by the need for a positron gas target for nuclear physics experiments. Heavy-ion collision experiments by the EPOS (Electron-POsitron Spectrometer) collaboration at GSI Darmstadt discovered unexpected peaks in the energy spectra of emitted positrons $[1,2]$. Further studies demonstrated that these monoenergetic positrons were associated with the apparent back-to-back emission of electrons at the same energy $[3,4,5]$. The narrowness of the peaks and their presence in collisions with varying nuclei $(\mathrm{U}+\mathrm{Th}$ and $\mathrm{U}+\mathrm{Ta})$ suggested the formation of unknown neutral objects, with lifetimes of $10^{-19}$. $10^{-9} \mathrm{~s}$ and masses of $1.5-2 \mathrm{MeV} / \mathrm{c}^{2}$, that decayed into the observed electron-positron pairs. 
One way to explore this possibility was to search for the reverse process by forming the unknown object in positron-electron collisions. Experiments were performed looking for resonances in the elastic positron-electron (Bhabha) scattering spectrum utilizing positron beams incident on thin foil targets [6,7]. The momentum of the bound electrons resulted in a large spread in the center-of-mass collision energy, which limited the sensitivity of these measurements. Experiments using thicker targets to increase the event rate and recoil-shadow techniques to limit the measured phase space [8] gave results that were highly sensitive to the model of the interaction between the hypothetical neutral object and the thick target and beam dump material.

In order to achieve greater sensitivity with a thin target, an experiment was designed using a cold, magnetically confined gas target of positrons $[9,10]$ and an intense $(\sim 10 \mu \mathrm{A})$, variable energy electron beam from a $3 \mathrm{MeV}$ Pelletron accelerator. Positrons are accumulated in a magnetic trap from a linear accelerator (linac) source. A schematic of the proposed experiment is shown in Fig. 1.1. In order to achieve reasonable counting rates in the scattering 


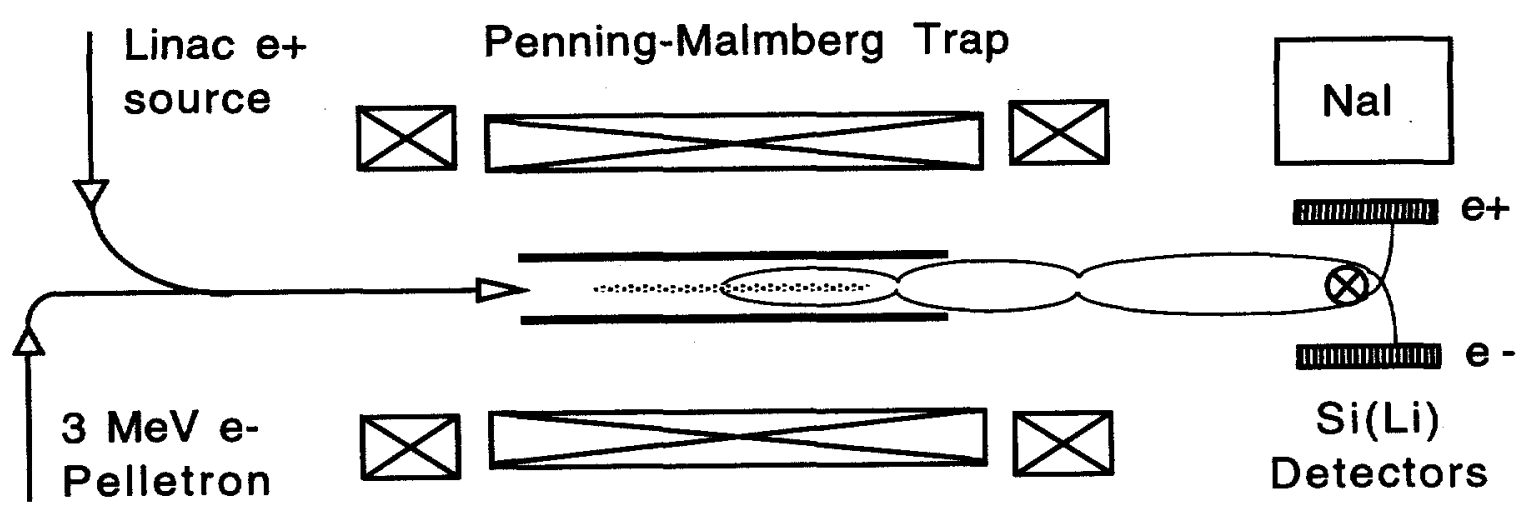

Figure 1.1 Schematic of Bhabha scattering experiment. Positrons (e+) are accumulated in the trap. The electron (e-) beam scatters in the e+ target. Scattered particles are separated by a transverse permanent magnet in the fringe field of the solenoid.

experiment, a total of approximately $2 \times 10^{9}$ positrons at a density of $10^{10} \mathrm{e}^{+} / \mathrm{cm}^{3}$ must be confined for $\approx 300 \mathrm{~s}$. Such confinement properties had been achieved previously with electrons in a cryogenic nonneutral Penning-Malmberg trap [11].

\subsection{Design of Bhabha Scattering Experiment [12]}

The Bhabha scattering experiment must be able to detect a $1 \%$ resonant enhancement of the scattering cross section with an energy width $\approx 1 \mathrm{keV}$. In order to see a $1 \%$ signal at a $3 \sigma$ confidence level, Poisson statistics requires 


$$
\sqrt{N} / N \geq 0.003
$$

or $N \geq 10^{5}$ detected events at each collision energy. A rate of $1 \mathrm{~s}^{-1}$

requires 2 days per energy point, allowing the experiment to be performed on a one year timescale. The positron plasma density and length, the incident electron beam intensity, and the detector efficiency have to be large enough to provide this rate. This constraint has to be balanced with the confinement properties of the positron plasma and the heating that results from the beam-plasma interaction.

\subsubsection{Positron Heating}

The positron temperature should be $\leq 100 \mathrm{~K}$ in order to minimize the center-of-mass energy spread of the collision. Transformed to the center-of-mass frame $(\gamma=1.85), 100 \mathrm{~K}$ is equivalent to the $200 \mathrm{eV}$ peak-to-peak (29 eV rms) lab-frame spread of the $3 \mathrm{MeV}$ electron beam. The equilibrium temperature is determined by balancing positron heating, due primarily to electron energy loss, with cooling by cyclotron radiation. 
A test particle travelling through matter loses energy at the rate,

$$
\frac{d E}{d x}=2 \pi n \int_{b_{\min }}^{b_{\max }} \Delta E(b) b d b,
$$

where $n$ is the particle density, $b$ is the impact parameter, and $\Delta E$ is the amount of energy lost in a single collision [13]. For impact parameters less than the Debye shielding length,

$$
\lambda_{D} \equiv \sqrt{\frac{k T}{4 \pi n e^{2}}} \approx 74.3 \mu \mathrm{m}\left(\frac{T}{1 \mathrm{eV}}\right)^{1 / 2}\left(\frac{n}{10^{10} \mathrm{~cm}^{-3}}\right)^{-1 / 2}
$$

the two-body collision with a screened potential can be used. For $b>\lambda_{D}$, energy transfer to collective modes of the plasma becomes significant. Taking these effects into account, the total energy loss of a relativistic electron $\left(\beta=v / c, \gamma=\left(1-\beta^{2}\right)^{-1 / 2}\right)$ passing through the positron plasma is approximately,

$$
\frac{d E}{d x} \sim\left(\frac{e}{\beta c}\right)^{2} \omega_{p}^{2} \ln \left(\frac{r_{p}}{b_{\min }}\right),
$$

where $r_{p}$ is the plasma radius, $\omega_{p}^{2}=4 \pi n e^{2} / m$ is the plasma frequency, and the minimum impact parameter is, 


$$
b_{\min }=\frac{\hbar}{m c} \sqrt{\frac{2}{\gamma-1}}
$$

Given a current of electrons $I_{e^{-}}$through a plasma of length $L$, the heating per positron will be,

$$
\frac{d E}{d t}=\frac{d E}{d x} \frac{L I_{e^{-}}}{e N_{e^{+}}} \approx 0.21 \mathrm{eV} \cdot \mathrm{s}^{-1}\left(\frac{100 \mu \mathrm{m}}{r_{p}}\right)^{2}\left(\frac{I_{e^{-}}}{1 \mu \mathrm{A}}\right) .
$$

This heating will be balanced by the cyclotron cooling of the positrons in the magnetic field, which occurs on a timescale of

$$
\tau_{r} \equiv \frac{9 m c^{3}}{8 e^{2} \Omega^{2}} \approx 4 \mathrm{~s}\left(\frac{10 \mathrm{kG}}{B}\right)^{2} .
$$

This leads to an equilibrium temperature,

$$
k T_{e q}=\tau_{c} \frac{d E}{d t}=0.81 \mathrm{eV}\left(\frac{10 \mathrm{kG}}{B}\right)^{2}\left(\frac{100 \mu \mathrm{m}}{r_{p}}\right)^{2}\left(\frac{I_{e^{-}}}{1 \mu \mathrm{A}}\right) .
$$

A possible source of additional heating is the beam-plasma instability. In this process, beam electrons interact with a longitudinal wave in the positron plasma. If the phase velocity of the wave is near the velocity of the electrons, the wave can gain energy from the beam. This results in a bunching of the beam, which in turn 
enhances the energy transfer. Wave growth saturates nonlinearly by heating the plasma particles.

The dispersion relation for electrostatic waves in a cold, unmagnetized plasma and a relativistic beam is [14],

$$
\varepsilon(\mathbf{k}, \omega)=0=1-\frac{\omega_{p}^{2}}{\omega^{2}}-\frac{\omega_{b}^{2}}{\gamma^{3}\left(\omega-c \mathbf{k} \cdot \beta_{b}\right)^{2}} .
$$

The subscripts $p$ and $b$ refer to the plasma and beam, respectively. All quantities are measured in the lab frame. In the beam term, two factors of $\gamma$ come from the relativistic Doppler shift of the wave in the beam frame, and one factor results from Lorentz contraction in the calculation of the density in $\omega_{b}$. This equation also applies in a magnetized system when all motion is in the direction of the field. When the system is not neutralized, there is also a frequency shift due to rotation of the particles in the electric field, but this shift is very small in the parameter regime of this experiment.

For the Bhabha experiment, $\omega_{b} \ll \omega_{p}$, and $\gamma^{3} » 1$, so the beam term in the 
dispersion relation is very small unless $\omega \cong \omega_{p}$ and $\omega / k=c$. For a plasma density of $10^{10} \mathrm{~cm}^{-3}$, the wavelength of the unstable mode is at least $30 \mathrm{~cm}$. The instability has at most one wavelength of growth before the beam particles exit the plasma, and should have a negligible effect on the system.

\subsubsection{Scattering Rate}

In competition with the temperature concerns is the need for a high data rate. The event rate is given by,

$$
R=1.87 \mathrm{~s}^{-1}\left(\frac{\sigma}{1 \mathrm{~b}}\right)\left(\frac{I_{e^{-}}}{1 \mu \mathrm{A}}\right)\left(\frac{n_{e^{+}} L}{3 \times 10^{11} \mathrm{e}^{+} / \mathrm{cm}^{2}}\right) \varepsilon_{e^{+} e^{-}}
$$

where $\sigma$ is the collision cross section and $\varepsilon_{e^{+} e^{-}}$is the efficiency in detecting both scattered particles. The cross section for Bhabha scattering at $2.3 \mathrm{MeV}$ is $\approx 50 \mathrm{mb}$. The detector geometry takes advantage of the fact that scattered particles remain pinned to magnetic field lines, resulting in a high detection efficiency of approximately 0.8 , giving a combined detection efficiency of $\varepsilon_{e^{+} e^{-}}=0.64$. Therefore, assuming $n_{e^{+}}=10^{10} \mathrm{~cm}^{-3}$ and $L=30 \mathrm{~cm}$, the 
incident electron current will have to be $\approx 10 \mu \mathrm{A}$ to achieve count rates close to $1 \mathrm{~s}^{-1}$.

Given this incident current, the radius of the plasma must be $\approx 500 \mu \mathrm{m}$ in order to maintain a positron temperature of $100 \mathrm{~K}$. This means that at least $2 \times 10^{9}$ electrons must be accumulated in order to achieve the desired density of $10^{10} \mathrm{e}^{+} / \mathrm{cm}^{3}$.

\subsubsection{Accumulation Rate}

The next concern in the design of the experiment is the rate at which positrons can be accumulated in the trap. The actual data rate in the experiment will be reduced from the value calculated in the previous sections by the duty factor of the positron trap.

A schematic of the basic accumulation scheme is shown in Fig. 1.2. Positron pulses from the linac are initially captured by time-of-flight (TOF). Between pulses, captured particles cool their axial energy by collisions, which transfer the energy into the transverse directions, where it is damped by cyclotron radiation. The positron-positron 


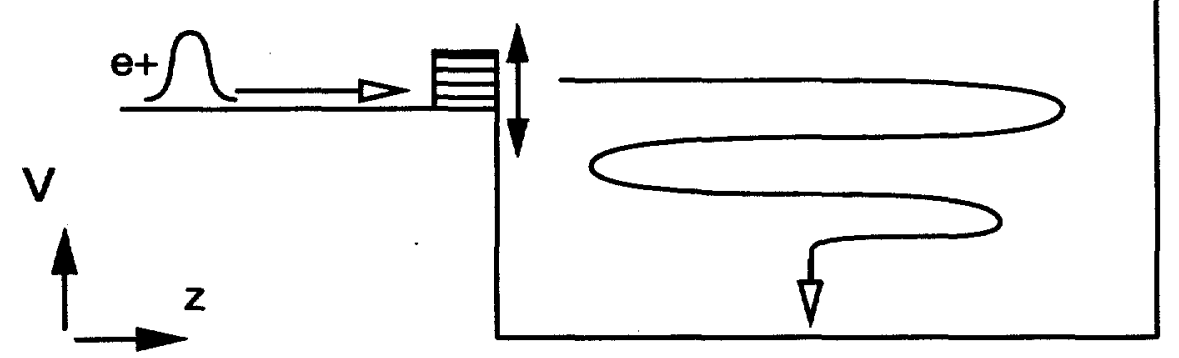

Figure 1.2 Positron accumulation schematic. Beam pulse is captured time-of-flight, then cools into the potential well.

collision frequency is,

$$
v_{c} \approx(3 \ln \Lambda) \mathrm{s}^{-1}\left(\frac{n}{10^{6} \mathrm{~cm}^{-3}}\right)\left(\frac{T}{1 \mathrm{eV}}\right)^{-3 / 2},
$$

where the Coulomb parameter $\ln \Lambda=\ln \left(\lambda_{D} / b_{\min }\right) \approx 15-20$.

For $t \ll v_{c}^{-1}$, accumulation follows the simple TOF formula,

$$
d N=N_{o} R_{\mathrm{inj}} d t-\frac{\Delta t}{t_{f}} R_{\mathrm{inj}} N d t
$$

where $N_{0}$ is the number of positrons in a pulse, $R_{\text {inj }}$ is the injection rate, $\Delta t$ is the time width of the injection gate, and $t_{f}$ is the roundtrip flight time in the trap. This assumes that the positrons disperse 
evenly through the trap in the time between pulses, and are not lost radially from the trap. The solution of this equation,

$$
N(t)=N_{o} \frac{t_{f}}{\Delta t}\left[1-\exp \left(-\frac{\Delta t}{t_{f}} R_{\mathrm{inj}} t\right)\right],
$$

asymptotically approaches the capture of $(t / \Delta t)$ pulses.

For $t>v_{c}^{-1}$, one must account for the increase in $t_{f}$ and $v_{c}$ as particles cool and the density increases, and the fact that particles cooled into the well are no longer lost when the injection gate opens. Additional improvement can be made when the well depth is ramped down slowly during injection instead of dropping the early positrons off a steep potential "cliff."

Figure 1.3 shows a numerical calculation of trap accumulation for the expected experimental parameters. Accumulation slows as it approaches the TOF limit. Efficient accumulation resumes when the trapped particles cool and the density is high enough that $v_{c}>R_{i n j}$ Injected particles then scatter into the potential well and are trapped 


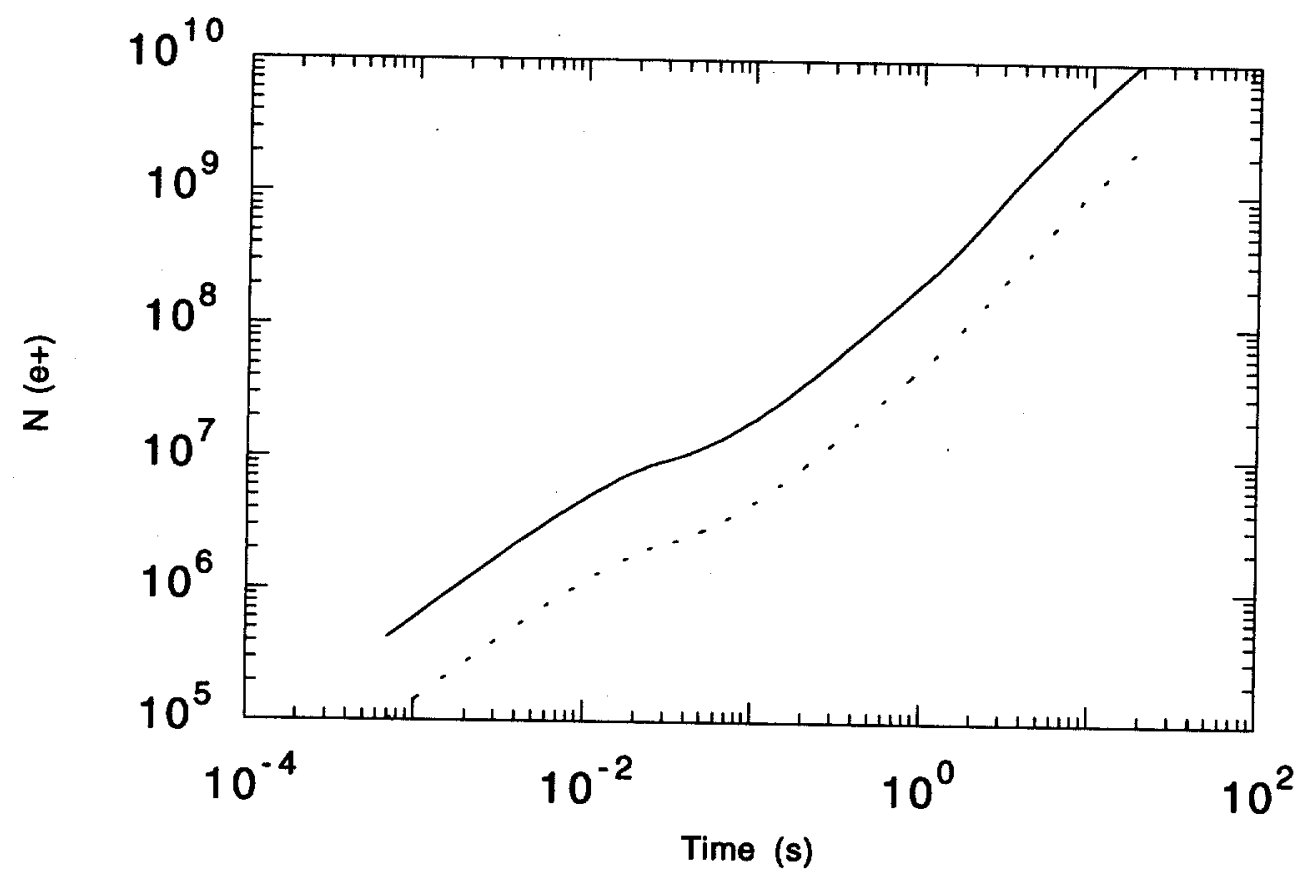

Figure 1.3 Calculated positron accumulation, showing density (-) and total number (--) of trapped positrons. $B=6 \mathrm{~T}, \mathrm{~L}=30 \mathrm{~cm}, \mathrm{~N}_{0}=1 \mathrm{e} 5, \mathrm{r}=0.05 \mathrm{~cm}$. Trap potential ramped to $-100 \mathrm{~V}$ over $1.2 \mathrm{~s}$.

before the next pulse is injected.

\subsection{Nonneutral Plasma Confinement}

The calculations in the previous section assume that trapped particles will not escape radially from the trap on the timescale of the accumulation. If $\approx 20 \mathrm{~s}$ are required to fill the trap, then the positron plasma should remain near the optimum density for $>200 \mathrm{~s}$ 


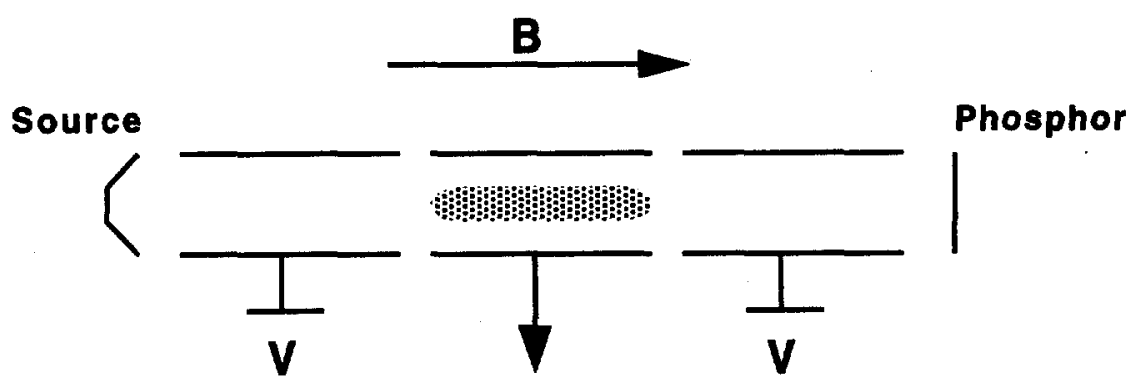

Figure 1.4 Cross-section schematic of a PenningMalmberg trap.

in order to achieve a real $1 \mathrm{~s}^{-1}$ data rate in the Bhabha scattering experiment. The confinement characteristics of nonneutral plasmas in Penning-Malmberg traps are thus crucial to the success of the experiment.

Nonneutral plasmas, because they contain only a single charge species, can be confined for long periods of time (typically $10^{2}-10^{3} \mathrm{~s}$ ) with static electric and magnetic fields [15]. A schematic crosssection of a basic nonneutral plasma trap is shown in Fig. 1.4. The trap is composed of a series of cylindrical electrodes providing an electrostatic well. The axial magnetic field provides confinement in the radial direction by limiting particle motion to small cyclotron orbits. The cyclotron radius of a positron or electron is 


$$
\rho=\frac{\mathrm{v}_{\perp}}{\Omega}=2.4 \mu \mathrm{m}\left(\frac{10 \mathrm{kG}}{B}\right)\left(\frac{T}{1 \mathrm{eV}}\right)^{1 / 2} .
$$

The basic dynamics of an infinitely long plasma column can be determined by assuming a uniform charge density out to a given radius, where the density drops off in a Debye length. This charge distribution has been shown to be a thermal equilibrium for both infinite and finite length plasmas $[16,17]$. It also produces a steadystate solution to Vlasov's equations $(\partial / \partial t \rightarrow 0)$ [18]. The motion that dominates the dynamics of these systems is bounce-averaged $E \times B$ drift, in which the particles behave like continuous lines of charge, moving perpendicular to both fields with a velocity,

$$
\mathbf{v}_{d}=c \frac{E \times B}{B^{2}}
$$

This is a valid treatment as long as the velocity of the cyclotron motion $v_{c}$, the axial "bounce" velocity $v_{b}$, and the drift velocity satisfy the relationship $v_{c} \gg v_{b} \gg v_{d}$.

The plasma itself generates a radial electric field, 


$$
E=\frac{\lambda e}{2} r
$$

where $\lambda$ is the density per unit length and $r$ is the radius inside the charge column. In an axial magnetic field, $\mathbf{v}_{d}$ is in the angular direction and is proportional to the radius. This causes the entire column to rotate with a uniform frequency,

$$
\omega_{\theta}=\frac{\mathbf{v}_{d}}{r}=\frac{c \lambda e}{2 B} \text {. }
$$

The above treatment does not address the question of stability of the plasma equilibrium. A simple, heuristic argument for stable confinement can be made from conservation of angular momentum [19]. The canonical angular momentum of the system is given by,

$$
P_{\theta}=\sum_{j}\left[m v_{\theta j} r_{j}-(e / c) A_{\theta}\left(r_{j}\right) r_{j}\right]
$$

where $A_{\theta}(r)$ is the vector potential. If we assume a uniform axial magnetic field, $A_{\theta}(r)=B r / 2$, where $B$ is sufficiently large that the field term dominates the angular momentum, then

$$
P_{\theta} \propto(-e B / 2 c) \sum_{j} r_{j}^{2}
$$


Conservation of angular momentum in the electrostatic interactions between particles then provides a constraint on the radial positions of the particles, and only a small fraction can reach the electrode wall at a radius much larger than the initial plasma radius. The limit on particle loss is $\Delta N / N \leq(a / R)^{2}$, where $N$ is the total number of particles, $a$ is the initial plasma radius, and $R$ is the wall radius. By this argument, deconfinement of the plasma must be caused by processes which do not conserve the angular momentum of the system. Possible mechanisms include collisions with neutral atoms, asymmetric field errors, and finite wall resistance. In the Bhabha experiment, interaction between the positron plasma and the electron beam could also increase transport.

This argument ignores the kinetic angular momentum of the trapped particles, and also ignores the diamagnetic field produced by the motions of the particles. Similarly, the Vlasov equation treatment mentioned above is not strictly self-consistent because it also ignores the diamagnetic field. This is valid as long as the particles are nonrelativistic, but in principle the electric field of the plasma could do work on escaping particles such that they achieve high velocity. A 
more thorough confinement analysis has been performed which includes the terms due to fast particles [20] and calculates a slightly modified constraint expression; however, the modifications are insignificant for current laboratory plasma parameters. Most importantly, deconfinement processes are still limited to those which transfer angular momentum to the particle and field system.

A detailed discussion of several possible deconfinement mechanisms can be found in Appendix A. The dominant working model, when the trap was designed, was single particle bounce-resonance, in which the particle bounce frequency in the trap $\omega_{b}$ is a harmonic of the bulk plasma rotation $\omega_{r}$, causing particles to interact resonantly with field errors [21]. This phenomenon has been studied extensively in the context of magnetic mirror traps [22].

Experiments performed at UC San Diego (UCSD) find that trap confinement time scales as $\tau_{m}=0.32(B / L)^{2}$, where $\tau_{m}$ is the time for the central density to reach half of its initial value [23]. This represents an improvement of a factor of 20 over previous 
experiments, but with the same quadratic scaling [24]. The increase is attributed to improvements in alignment and uniformity of the fields. The bounce resonance model then suggests a density dependence of $n^{-2}$, though the $B / L$ scaling is determined from experiments with a nearly constant density of $\approx 1.2 \times 10^{7} \mathrm{~cm}^{-3}$.

Observations in similar traps qualitatively support the inverse density scaling, with an exponent for the density in the range of -1 to $-2[25,26]$, though no systematic study has been performed.

\subsection{Present Work}

This dissertation reports on the development of a positron trap at LLNL, and a comparison of the trap's electron confinement properties with similar devices in use at the University of California, San Diego (UCSD). Using electrons from a source similar to those used in the other devices, it exhibits comparable confinement times. Time-offlight accumulation of positrons from the accelerator-based positron source shows rapid deconfinement of the positrons, inconsistent with the long electron lifetimes. Several possible deconfinement 
mechanisms have been explored, including annihilation on background gases, injection heating, rf noise from the accelerator, magnet curvature, and stray fields.

Experiments with pulsed electrons demonstrate that there are several complications to pulsed injection. Fast switching of potentials can lead to substantial heating of the injected particles. Even a few electron volts of heating enhances the positron annihilation rate on background gases by several orders of magnitude. In addition, the confinement for small numbers of particles, such as in the early stages of accumulation, is much lower than predicted by the empirical lifetime scalings of high-density plasmas. Short electrodes, with axial dimensions comparable to radial extent, are required for improved confinement. The electrode structure has been redesigned to allow positron accumulation in several small electrodes.

A more quantitative study has been made of the confinement scaling properties of the trap with electrons. The results are largely inconsistent with the accepted scalings. Qualitatively, confinement does improve with shorter length and higher magnetic field, but the 
predicted quadratic dependence is not seen. In complete contrast to previous experiments, the confinement in this device improves with increased density. This singular inconsistency is enough to account for the difficulty in holding the first few, relatively diffuse pulses of positrons.

The effort for this thesis has revolved around development of the trap instrumentation and understanding the electron and positron confinement properties in the new parameter regime of this device. Chapter 2 gives a detailed description of the experimental apparatus, and a summary of its basic operation for electron and positron trapping. Chapter 3 reviews the efforts to accumulate positrons and discusses the unique issues associated with positron confinement and pulsed injection. Systematic confinement studies with electrons are presented in Chapter 4. Chapter 5 provides a summary of the results and a discussion of possible future improvements to the trap for future nonneutral plasma studies and positron accumulation. The various candidates for plasma deconfinement and the confinement characteristics of other electron and positron traps are discussed in Appendix A. Positron interactions with residual gas are discussed in 
Appendix B, with particular emphasis on the energy dependence of positronium formation. Appendix C presents numerical ray-tracing calculations of single-particle transport due to field errors. 


\section{Experimental Apparatus}

\subsection{Overview of the LLNL Nonneutral Plasma Trap}

The apparatus developed at LLNL is designed for the accumulation of high density positron and electron plasmas. An intense pulsed positron beam is provided by an electron linac. A continuous or pulsed beam of electrons is produced with a thermionic cathode. Particles are captured using a Penning-Malmberg trap in a largevolume, high-field superconducting solenoid.

\subsection{Positron Source}

The positron trap makes use of the LLNL High-Intensity Positron

Source [27]. The system is capable of producing over $10^{6}$ moderated positrons in $20 \mathrm{~ns}$ pulses at a repetition rate of up to $1440 \mathrm{~Hz}$.

Positrons can be transported at energies of $10-7000 \mathrm{eV}$. The positron production is driven by a $100 \mathrm{MeV}$ electron linear accelerator (linac). Positrons are transported from the production area to the trap 


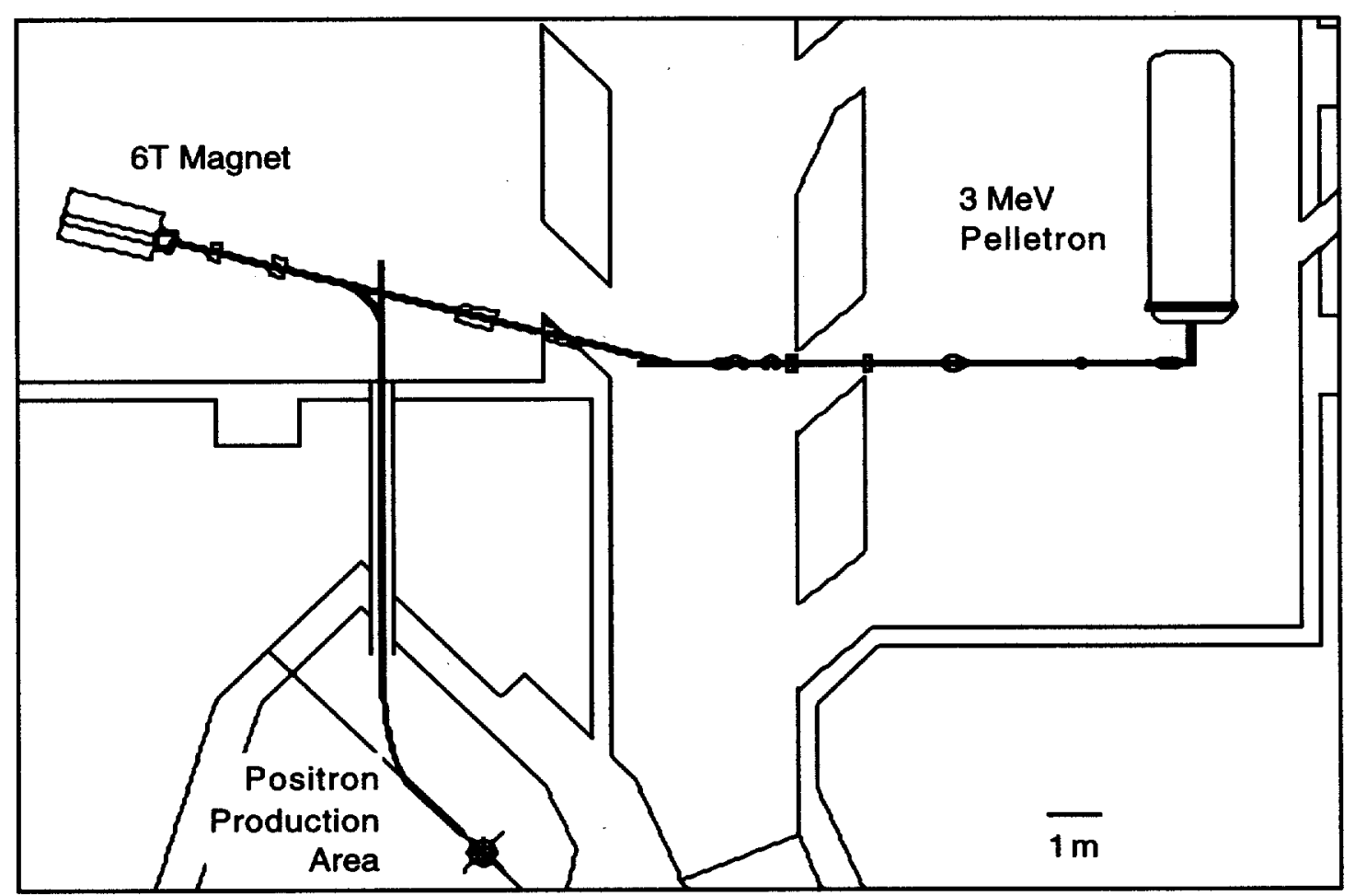

Figure 2.1 Diagram of the LLNL Positron Facility.

apparatus along a magnetic guide field. Figure 2.1. shows a plan view of the positron production and transport system. Also shown is the $3 \mathrm{MeV}$ electrostatic accelerator intended for use in the Bhabha scattering experiment.

The accelerator-based system has significant potential advantages over the radioactive source based systems currently in use elsewhere. The primary advantage is the higher beam intensity. In the short-pulse mode used for trapping, the accelerator produces 
over $10^{9}$ moderated positrons per second. A radioactive source based system would have to use a dangerously large $6.8 \mathrm{Ci}$ source to generate moderated positrons at a comparable rate, assuming an excellent moderator efficiency of $4 \times 10^{-3}$.

The second advantage is the pulsed nature of the accelerator generated beam. Radioactive sources are inherently continuous, requiring complex and inefficient systems to accumulate the positrons. A short pulse of positrons can be captured with nearly $100 \%$ efficiency by time-of-flight (TOF) trapping.

\subsubsection{Accelerator}

The linac is a radio frequency electron accelerator. Electrons from a cathode are bunched and then accelerated through five rf cavities, receiving approximately $20 \mathrm{MeV}$ in each stage. The electron gun can supply pulses $20 \mathrm{~ns}$ to $2.8 \mu \mathrm{s}$ in length. In short pulse mode, the repetition rate can be as high as $1440 \mathrm{~Hz}$, while in long pulse mode the rate is limited to $360 \mathrm{~Hz}$. Peak electron current can reach over 5 A for short pulses, and $1 \mathrm{~A}$ for longer pulses. The long pulse mode 
can provide larger average positron production rates due to its larger duty cycle, but the short pulse mode was used in these experiments to facilitate TOF trapping.

\subsubsection{Positron Converter and Moderator}

The converter and moderator assembly is referred to collectively as the "positron front end." A schematic is shown in Figure 2.2. The electron beam exits the accelerator beamline into the air through two $50 \mu \mathrm{m}$ thick aluminum windows and strikes the positron converter target. The aluminum windows are water cooled around their circumference, and the space between the windows is pressurized to $69 \mathrm{kPa}(10 \mathrm{psi})$ to enhance convective cooling. The positron converter is $1 \mathrm{~cm}$ thick tungsten surrounded by water cooled copper. The $100 \mathrm{MeV}$ electrons decelerate rapidly in the tungsten, generating an intense beam of bremstrahlung gamma rays. The high energy photons convert to positron-electron pairs in the high nuclear fields of the tungsten. These new particles in turn decelerate and radiate, creating more pairs. The result is a cascade of positrons, electrons, and photons through the converter. 


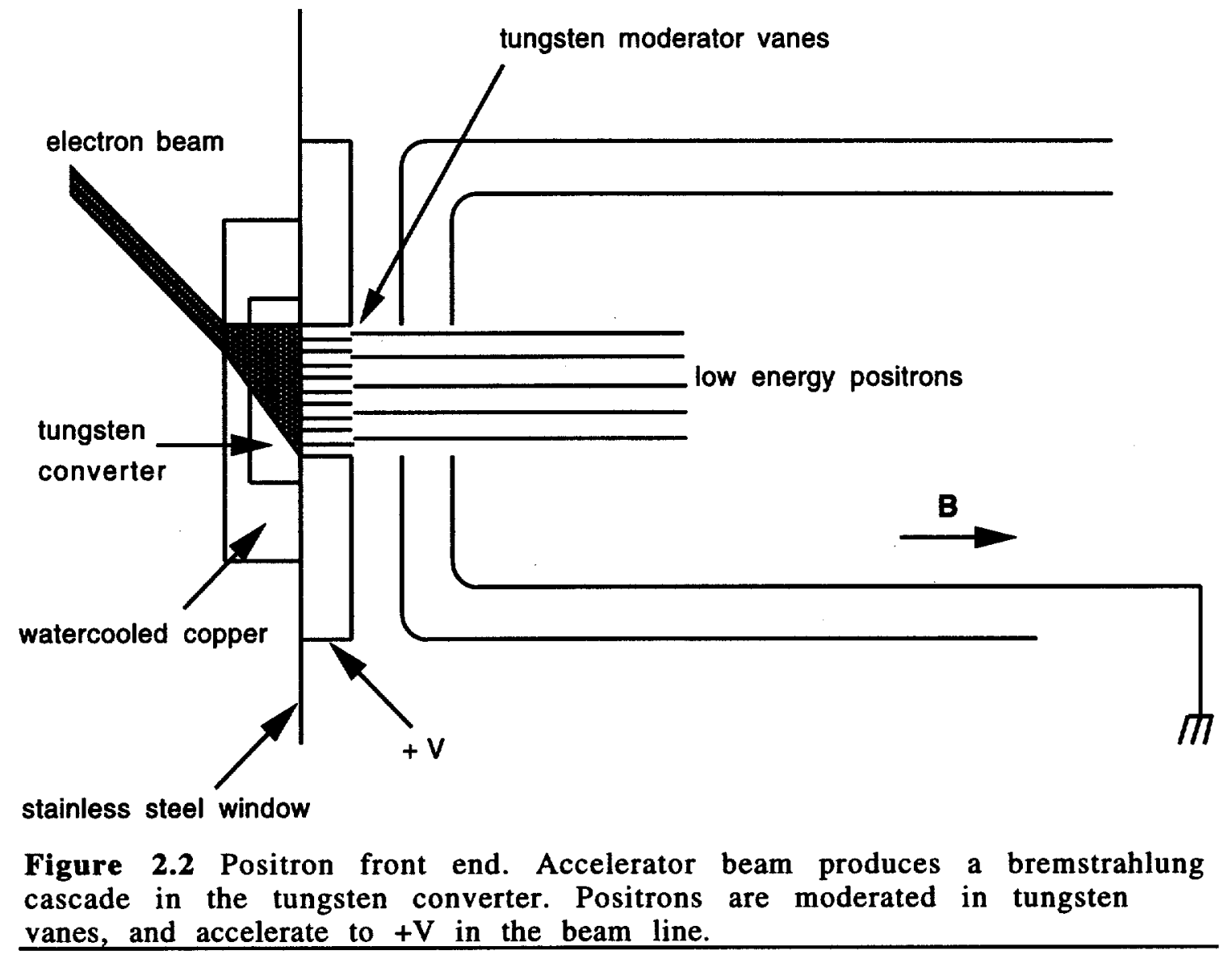

The beam then enters the positron transport vacuum system through a $25 \mu \mathrm{m}$ stainless steel window. The particles pass through a set of $25 \mu \mathrm{m}$ horizontal tungsten moderator vanes. Some of the positrons enter the tungsten and thermalize. Tungsten is used as a moderator because it has a negative surface work function for positrons.

Thermal positrons diffusing to the surface of a vane are ejected with the work function energy of approximately $3 \mathrm{eV}$. The energy spread is nominally thermal, though inelastic scattering from surface 
electrons, phonons, and impurities increases the spread to $1-2 \mathrm{eV}$ [28]. Because the foils are oriented horizontally, elastic scattering and microscopic surface roughness can also increase energy spread by widening the emission cone. In the accelerator environment, the dominant source of beam energy spreading is the linac electron beam. The intense electron pulse causes a fast oscillation of the bias voltage that can be as high as $25 \mathrm{~V}$ at peak electron current of $5 \mathrm{~A}$.

\subsubsection{Transport beamline}

The front end is electrically isolated from the rest of the positron transport system. A bias of $10-7000 \mathrm{~V}$ is applied to accelerate the positrons into the beamline. Beam confinement and steering is provided by magnetic guide fields. Solenoids produce an axial field of approximately $100 \mathrm{G}$. Small coils arranged in Helmholtz pairs oriented perpendicular to the beamline generate steering fields.

The positron front end is situated inside three magnet coils. From there, positrons enter a curved section configured as a continuous toroid with approximately $4 \mathrm{~m}$ major radius that directs the beam out of the production area. Long steering coils compensate for beam 
drift caused by the curvature. The straight section passing through the wall from the production area into the experimental area is divided into two independent solenoid and steering coil sections. In the experimental area, a series of 20 pancake coils guide the beam around a second curve with radius of $2.2 \mathrm{~m}$ and into the trap magnet. An insertable microchannel plate (MCP) and phosphor assembly for viewing the positron beam are placed in the straight section before entry into the high fields of the trap. See Section 2.5.1 for a detailed description of MCP operation.

Optimum transport for this experiment requires a smooth magnetic field so that the positrons will adiabatically follow magnetic field lines. The calculated optimum field profile along the transport axis is shown in Fig. 2.3. The smooth field configuration does not always produce the most intense incident positron beam, but does appear to maximize transmission into the trap. This suggests that non-adiabatic changes in the magnetic field can convert some of the longitudinal energy of the positrons into transverse energy. When the particles pass from the transport field into the large trap field, excess transverse energy can result in magnetic mirroring. 


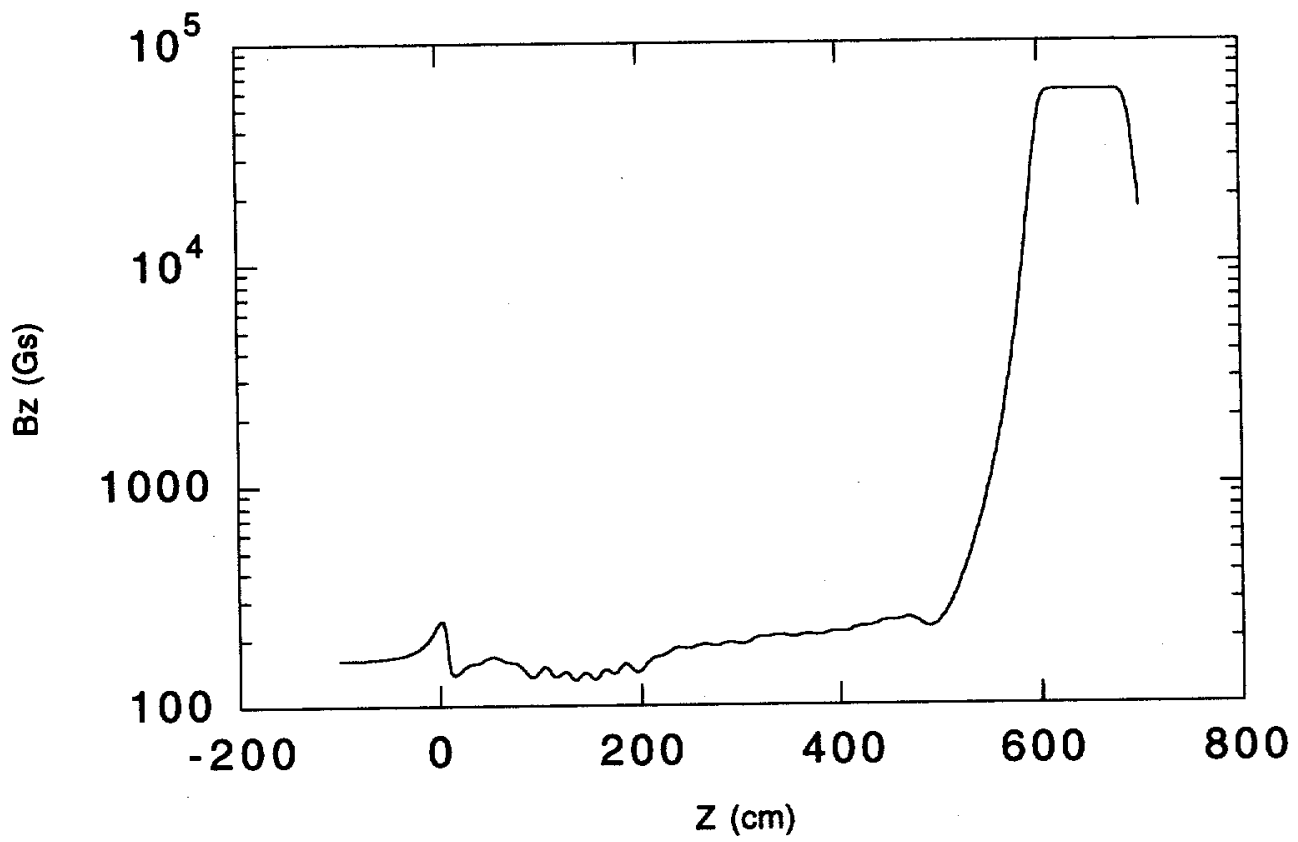

Figure 2.3 Calculated axial magnetic field in positron transport beamline. $Z$ is measured along beam path. Origin is at the wall of the experimental area.

Particles travelling adiabatically in an increasing magnetic field can be reflected if the pitch angle of their motion is too high. The requirement for adiabaticity is that spatial field variations are small over one cyclotron orbit,

$$
\left|\frac{\mathbf{v} \cdot \nabla \mathbf{B}}{\Omega \mathbf{B}}\right|<1
$$

where $\Omega$ is the cyclotron frequency. The magnetic moment associated with a particle's cyclotron motion, 


$$
\mu=\frac{W_{\perp}}{B}
$$

where $W_{\perp}$ is the transverse energy, is approximately conserved for adiabatic motion. To avoid reflection, $W_{\perp}<W$ at the final magnetic field strength. This gives a limit on the initial transverse energy,

$$
\frac{W_{\perp i}}{W}<\frac{B_{i}}{B_{f}}
$$

For $B_{i}=200 \mathrm{G}$ and $B_{f}=30 \mathrm{kG}$, the maximum transverse energy is then only

$$
W_{\perp}^{\max }=6.7 \times 10^{-3} W
$$

requiring a pitch angle less than $4.7^{\circ}$. For $B_{f}=60 \mathrm{kG}$, the acceptance angle shrinks to $3.3^{\circ}$.

Due to the mirroring effect, high-energy transmission through the magnet at $30 \mathrm{kG}$ is typically $40-50 \%$, occasionally reaching $60 \%$. At transport energies $<200 \mathrm{~V}$, careful tuning has achieved transmission over $80 \%$. This suggests that nonadiabatic transitions in the beamline may be causing energy spreading in the high-energy beams. The 
sharp field transition seen at $z=0$ in Fig. 2.3 is a likely candidate, and transmission is found to be highly sensitive to the field in that region.

The longitudinal energy profile of the positron beam is measured by placing a screen barrier in front of the MCP. The MCP then measures the total charge transmitted over the barrier. Figure 2.4 shows the integrated energy distribution for a $4.9 \mathrm{keV}$ beam. For the data in Fig. 2.5, the $50 \mathrm{~V}$ beam is analyzed using the screen and an electrode inside the $30 \mathrm{kG}$ trap region. The lack of "spin-up" as the beam enters the high field is consistent with the high transmission at low energy. The transmitted distribution for higher-energy beams could not be measured because the trap electrodes were not designed for high voltage.

\subsubsection{Remoderator}

When the short timing characteristics of the positron pulse must be preserved in transport, the beam is operated at an energy of 5-7 keV. Fast switching of electrodes at such high voltages would be difficult, so a $1000 \AA$ tungsten remoderator foil is inserted into the 


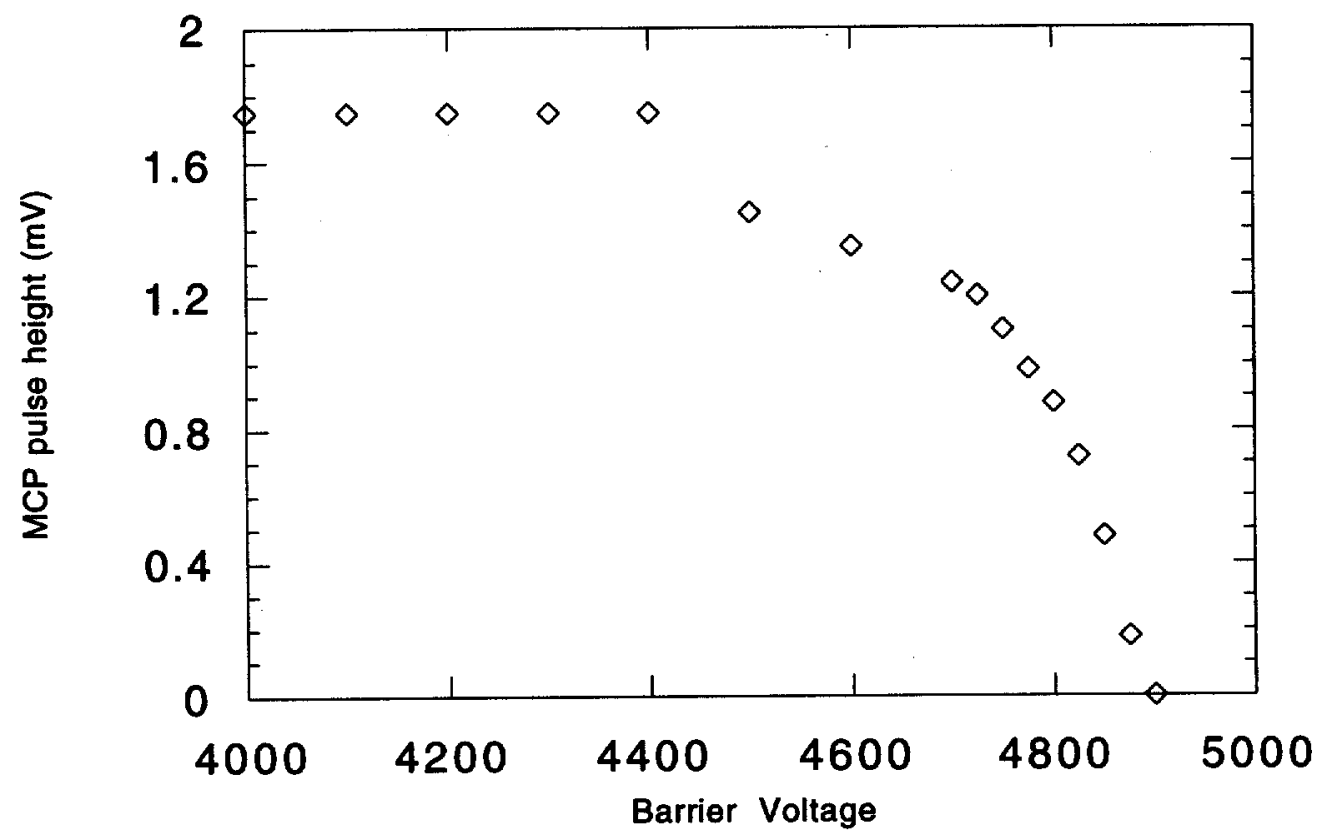

Figure 2.4 Integrated energy distribution of $4.9 \mathrm{keV}$ positron beam. For fast pulses, the height is proportional to $\mathrm{N}_{\mathrm{e}^{+}}$transmitted over the barrier.

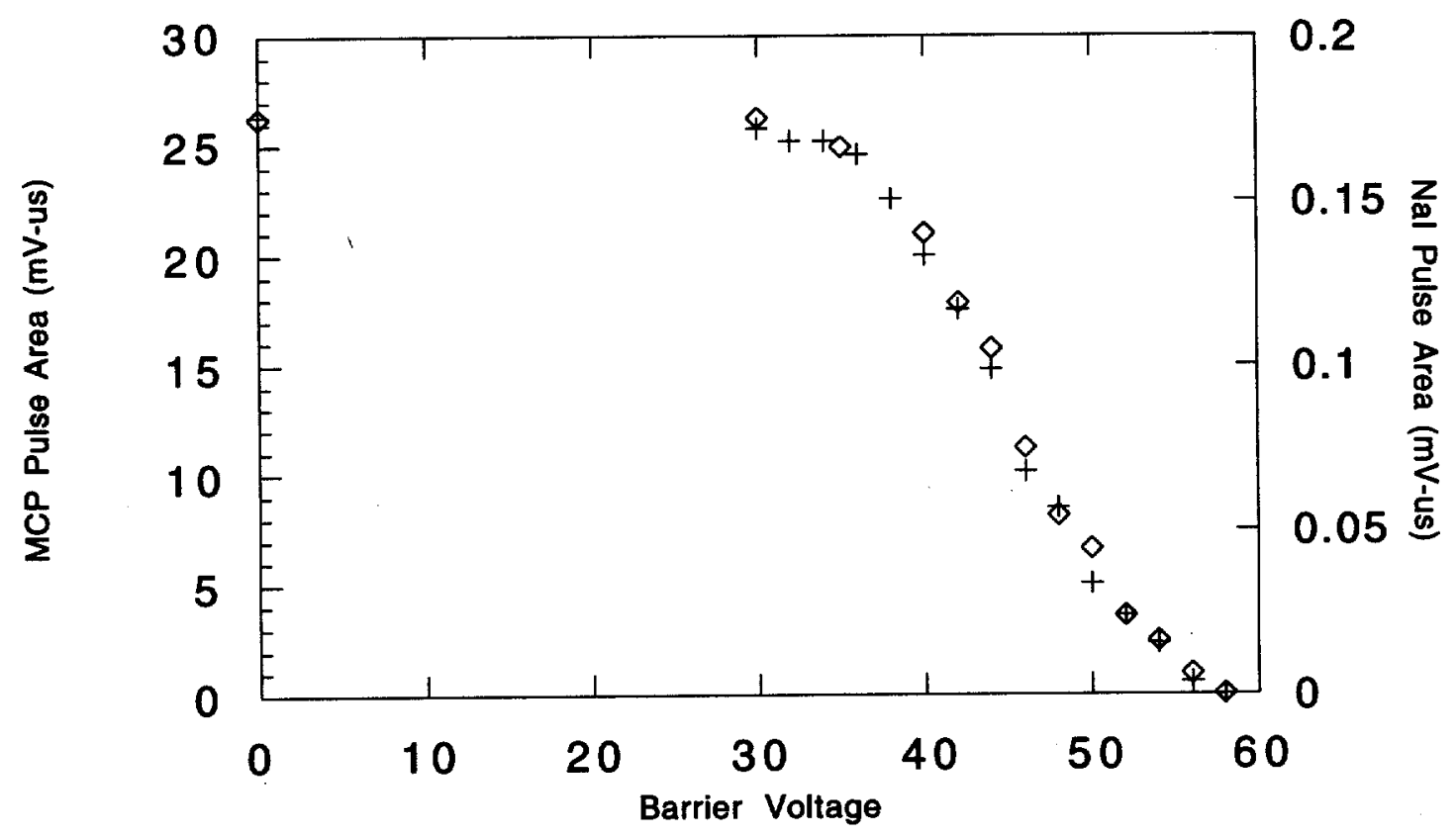

Figure 2.5 Integrated energy distribution for $50 \mathrm{eV}$ beam. Pulse area measured from the MCP before the magnet $(0)$ and the annihilation signal in a NaI crystal after the magnet $(t)$. 
beam path at the front end of the trap. The positrons thermalize in the foil and enter the trap with only a few eV. There is no detectable increase in the time width of the pulse in this process, and the large transverse positron energy caused by entering the high magnetic field is reduced to a thermal spread; however, most of the positron intensity is lost to annihilation in the foil.

Remoderator efficiency is typically only $4-5 \%$. This could perhaps be improved by a factor of 2-4 with in-situ annealing of the foil. Part of the inefficiency is also caused by foil nonuniformity, allowing some high-energy positrons to pass through the thin areas with minimal energy loss. A large longitudinal energy spread in the beam, due to spin-up in the magnetic field, can also reduce efficiency because the thickness of the foil is optimized for $5-7 \mathrm{keV}$ transport. As a result of the mirroring and foil inefficiency, only $1-2 \times 10^{4}$ moderated positrons are typically injected into the trap with each pulse, more than a factor of 5 below design parameters. This had a clear impact on accumulation, as will be shown in Chapter 3. 


\subsection{Electron source}

In most nonneutral plasma devices, electrons are supplied by a spiral filament immediately adjacent to the first trap electrode. The spiral is designed so that the potential drop across the radius of the cathode closely matches the potential profile of the ideal uniform density plasma [29]:

$$
\varphi(r) \propto V_{b}+V_{f} r^{2} / R_{f}^{2}
$$

$V_{b}$ is the bias voltage, $V_{f}$ is the voltage drop across the filament, and $R_{f}$ is the radius of the spiral. In early testing of the trap performance, a spiral filament designed for high density experiments was used [30]. This filament consisted of $50 \mu \mathrm{m}$ diameter tungsten wire, stitched through holes in a $250 \mu \mathrm{m}$ thick ceramic plate. The thinner wire creates a larger voltage drop, generating a higher density plasma. The ceramic plate provides support for the thin wire and allows precision construction of the spiral.

In the long term, the standard filament placement was not feasible for the LLNL system, since the filament had to be easily removable 
for positron injection, and the positron remoderation foil was placed at the front of the trap. For testing purposes, the spiral was initially placed in an immobile fixture in the fringe field. The stitched spiral lead to problems with reproducibility, because the temperature of individual stitches was highly sensitive to the amount of thermal contact with the ceramic. This irregularity is noted in Ref. 30, but was minimized by long warm-up times. In the LLNL trap, transport through the large field gradient apparently enhanced problems due to spatial inhomogeneities, leading to unstable injection. In this configuration, the potential-matching filament is not clearly advantageous even with a uniform temperature. Field variation across the radius of the spiral may cause a radial dependence in the injection efficiency, complicating the relationship between $V(r)$ at the filament and the space charge potential in the trap.

The electron beam in the current system is provided by an indirectly-heated boron cathode, designed for the NEC $3 \mathrm{MeV}$ Pelletron. The cathode is placed in a close approximation to a standard Pierce gun electrode configuration [31], as shown in Fig. 2.6. The angle of the electrodes compensates for the repulsive space 


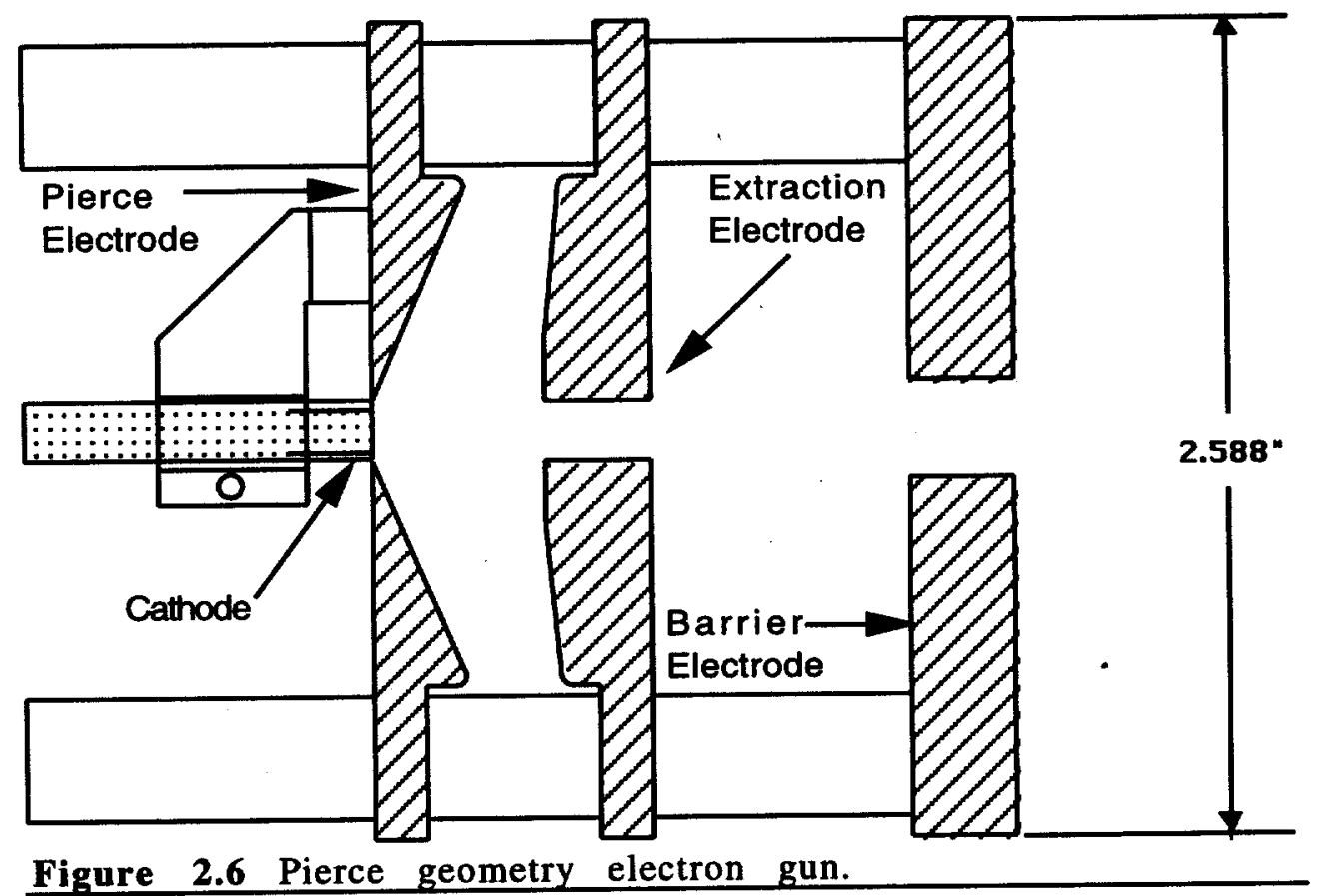

charge potential of the beam so that the resulting field lines, and therefore the beam, are straight. It is unclear whether this design is actually necessary when the magnetic field already provides a strong focusing, and the extraction voltage, typically $\approx 10 \mathrm{~V}$, is not high enough to generate a space-charge limited beam. The third electrode provides a barrier potential for energy analysis of the beam, or additional field shaping and acceleration. The whole assembly is situated on a rotatable mount for easy removal during positron injection.

Beam energy is varied by adjusting the bias on the cathode, and a 


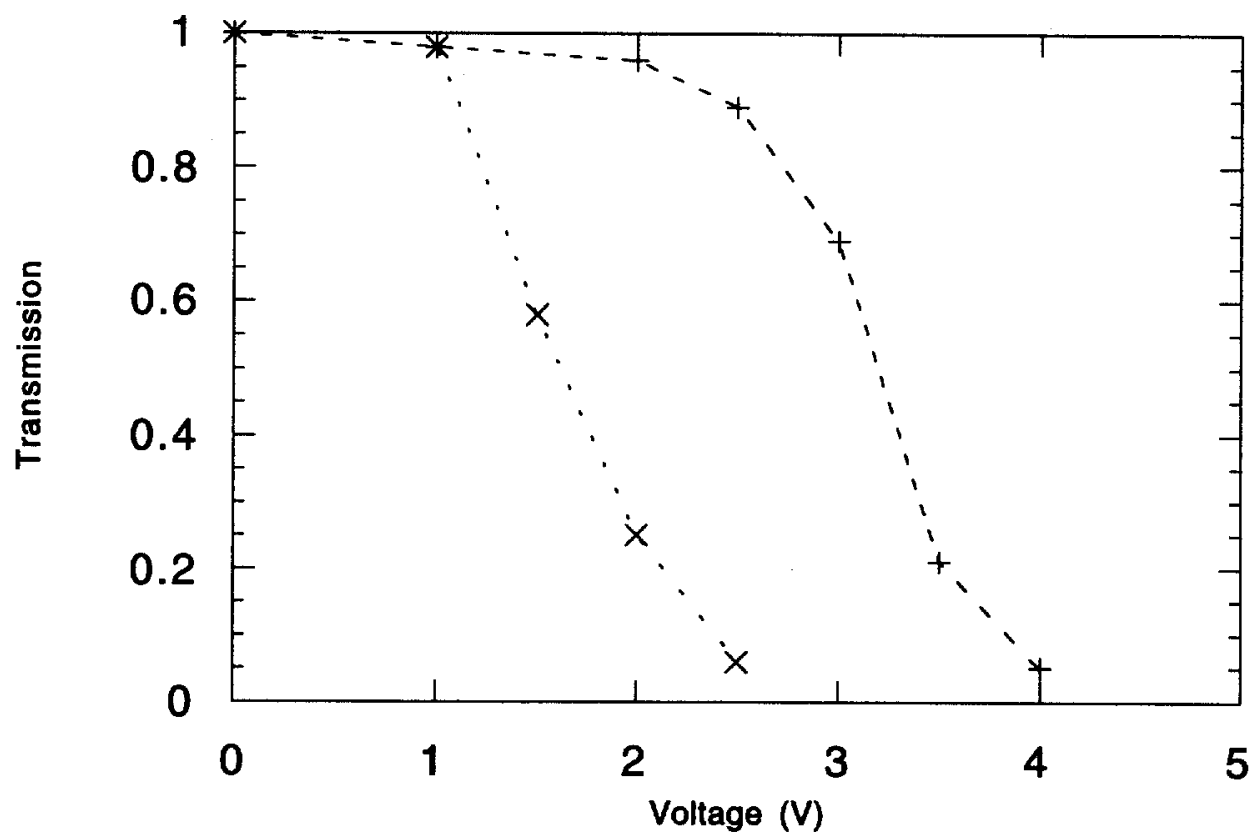

Figure 2.7 Electron spin-up. Integrated longitudinal energy distribution in the low $(+)$ and high $(x)$ magnetic field regions.

short pulse of electrons $(<1 \mu s)$ is produced by pulsing the cathode bias. The longitudinal energy distribution of the beam can be measured in the fringe field using the third electrode, and in the high field with a trap electrode as the barrier. The effect of spin-up as the electrons enter the high field can clearly be seen in Figure 2.7.

\subsection{Penning-Malmberg Trap}

In order to achieve long confinement times, the electrodes and solenoid magnet in the LLNL trap were manufactured to very high 


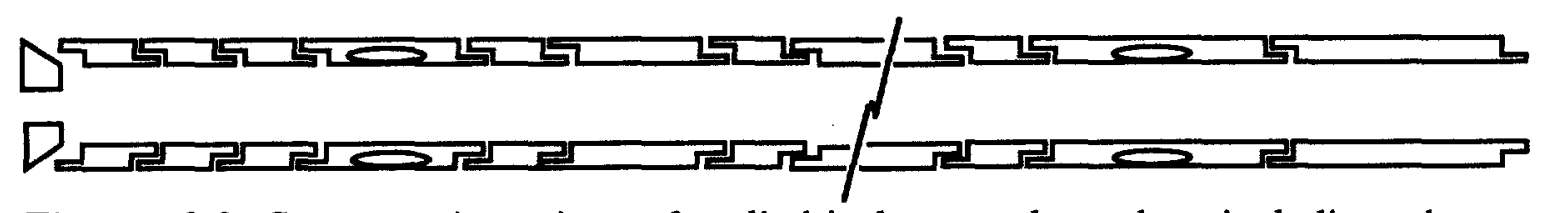

Figure 2.8 Cross section view of cylindrical trap electrodes, including short electrode from remoderator foil assembly. Six pairs of $2 \mathrm{~cm}$ and $4 \mathrm{~cm}$ electrodes are not shown. Ovals indicate sectored electrodes.

tolerances in order to minimize field errors. Most of the construction is of copper, aluminum, and other non-ferrous materials to limit stray magnetic fields. Low permeability stainless steel was used when low thermal conductivity was needed, and arranged symmetrically when possible.

\subsubsection{Electrodes}

The cylindrical electrodes are composed of oxygen-free highconductivity (OFHC) copper, plated with gold on the interior surfaces to prevent the formation of dielectric oxides. A side cross-section of the electrode configuration is shown in Fig. 2.8. Electrodes have lengths of 2,4 , and $6 \mathrm{~cm}$. Alumina spacers provide insulation between electrodes. The inner and outer diameters of the cylinders are concentric to within $10 \mu \mathrm{m}$ and vary by less than $2.5 \mu \mathrm{m}$ between electrodes. When assembled at room temperature, the internal trap surface is aligned to within $10 \mu \mathrm{m}$ over a $76 \mathrm{~cm}$ length. Some of the 4 


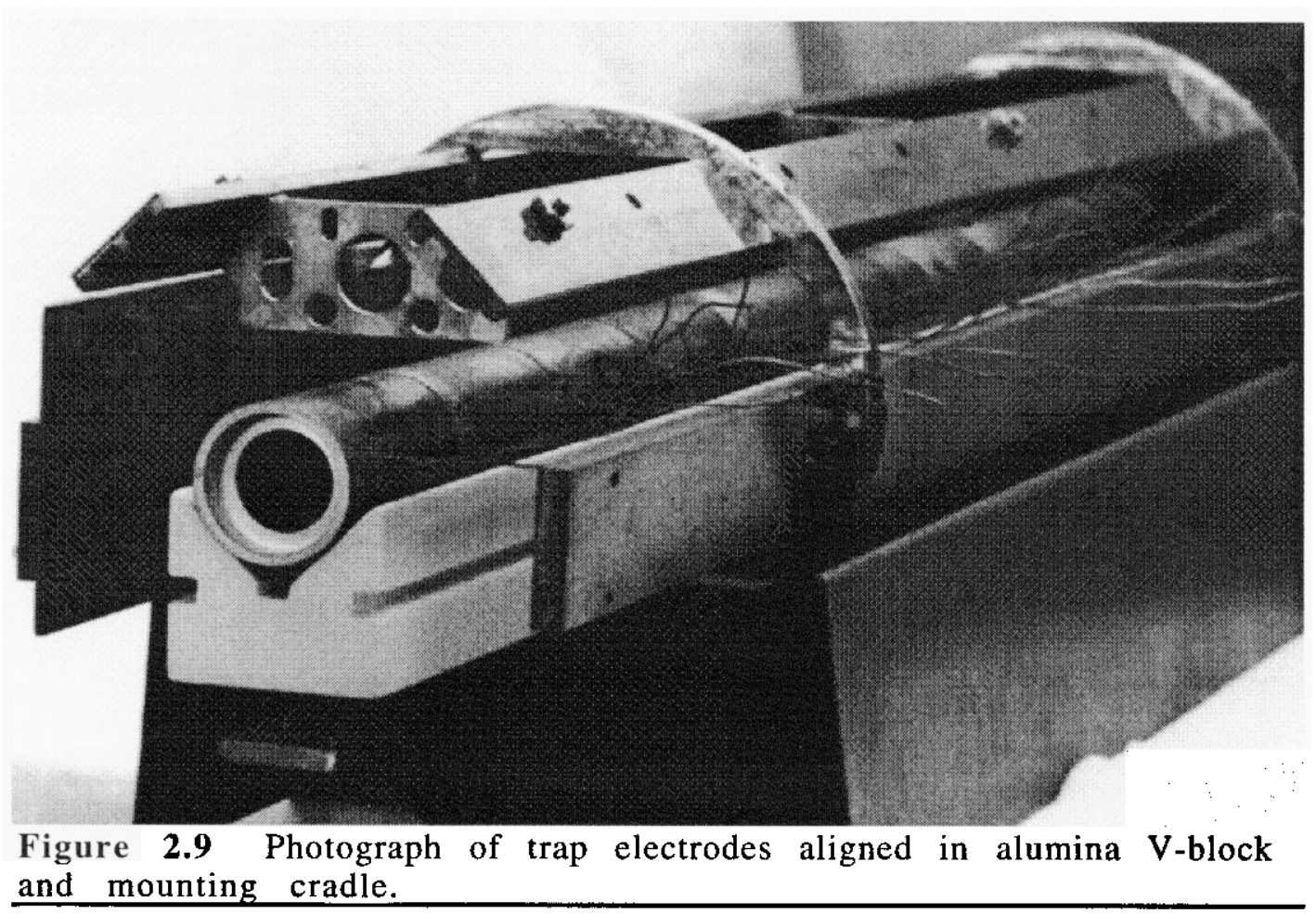

and $6 \mathrm{~cm}$ electrodes contain two isolated, oval "sectors" opposite one another. These can provide signals of off-axis motion of trapped particles, and can be used to drive the motion as well. The uses of sectored electrodes are discussed in more detail in Section 2.5.3.

The electrodes are aligned on an alumina V-block. The surface of the block is flat to within $5 \mu \mathrm{m}$ over the full $76 \mathrm{~cm}$ length. Alumina was chosen for its machinability, electrical insulation, and high thermal conductivity. The electrodes and mounting block are all placed in a cradle system, as shown in Fig 2.9, for placement inside the magnet 
bore. Cam-locking clamps are used to fix the cradle securely in place. Electrical connection to the electrodes is provided by small Kaptoninsulated coaxial cables of silver-plated copper. The 30-gauge (250 $\mu \mathrm{m}$ diameter) central conductor minimizes thermal conductance for efficient cryogenic operation.

\subsubsection{Magnet}

The trap is situated in the bore of a superconducting electromagnet. The magnet is composed of one central coil and two end coils, and is capable of generating a field over $60 \mathrm{kG}$ in a large volume. The field profile has been measured using a nuclear magnetic resonance (NMR) probe capable of measuring milligauss fields. The field strength, shown in Figs. 2.10 and 2.11 , varies by less than $0.1 \%$ axially over the length of the trap, and $\sim 20 \mathrm{ppm}$ peak-to-peak azimuthally at the electrode radius. The slight axial asymmetry is consistent with the displacement of the center coil by $0.13 \mathrm{~mm}$. The axial variation can be accounted for by a $1 \mathrm{~mm}$ horizontal offset of one end of the magnet, forming the solenoid into a section of a torus with a major radius of approximately $1 \mathrm{~km}$. 


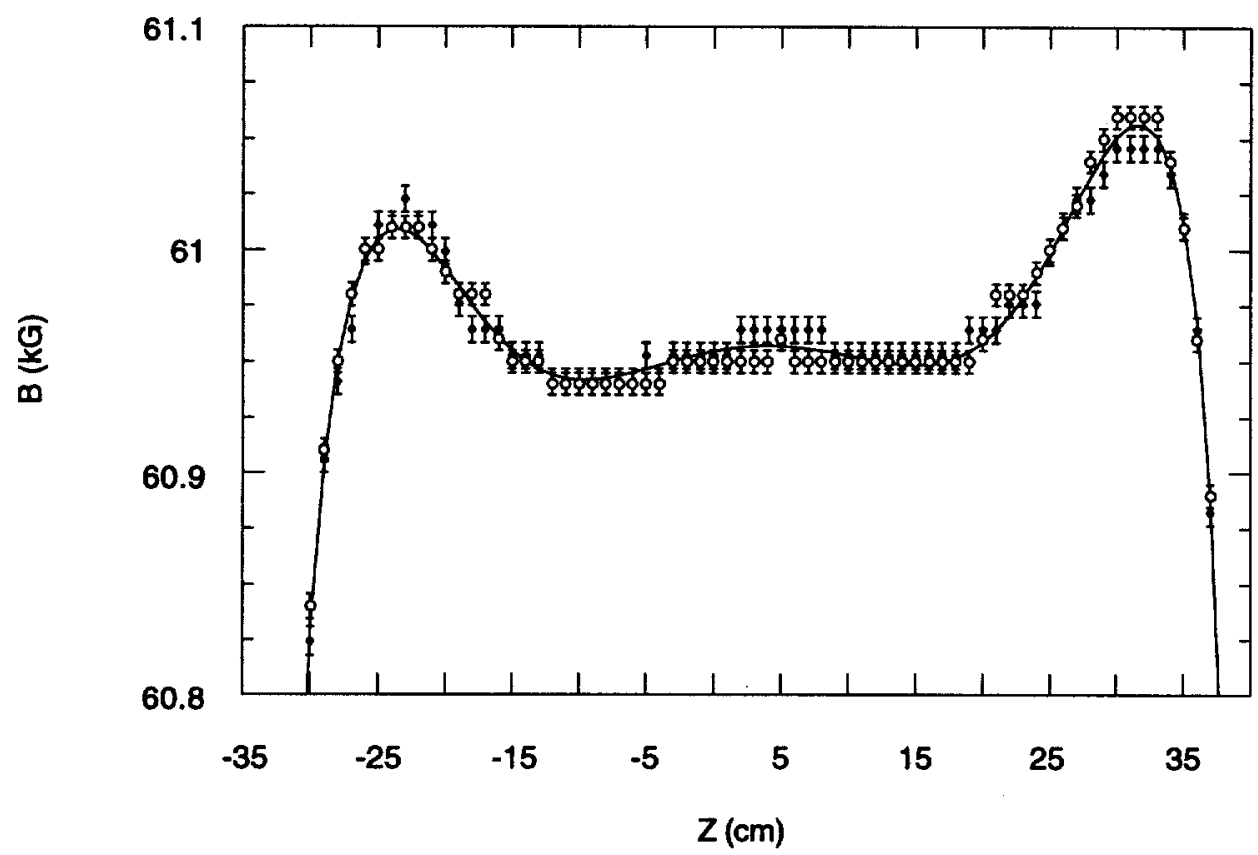

Figure 2.10 Total magnetic field strength along solenoid axis. Solid line is a fit allowing coil contraction during cooling and an offset of the central coil.

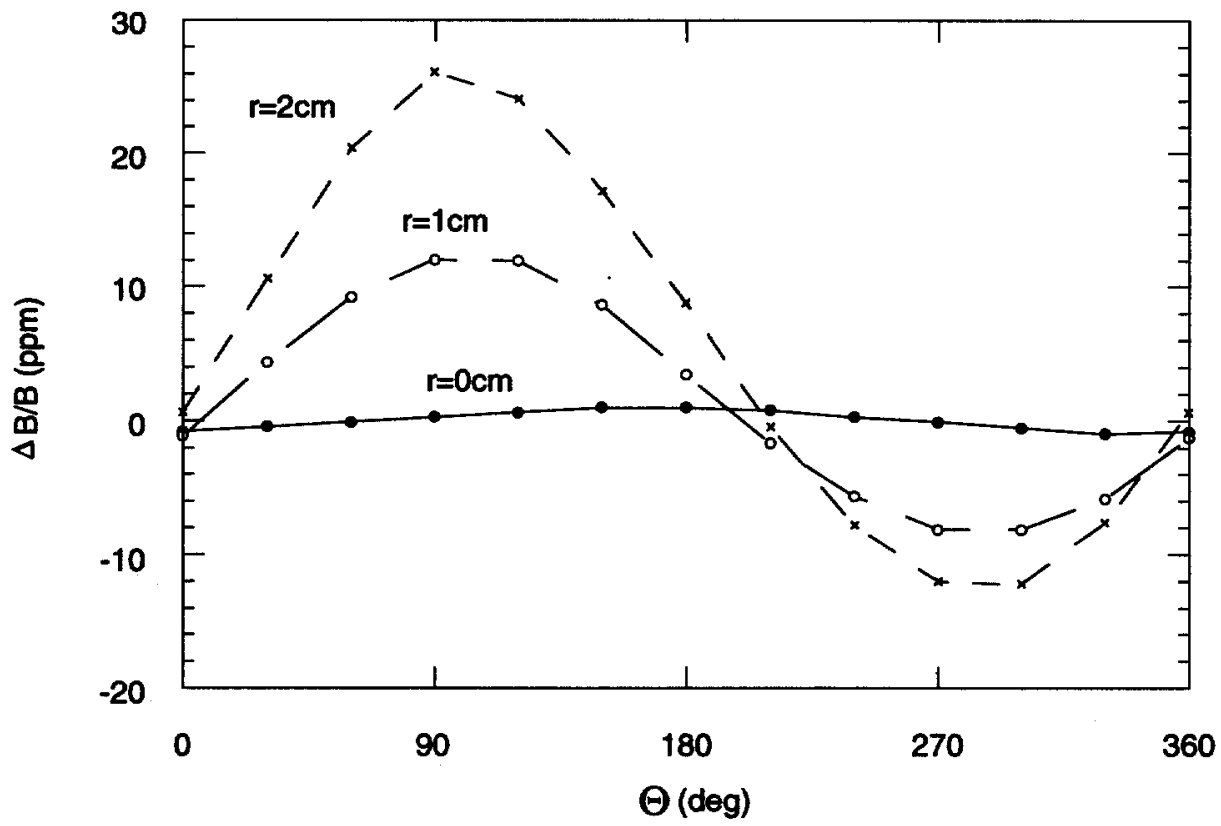

Figure 2.11 Magnetic field variation with azimuthal angle. 0 and $180^{\circ}$ define the vertical plane. 


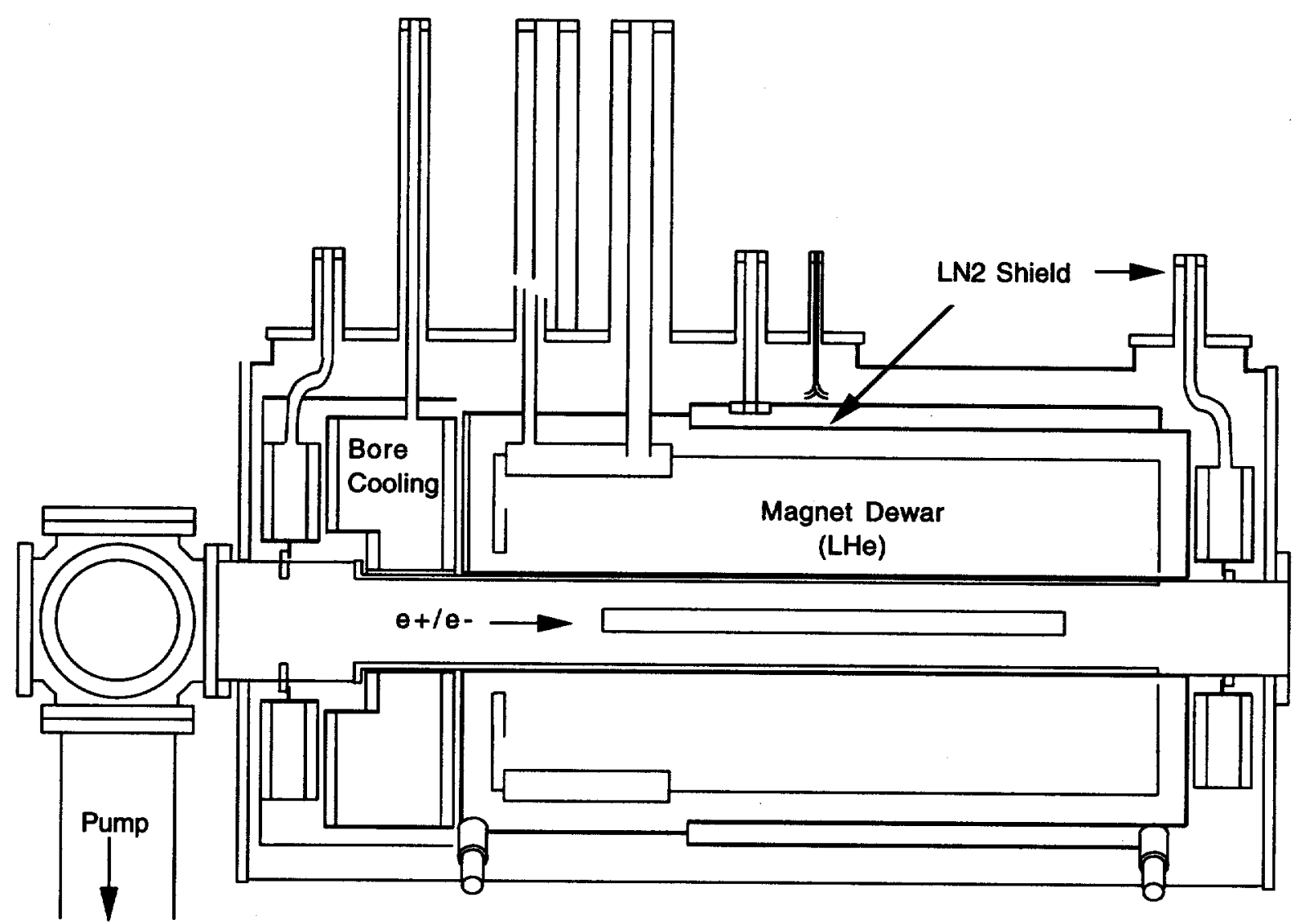

Figure 2.12 Drawing of magnet apparatus showing cryogen dewars, vacuum chamber, and approximate placement of trap electrodes.

The bore is thermally isolated from the magnet coils, allowing the temperature of the trap to be varied. Figure 2.12 shows the system of cryogen reservoirs used to cool the magnet and the bore. The bore can be cooled to $77 \mathrm{~K}$ using liquid nitrogen $\left(\mathrm{LN}_{2}\right)$ or $4.2 \mathrm{~K}$ using liquid helium (LHe) in the bore reservoir. The central section of the bore is constructed of OFHC copper for maximum temperature uniformity. Thin stainless steel tubing at either end of the bore provides thermal insulation from the end flanges to minimize consumption of cryogens. 
Dewars surrounding the stainless steel sections can be filled with $\mathrm{LN}_{2}$. This is useful for added cooling when operating at $77 \mathrm{~K}$, and is absolutely necessary to operate the bore at $4.2 \mathrm{~K}$. Additional thermal baffling would also be required in the bore at the lower temperature to minimize radiative heating and reduce LHe consumption. The present experiments are run at ambient or $\mathrm{LN}_{2}$ temperature.

The magnet radial position can be adjusted using eight struts. Four struts support the magnet at each end, and are attached to 1"-16 adjustment bolts outside of the magnet dewar. Bolt position is reproducible to within $1 / 12$ of a turn, corresponding to $100 \mu \mathrm{m}$ change in magnet position. This allows precision coaxial alignment of the magnetic field with the trap structure without the use of shim coils.

\subsection{Diagnostics}

Four basic diagnostics of the trapped particles are available: a charge collection and imaging system, gamma ray detectors, sectored 
electrodes, and a longitudinal temperature measurement.

\subsubsection{Imaging System}

The imaging system consists of a microchannel plate amplifier, phosphor screen, fiber optics, a CCD video camera, and a computer. A schematic of the assembly is shown in Fig. 2.13. Trapped particles are imaged destructively by lowering the potential on the downstream end of the trap. The particles stream out of the trap along magnetic field lines and strike the MCP. An amplified "image" of secondary electrons is acclerated to the phosphor. The light from the phosphor passes out of the vacuum system through fiber optics, and then through a 1:5 reducing fiber-optic taper. The magnetic field strength at the MCP is approximately $1 / 27$ of that in the trap. The plasma expands by the square root of the field ratio when it is dumped, in this case a factor of 5.19, and the taper reduces the image back to approximately the size of the original plasma. The taper is coupled directly to the CCD chip of the video camera. In this lensless system, the primary source of light loss is in the use of the taper. 


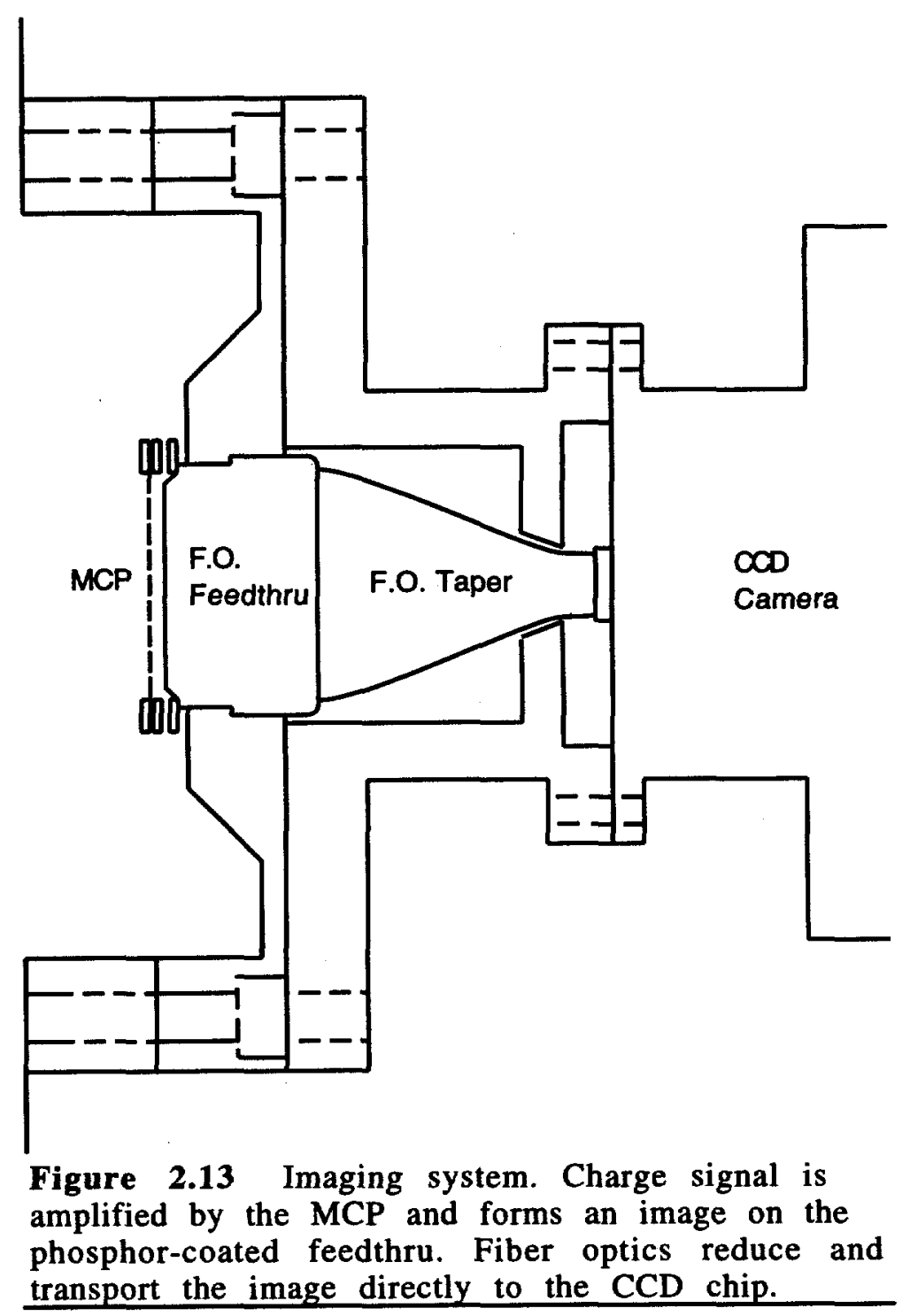

2.5.1.1 Microchannel Plate: The basis of the imaging system is a standard $40 \mathrm{~mm}$ diameter MCP. An MCP is an array of parallel miniature electron multipliers made of extruded lead glass [32]. The channels are tilted $8^{\circ}$ so particles won't pass straight through the plate. For low energy charged particle detection, the efficiency of an 
MCP is essentially equal to its open area ratio. The MCP in the trap imaging system has $10 \mu \mathrm{m}$ diameter channels with a center-to-center spacing of $12 \mu \mathrm{m}$, giving a detection efficiency of $\approx 68 \%$. The MCP assembly in the positron beamline before the trap uses two of these plates arranged in a "chevron" configuration, with channels oriented in opposite directions to maximize gain.

The gain of an MCP can be modeled by assuming the plate acts as parallel conventional discrete-stage electron multipliers [33]. The number of stages is independent of the applied voltage. This model gives a power law relationship between the gain, $G$, and the applied voltage, $V$ :

$$
G \approx \sigma_{1}\left(\frac{V}{n V_{c}}\right)^{0.5(n-1)}
$$

$V_{c}$ is the crossover potential, where primary electrons produce unity secondary emission, $n$ is the effective number of stages, and $\sigma_{1}$ is the effective gain of the first stage.

In practice, the gain is generally limited by ion feedback in the 
channels to approximately $10^{4}$ with $1000 \mathrm{~V}$ across the plate. The gain can also saturate if excessive charge is incident on the plate. In order to ensure a linear response in pulse detection, less than $10 \%$ of the charge stored in plate capacitance can be depleted in a single shot. At maximum gain, this corresponds to only a few electrons incident per channel.

The MCP is calibrated by using it first as a simple collection plate, then as an amplifier. The ratio of the two signals as a function of applied voltage is plotted in Fig 2.14 for three different incident intensities. Also plotted is a fitted curve with $n=22.24, \sigma_{1}=1.32$, $V_{c}=19.35$. The effect of saturation can clearly be seen at high voltage in all cases. The curve is consistent with relative gain data using lower density plasmas up to $V=950$. The gain change from 950 to $1000 \mathrm{~V}$ is approximately $10 \%$ lower than suggested by the curve even at the lowest incident intensity, suggesting the onset of ion feedback. 


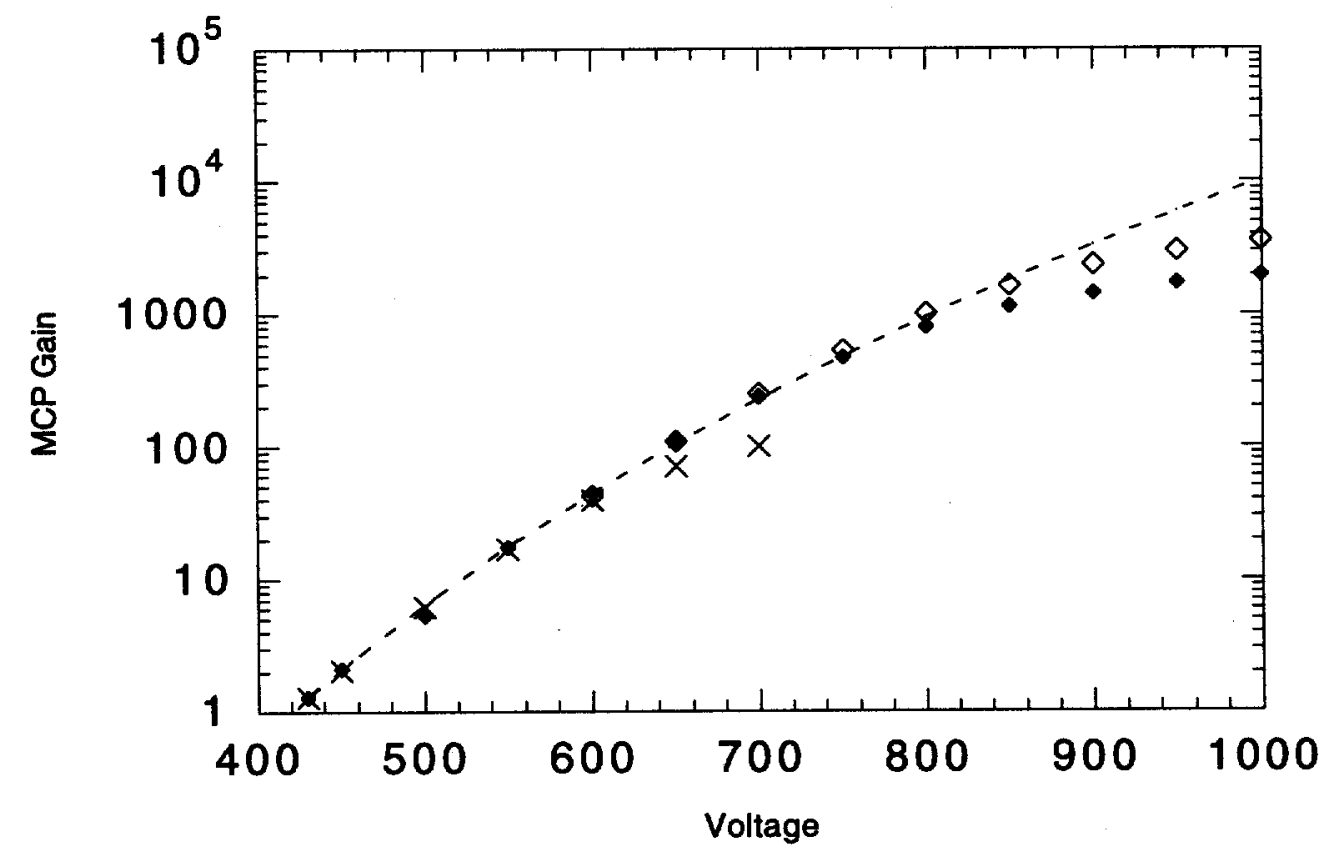

Figure 2.14 Microchannel plate gain curve. Data points show measured gain for $N_{e}=1.3 \times 10^{5}(0) 2.9 \times 10^{5}(*)$ and $5.3 \times 10^{6}(\times)$. Curve is $G=1.41 \times 10^{-28 * V 10.62}$.

2.5.1.2 Phosphor Screen and Fiber-Optics: Charge is collected on a $40 \mathrm{~mm}$ diameter fiber-optic screen coated with phosphor, aluminized to $1000 \AA$ thickness in order to shield out stray light and provide a conducting surface for charge collection. The charge signal is detected with a capacitor coupled into the bias power supply and a charge-sensitive preamplifier, as shown in Fig. 2.15. The circuit integrates the charge signal. Because the trap electrodes are switched on a timescale comparable to the particle flight time, $\sim 1 \mu \mathrm{s}$, any signal structure reflects structure in the voltage ramp. Excitation of the phosphor provides an image for measuring the radial profile of the 


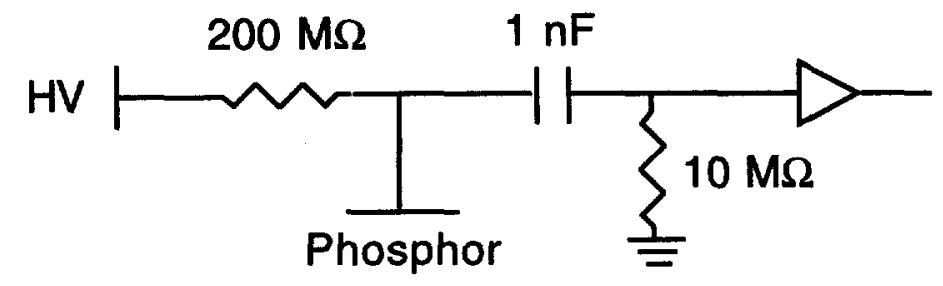

Figure 2.15 Charge detection circuit.

Preamplifier gain is $45 \mathrm{mV} / \mathrm{pC}$. System gain is reduced to $38 \mathrm{mV} / \mathrm{pC}$ by stray capacitance.

particles. The screen must be biased at least $-5 \mathrm{kV}$ relative to the back MCP voltage in order to give the electrons enough energy to penetrate the aluminum coating and create an image in the phosphor.

The fiber-optic is sealed into a 6-inch vacuum flange, and conducts the light from the phosphor out of the vacuum system. A 5:1 fiberoptic taper is coupled to the output with optical grease. Image reduction allows direct coupling of the fiber-optic to the $\mathrm{CCD}$ camera. Use of the taper results in a reduction of intensity by a factor of 25 and introduces a slight distortion of the image, most noticeably around the perimeter. Since our plasmas are generally in the central portion of the image, this distortion does not materially affect the measurements. 
2.5.1.3 Camera and Frame Grabber: The image from the phosphor is collected by a Fairchild R3000 CCD camera. The collection chip of the camera is spring-mounted with a fiber-optic cover, allowing it to be coupled directly to the taper with optical grease. The pixels of the CCD are $30 \mu \mathrm{m}$ by $18 \mu \mathrm{m}$. Due to the cancellation of the field expansion and the taper reduction, this corresponds roughly to the spatial resolution of plasma structure inside the trap. The output of the camera can be sent to a standard video monitor, or individual frames can be captured by the computer.

The CCD images are captured with a VAX 2000 workstation, where they can be stored and analyzed. Intensity information can be converted into radial density profiles, integrated along B. Image analysis software [34] permits background subtraction, averaging over several images, distance measurements, line-outs and projections, and automated location of plasma center for generating radial profiles. A typical radial profile is presented in Sec. 4.1.2.

\subsubsection{Gamma-ray Detectors}

When the trapped particles are positrons, $511 \mathrm{keV}$ annihilation 
radiation from the $\mathrm{MCP}$ is available as a diagnostic in addition to the charge signal and image. Three different detectors are utilized to measure this radiation: sodium iodide crystal $(\mathrm{NaI})$, germanium crystal $(\mathrm{Ge})$, and plastic scintillator.

The crystal detectors measure total energy deposition, providing a measurement of the total number of particles striking the MCP in a single pulse. A large $\mathrm{NaI}$ detector is placed only a few centimeters away from the MCP flange for maximum collection efficiency. The signal gain can be varied over a wide range by adjusting the bias voltage on the photomultiplier tube (PMT), as shown in Fig. 2.16, allowing measurement of low-density positron pulses and highdensity plasmas; however, the size of the $\mathrm{NaI}$ energy signal is sensitive to geometry, due to gamma rays scattering from surrounding material into the crystal with less than full energy. Consistent positioning of the $\mathrm{NaI}$ detector during calibration and use is therefore very important. The Ge crystal has better sensitivity than the $\mathrm{NaI}$, and is placed further away from the trap to minimize scattering effects and provide a better absolute particle count. Attenuation of the radiation passing through the vacuum flange and 


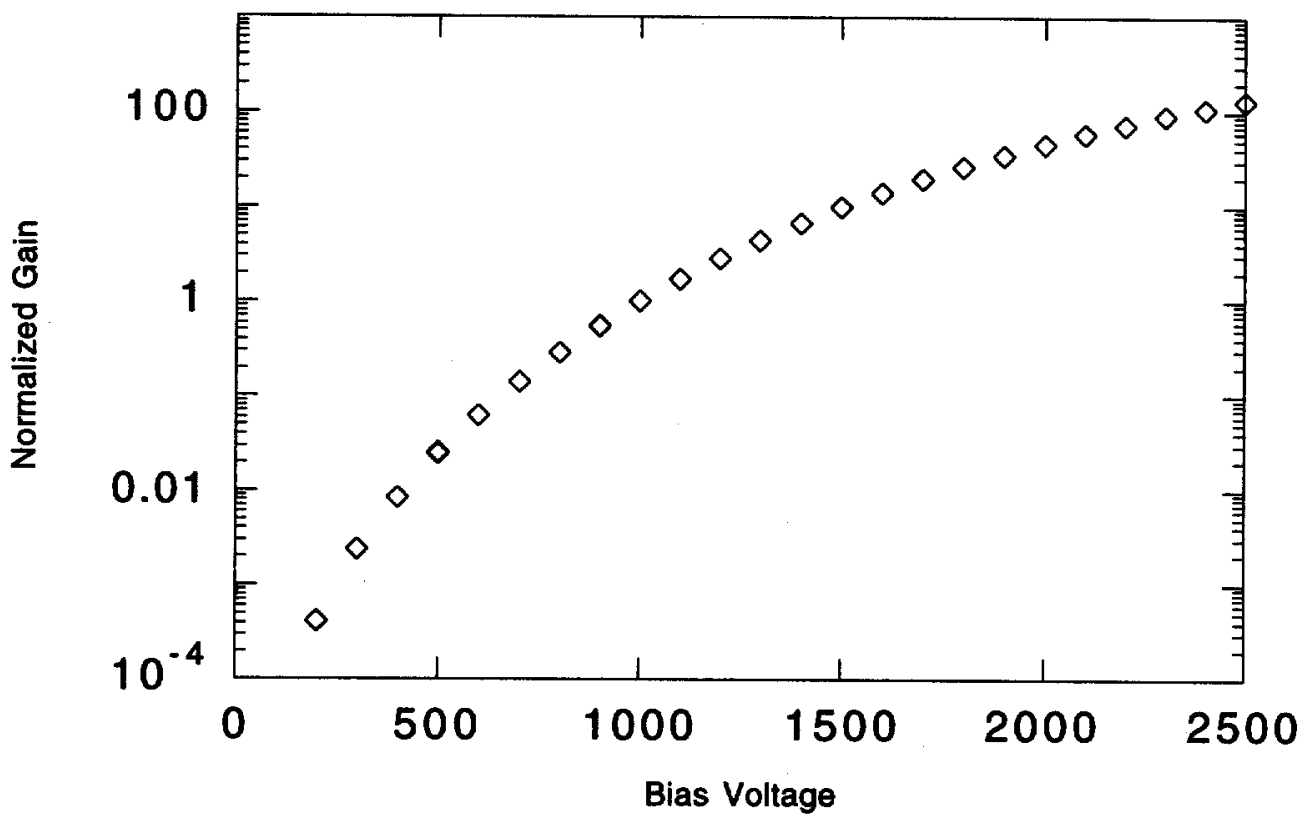

Figure 2.16 Gain curve for sodium iodide detector. Gain is normalized to $1000 \mathrm{~V}$.

camera mount to both crystal detectors is calibrated using gammaray standard sources.

Plastic scintillators are used for calibrated particle counting of the positron beam. Two cylindrical pieces, $2.5 \mathrm{~cm}$ in diameter and $2.5 \mathrm{~cm}$ long, are mounted on PMTs and heavily collimated to view a gate valve before the magnet and the MCP after the magnet. In order to prevent signal pile-up, the detectors must be placed far enough away that on average much less than one gamma ray is detected in a single beam pulse. Due to the high intensity of the beam, the upstream 
detector is placed $3-5 \mathrm{~m}$ from the gate valve. The downstream detector is placed just under $2 \mathrm{~m}$ from the MCP. The statistical nature of the particle counting limits the utility of plastic for measuring positron number during the relatively slow trapping procedure. Their principle use is in calibrating the positron beam intensity.

\subsubsection{Diocotron Motion}

As discussed in Sec. 1.3, when the charged plasma is centered in the cylindrical electrodes it remains stationary, rotating due to $\boldsymbol{E} \times \boldsymbol{B}$ drift from its own radial space charge field. When the plasma is off-axis, a second radial electric field is produced by the plasma's image charge in the conducting wall of the electrode [35]. This second field causes the plasma to orbit the electrode axis, as shown in Figure 2.17. This orbiting is called diocotron motion, and is analogous to the magnetron motion in hyperbolic-electrode Penning traps. The radial electric field for magnetron motion is provided by the trap electrodes, whereas the field in diocotron motion comes from the trapped charges.

For small displacements of an infinitely-long charge column, the 


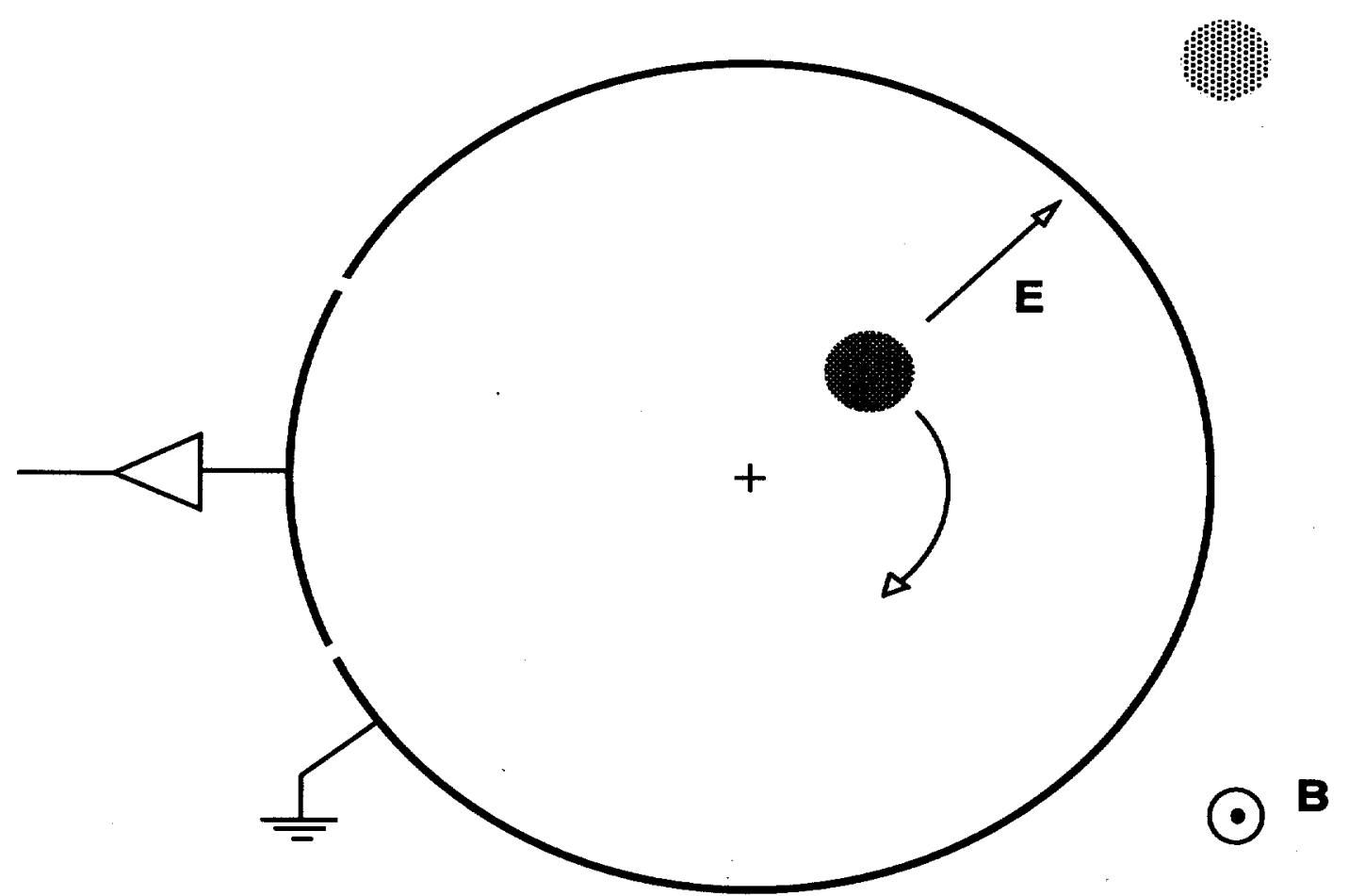

Figure 2.17 Diocotron motion. Off axis displacement of charge produces an image charge in the confining electrode. Charge orbits the axis due to E $\times B$ drift. A signal can be detected on an isolated segment of the electrode.

angular frequency of diocotron rotation is,

$$
\omega_{D}=\frac{2 c e \lambda}{R^{2} B_{z}}
$$

where $\lambda$ is the number of particles per $\mathrm{cm}, R$ is the electrode radius and $B_{z}$ is the longitudinal magnetic field strength. For typical parameters, the measured frequency is 


$$
v_{D}=4.58 \mathrm{kHz}\left[\frac{\lambda}{10^{7} \mathrm{~cm}^{-1}}\right]\left[\frac{B_{z}}{10 \mathrm{kG}}\right]^{-1}\left[\frac{R}{\mathrm{~cm}}\right]^{-2}
$$

Combined with radial information from the camera image, the diocotron frequency provides a direct measurement of the plasma density in the trap.

2.5.3.1 Limitations of Diocotron Diagnostic: The finite length of the laboratory plasma requires corrections to the infinite-column calculation, which are significant if the aspect ratio of either the plasma or the confining electrode approaches unity. A non-zero aspect ratio of the plasma produces shifts in the frequencies of all plasma modes, including the diocotron mode. A large electrode aspect ratio also changes the diocotron frequency. When the confinement electrode length approaches its diameter, field penetration from the barrier electrodes results in non-zero radial electric fields in the center of the trap. The resulting motion can be characterized as either magnetron or diocotron drift, depending on the relative strength of the electrode and plasma fields.

Figure 2.18 shows the radial electric field as a function of radius on 


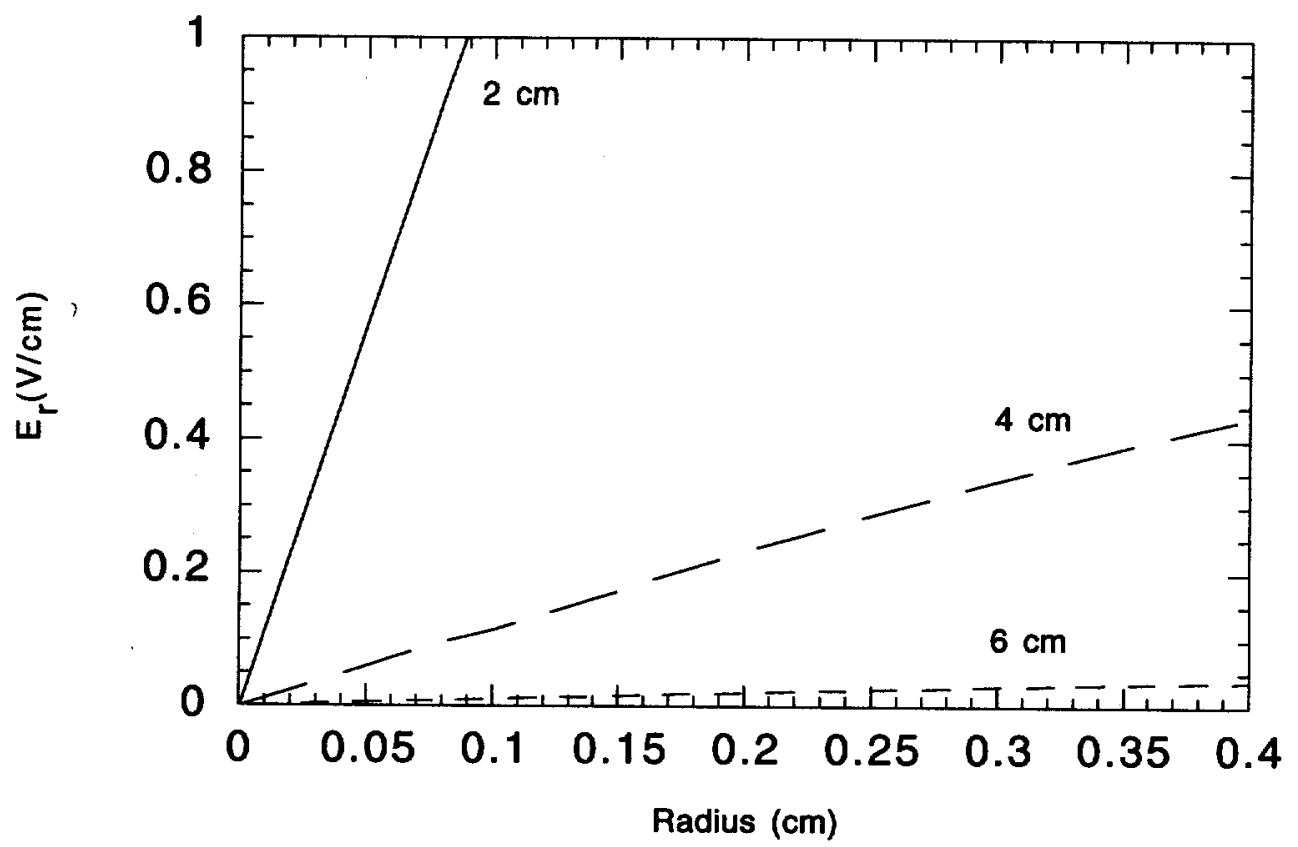

Figure 2.18 Calculated radial electric fields on trap centerline for the three shortest trap lengths. Linear field dependence mimics a uniform-density nonneutral plasma.

the centerlines of the three shortest electrodes used in this device, with $100 \mathrm{~V}$ applied to the neighboring electrodes. Note that the linear field increase mimics the ideal uniform-density plasma field. As demonstrated in Appendix C, ray-tracing calculations indicate that the bounce-averaged fields seen by a trapped particle can be substantially larger than the central field, particularly for energetic particles. As a result, the external fields encountered by particles at the ends can dominate the image charge fields even in long electrodes with no electric field in the center. In these cases, the 
diocotron frequency is ineffective as a density diagnostic.

2.5.3.2 Diocotron Measurement: Some electrodes in the trap have isolated "sectors," as shown by the broken lines in Fig. 2.17. As the image charge of the plasma passes through these sectors, it generates an oscillatory signal, which can be amplified and analyzed. The raw signal can also be fed back to another sector as a driving voltage. Depending on the relative phase of this driving signal, it can be used to either grow or damp the diocotron motion. These methods are useful with the large-radius spiral cathode. The reduction in charge using the smaller cathode reduces the signal size below noise level, rendering this measurement technique ineffective.

An alternative way to measure the diocotron frequency is with the imaging system. Varying the hold time by a few tens of microseconds causes the plasma to be dumped at a slightly different place in its orbit. The total change in hold time required to return the image to its original position is the diocotron period. As hold time increases to tens of diocotron orbits, slight variations in the timing of the control electronics result in a loss of phase between the charge dump and 
the diocotron orbit, and the image no longer appears consistently in the same location for repeated dump cycles. Therefore, the imaging technique is only useful for measuring the orbit frequency soon after injection. A single impulse on a sector can also be used to push a centered plasma off axis, as long as the pulse width is much shorter than a diocotron period, allowing use of the imaging technique at later times.

2.5.3.3 Magnetic Field Alignment: The imaging technique is particularly useful during initial alignment of the magnetic field. When the electron gun, trap electrodes, and magnetic field are all optimally aligned, there is little or no initial diocotron motion throughout the trap. During alignment, $6 \mathrm{~cm}$ electron plasmas are captured in segments at the front, middle, and rear of the trap. The magnet is moved with the 8 adjustment struts in order to minimize the diocotron orbit size. With this technique, the magnetic field can be aligned parallel to the trap electrodes to within the limit imposed by the $1 \mathrm{~km}$ radius curvature of the magnet coil itself, with diocotron orbits less than $0.1 \mathrm{~mm}$ diameter. Note that this alignment technique is independent of whether the off-axis motion is driven by diocotron 
or magnetron fields.

\subsubsection{Temperature Measurement}

The longitudinal temperature of the trapped plasma can be measured by linearly ramping down the potential on the downstream confining electrode instead of switching it quickly to ground [36]. Particles escape over the barrier when their kinetic energy satisfies:

$$
\frac{1}{2} m v_{z}^{2}\left(r, V_{d}\right)=-e\left[V_{d}-\Phi\left(r, V_{d}\right)\right]
$$

where $\Phi\left(r, V_{d}\right)$ is the space charge at the particle's radius when the dump electrode is at potential $V_{d}$. This assumes the particle is at the barrier and travelling in the correct direction at the time. In order to avoid distortion due to the bounce time of the particles, the dump potential must be ramped slowly, $d V_{d} / d t \ll k T / \tau_{b}$. In the current system, the space charge and radial dependence can be ignored due to the small radii and low particle densities studied.

Assuming the particles in the trap are in thermal equilibrium, the charge signal is the integral of a Maxwellian distribution, 


$$
Q\left(r, V_{d}\right)=\frac{2}{\sqrt{\pi}} \int_{v_{z}}^{\infty} \exp \left[-v_{z}\left(\mathrm{r}, V_{d}\right) / \sqrt{2} v_{t h}\right]^{2} d v=\operatorname{erfc}\left[v_{z}\left(r, V_{d}\right) / \sqrt{2} v_{t h}\right]
$$

where $Q$ is the total charge dumped, and $v_{t h}$ is the thermal velocity.

This function can in principle be integrated over the radial density distribution to calculate $Q\left(V_{d}\right)$. Considering only the high energy tail of the distribution, $v_{z} \gg v_{t h}$, the complimentary error function can instead be expanded for large argument to simplify the integration. Taking the logarithm of the charge and differentiating with respect to $V_{d}$ gives,

$$
\frac{d \log Q\left(V_{d}\right)}{d V_{d}}=\frac{e}{k T_{z}}\left\{1+\delta\left[n(r), v_{z} / v_{t h}\right]\right\}
$$

where $\delta$ is a first-order correction that depends only weakly on the exact density distribution. In general, $\delta=0.05$ gives a result accurate to $5 \%$.

The experimental procedure for temperature measurement is then to take the logarithm of the charge signal, in this case with the math functions of a digital oscilloscope. The ratio of the electrode ramp 


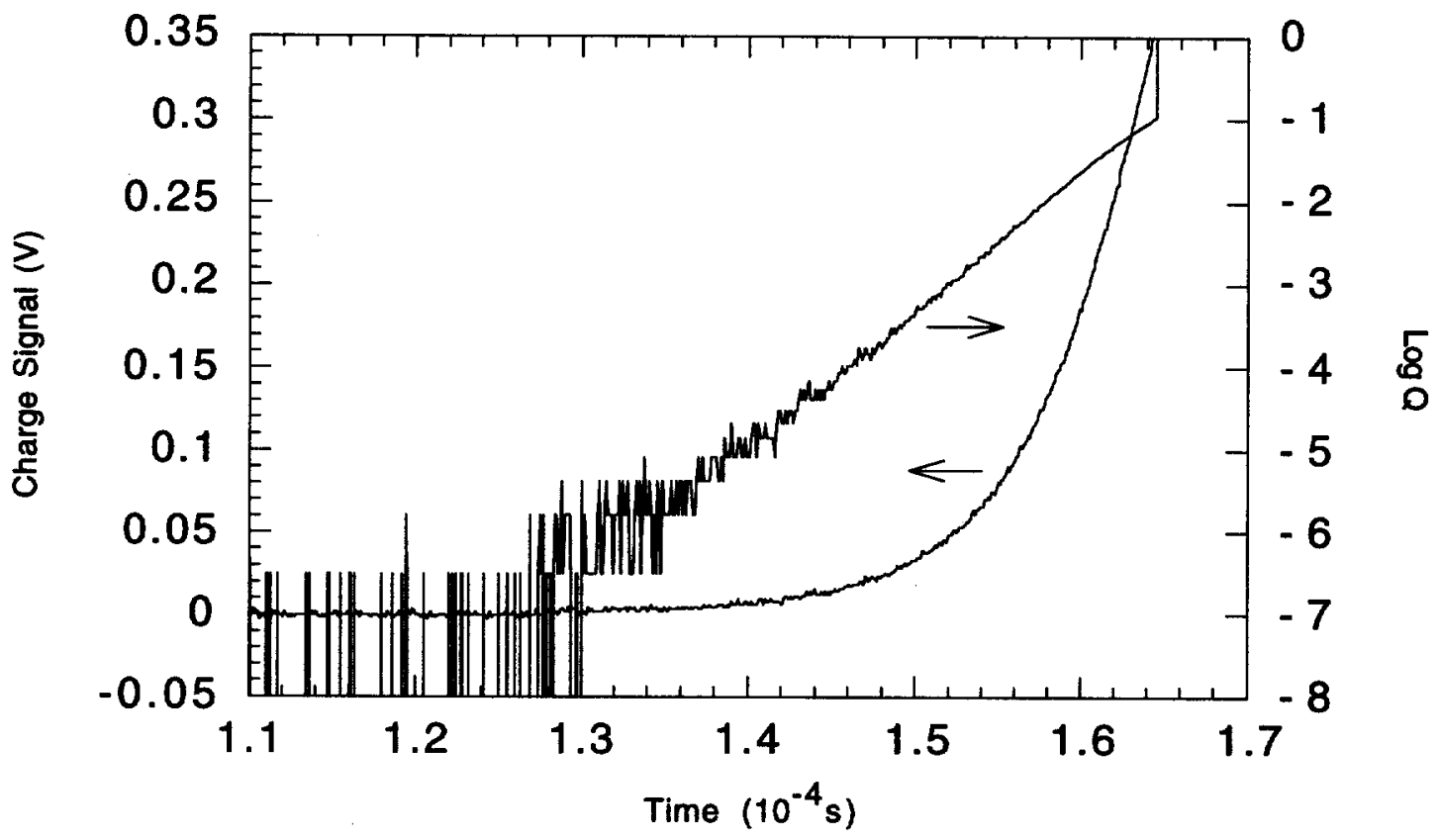

Figure 2.19 Longitudinal temperature measurement. Charge signal resulting from a slow ramp of trap barrier voltage, and logarithm of that signal.

Temperature is calculated from the ramp rate and slope of the log trace.

rate over the charge logarithm then provides a direct measure of the longitudinal temperature. Figure 2.19 shows a typical charge signal and the straight-line logarithm.

\subsection{Control Electronics}

Figure 2.20 summarizes the electronics used to control the trap. An IBM-PC compatible desktop computer is used to set the timing [37]. This timing pattern is then downloaded to a CAMAC memory module. 


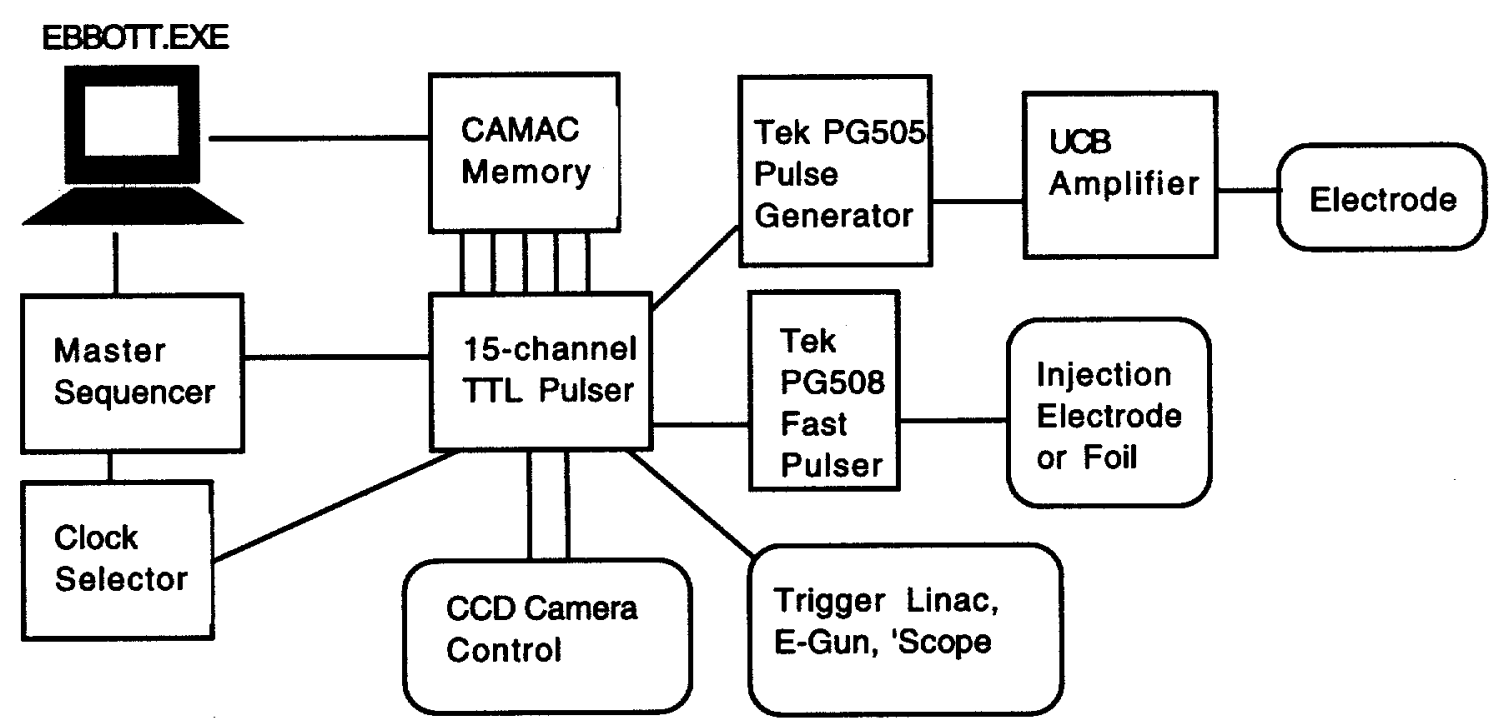

Figure 2.20 Trap control electronics. Timing pattern is loaded into CAMAC memory module. Sequencing unit steps through pattern. TTL pulses control trap operation.

A custom-made sequencing unit steps through the timing and drives

16 TTL channels that trigger the accelerator, CCD camera, frame grabber, oscilloscope, and voltage supplies for the trap electrodes and electron gun. The clock selection unit switches between clock rates $(1 \mathrm{~Hz}-100 \mathrm{kHz}$ ) within the timing pattern to allow control on multiple timescales. For long plasma lifetime measurements, sequencing is halted and a CAMAC timer triggered, allowing hold times ranging from $1 \mathrm{~ms}$ to several hours.

Control signals for the trap electrodes trigger Tektronix pulse generators. These provide variable, linear rise and fall rates for 
shaping the voltage pulses, and can be used to drive electrodes directly if low voltage pulses from $0 \mathrm{~V}$ are required. For pulse amplitudes up to $240 \mathrm{~V}$ with variable high and low levels, the pulses are sent through amplifiers designed at UCB.

\subsection{Trap Operation}

Particles can be injected into a Penning-Malmberg trap from either a continuous or pulsed source. Continuous-beam injection from the electron source is utilized in this trap to make comparisons to electron work done with other similar traps. The positron source is inherently pulsed, and the electron gun can be used in a pulsed mode in order to perform off-line tests of positron injection schemes. To avoid confusion in the following sections, diagrams of trap potentials are always shown assuming a positively-charged trap particle.

\subsubsection{Continuous Electron Source Injection}

In continuous-beam mode, the trap cycle is as shown in Fig. 2.21. An electron cloud is formed between the cathode and the downstream trap electrode. This cloud is then pinched off by raising the potential 
a)

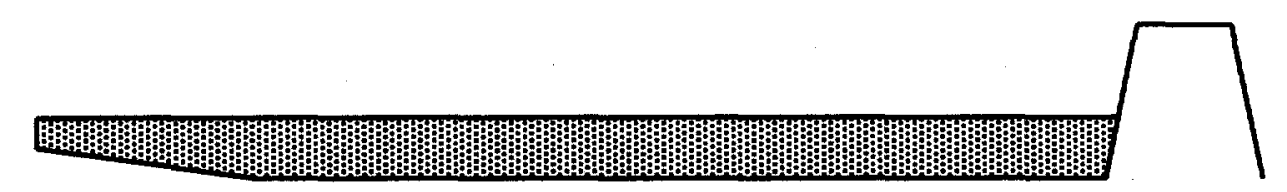

b)

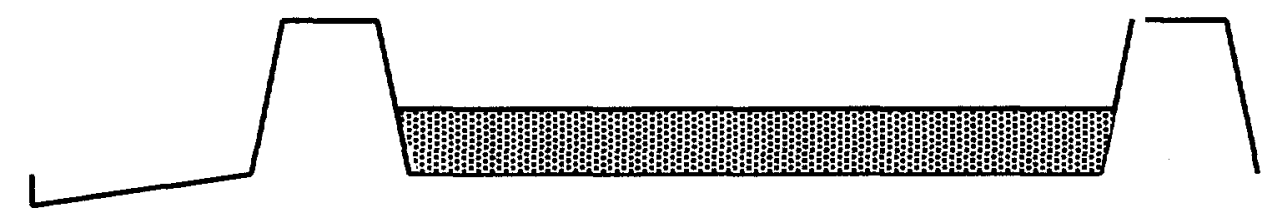

c)

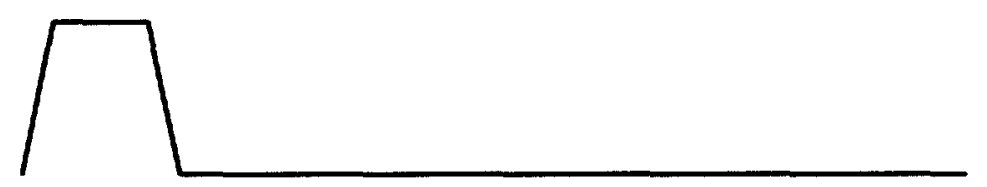

Figure 2.21 Potential structure during continuous beam electron trapping. a) Injection; b) Hold; c) Dump. The vertical line to the left represents the electron source.

of the upstream electrode. The trapped plasma typically contains $10^{5}-10^{7}$ electrons in a Gaussian radial profile with an initial radius of $0.1 \mathrm{~mm}$.

After the formation of the plasma, the cathode is biased positive. This turns off the beam and clears the electrons from the region between the gun and the trap. If this last step is not followed, the stray electrons undergo radial transport in the fringe magnetic field before the next injection phase. The characteristics of the injected plasma then vary depending on the time between injections. This can 
a)

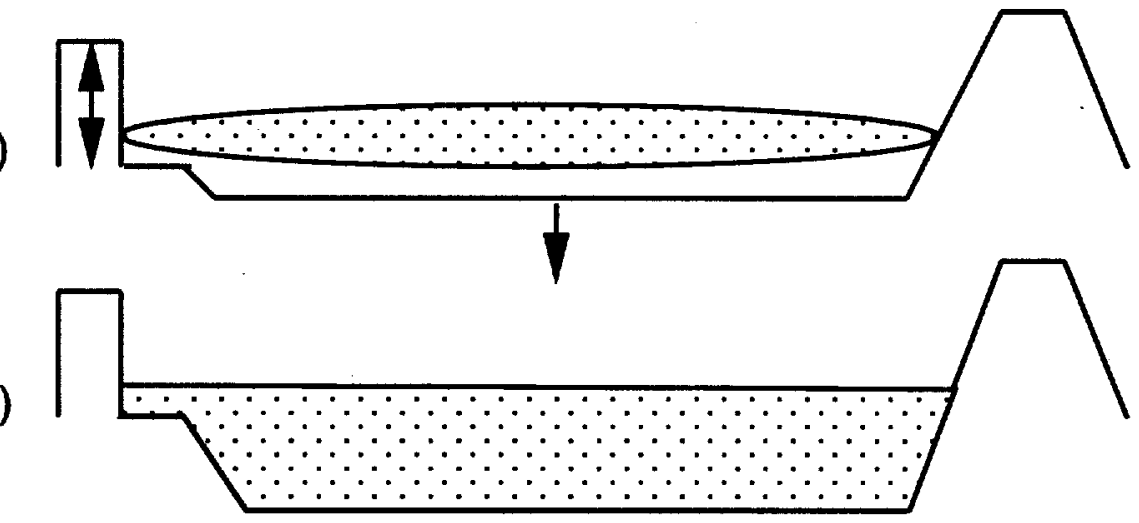

c)

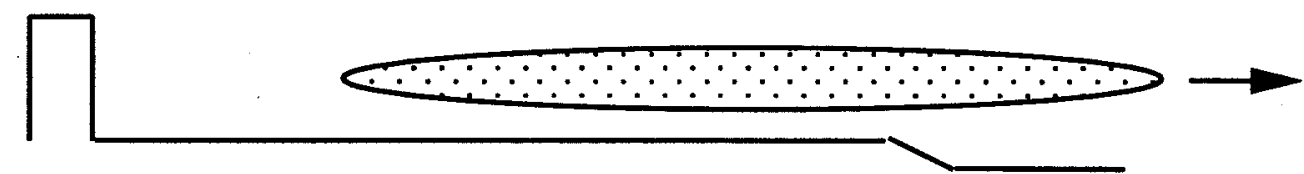

Figure 2.22 Pulsed injection, consisting of a) accumulation, b) hold, and c) dump.

give a false impression of evolution of the trapped plasma with increased hold time. In order to achieve consistent plasma characteristics, the gun bias must be negative for a fixed period of time prior to injection, independent of plasma hold time. The injected plasma density and radius is then reproducible to within a few percent.

\subsubsection{Pulsed Injection}

The basic trap cycle for accumulation of pulses is shown in Fig. 2.22. Capturing short pulses of either positrons or electrons requires faster timing than continuous beam injection. The round trip flight time of a 
$5 \mathrm{eV}$ particle in a $60 \mathrm{~cm}$ trap is only $900 \mathrm{~ns}$. For this reason, $50 \Omega$ coaxial leads are used to supply bias to the positron remoderator foil and short electrode, allowing them to be pulsed on nanosecond timescales. The remoderator foil itself is used as the injection barrier in positron injection, while the short electrode is used for pulsed electron injection.

The injection gate is triggered by the pulse signal from the accelerator electron gun or the trap cathode in electron work. A variable delay is introduced to allow for transit time difference between the signal and the particles.

During the accumulation phase, the potential of the trap region can be lowered slowly. This decreases loss by lowering the energy of particles from previous pulses relative to the potential of the injection electrode. Additional manipulations of the trapped charge can be performed between injection pulses, such as compression into shorter lengths. The variety of timing schemes used will be discussed in more detail in the next chapter. 


\section{Positron Experiments}

Initial attempts at implementation of the TOF accumulation scheme outlined in Sec. 1.2.3 have achieved only limited success, due to unexpectedly short confinement times $\tau<1 \mathrm{~s}$. The results are presented in this chapter, along with discussion of deconfinement mechanisms that are unique to positrons and to pulsed-injection TOF trapping. Confinement times $\tau>100 \mathrm{~s}$ have been achieved by modification of the injection, and storage of the particles in very short traps. While the modified techniques can contain positrons for a relatively long time, they are too inefficient to accumulate the large numbers of positrons needed for the Bhabha scattering experiment.

\subsection{Data Collection and Analysis}

The primary diagnostic for measuring the number of positrons in the trap is the NaI scintillator, discussed in Sec. 2.5.2. Rough calibration is performed by comparing the signal of a single positron beam pulse to the average pulse intensity measured using the plastic scintillators. 
It is estimated that this calibration introduces a potential systematic error in early data of up to $30 \%$ in the total positron count, resulting primarily from uncertainty in the angular position of the plastic detector. The gamma-ray attenuation varies by $30 \%$ with less than an $8^{\circ}$ change in detector position.

Accumulation data is modeled using the equation,

$$
N(t)=R \tau_{a}\left[1-\exp \left(-t / \tau_{a}\right)\right]
$$

where $R$ is the accumulation rate, and $\tau$ is the lifetime of positrons in the trap. If accumulation follows the ideal TOF formula of Sec. 1.2.3,

$$
N(t)=N_{o} \frac{t_{f}}{\Delta t}\left[1-\exp \left(-\frac{\Delta t}{t_{f}} R_{i n j} t\right)\right],
$$

then $R=N_{0} R_{i n j}$ and $\tau_{a}=\tau_{T O F}=t_{f} / R_{i n j} \Delta t$. The total accumulation should approach $N(\infty)=N_{0} t / \Delta t$, independent of the repetition rate $R_{i n j}$ Lifetime data is taken separately by halting accumulation and studying the decay of the positron signal as a function of hold time after accumulation. The resulting time spectrum is fit by a simple exponential. The intrinsic confinement time $\tau$ of the trap should 
contribute to the accumulation lifetime as $1 / \tau_{a}=1 / \tau+1 / \tau_{T O F}$.

As with most measurements on this device, data collection is a destructive process and dependent upon reproducibility. Error estimates are based on repeated measurements with identical parameters, typically $10-15 \%$ for the largest signals, increasing to over $50 \%$ for small signals resulting from partial energy deposition by scattered gamma rays. These variations appear to be consistent with the statistics of radiation detection, rather than changes in the trap performance. A full error analysis requires convolution of the Poisson distribution for photon counting with the distribution of partial-energy signals. The latter distribution is highly sensitive to placement of the NaI, and a detailed study has not been performed.

\subsection{Basic TOF Accumulation and Confinement}

The potential structure used for basic TOF trapping is shown in Fig 3.1. The linac is operated at $R_{i n j}=120$ or $480 \mathrm{~s}^{-1}$ and the trap field is $B=30 \mathrm{kG}$, giving a radiative cooling time of $0.44 \mathrm{~s}$. With the trap at 


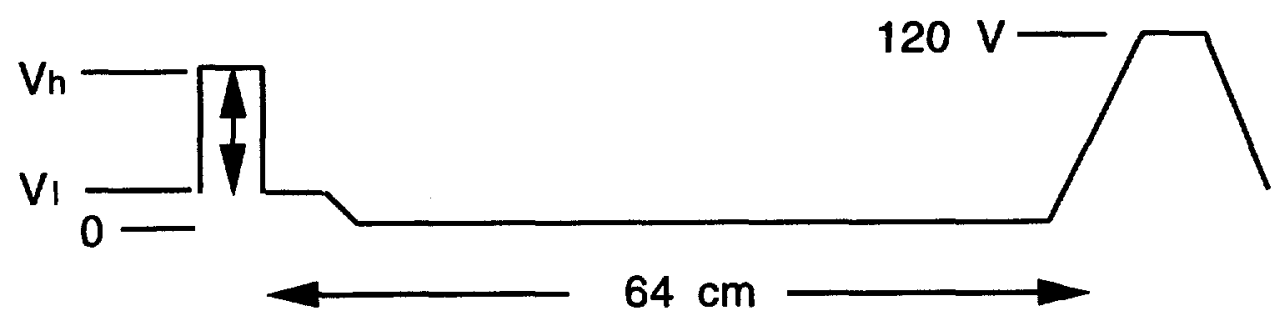

Figure 3.1 Potential structure for basic positron accumulation.

room temperature and open to the positron beam line, the ion gauge reading near the pump (See Fig. 2.12.) is $p=5 \times 10^{-9}$ Torr.

Accumulation for $V_{h}=90, V_{1}=10 \mathrm{~V}$ is shown in Fig. 3.2. The smooth curve is a least-squares fit to the model, with $R=(1.7 \pm 0.1) \times 10^{6} \mathrm{e}^{+} / \mathrm{s}$ and $\tau_{a}=49 \pm 3 \mathrm{~ms}$. Accumulation approaches $N=8.3 \times 10^{4}$ positrons for times $t \gg \tau_{a}$. The accumulation rate agrees well with the model prediction of $R=1.4 \times 10^{6} \mathrm{e}^{+} / \mathrm{s}$, but the time constant is much shorter than the prediction of $\tau_{T O F}=190 \mathrm{~ms}$. As discussed in the previous section, this suggests an intrinsic loss mechanism with a characteristic time,

$$
\tau=1 / \tau_{a}-1 / \tau_{\text {TOF }}=66 \pm 7 \mathrm{~ms}
$$

The lifetime measurement, shown in Fig. 3.3, indicates $\tau=60 \pm 5 \mathrm{~ms}$, in 


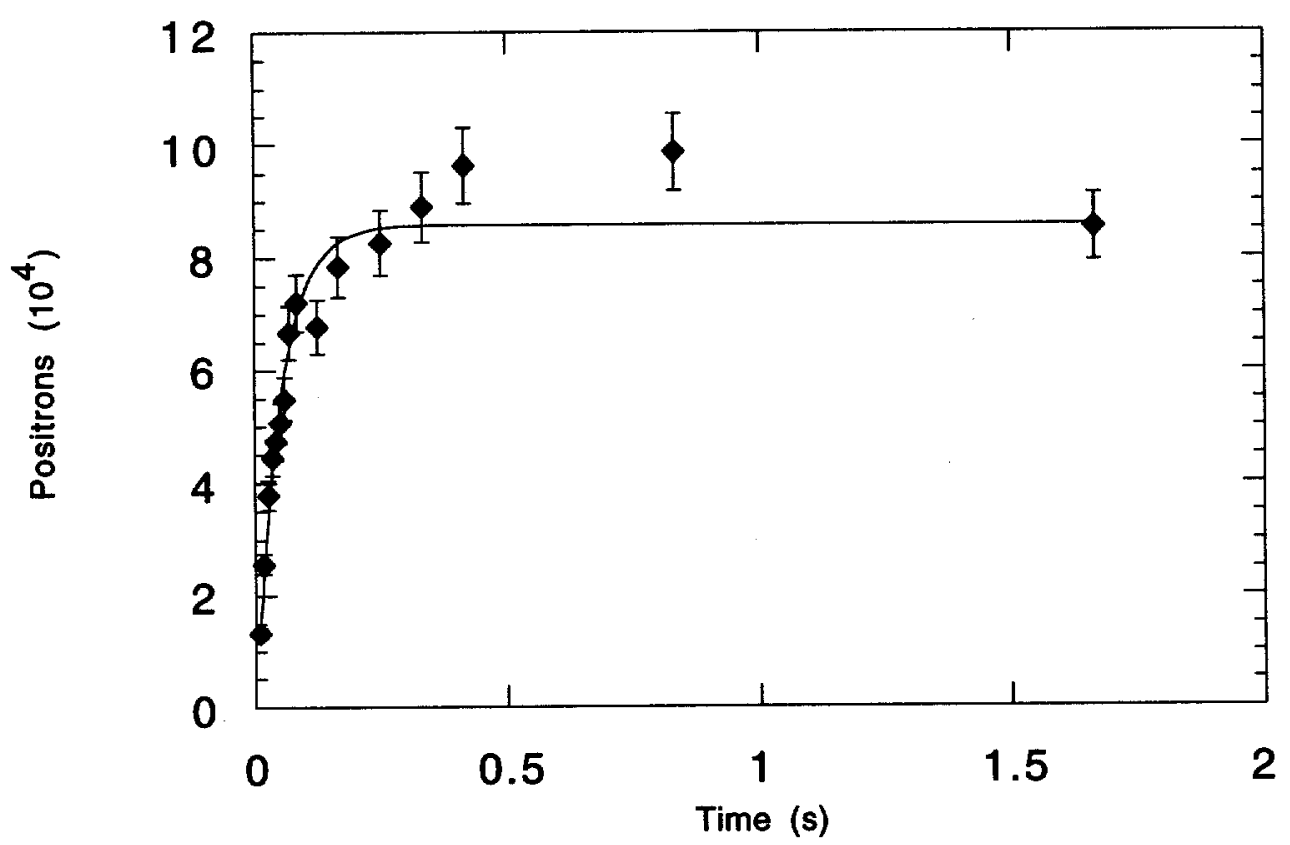

Figure 3.2 Positron accumulation. $B=3 \mathrm{~T}, \mathrm{~L}=64 \mathrm{~cm}, \mathrm{~T}=300 \mathrm{~K}, \mathrm{R}_{\mathrm{inj}}=120 \mathrm{~Hz}$. Remoderator foil pulsing $90 / 10 \mathrm{~V}$ for $30 \mathrm{~ns}$. Smooth curve is least-squares fit to TOF model, $\mathrm{R}=1.7 \times 106, \tau_{\mathrm{a}}=49 \mathrm{~ms}$.

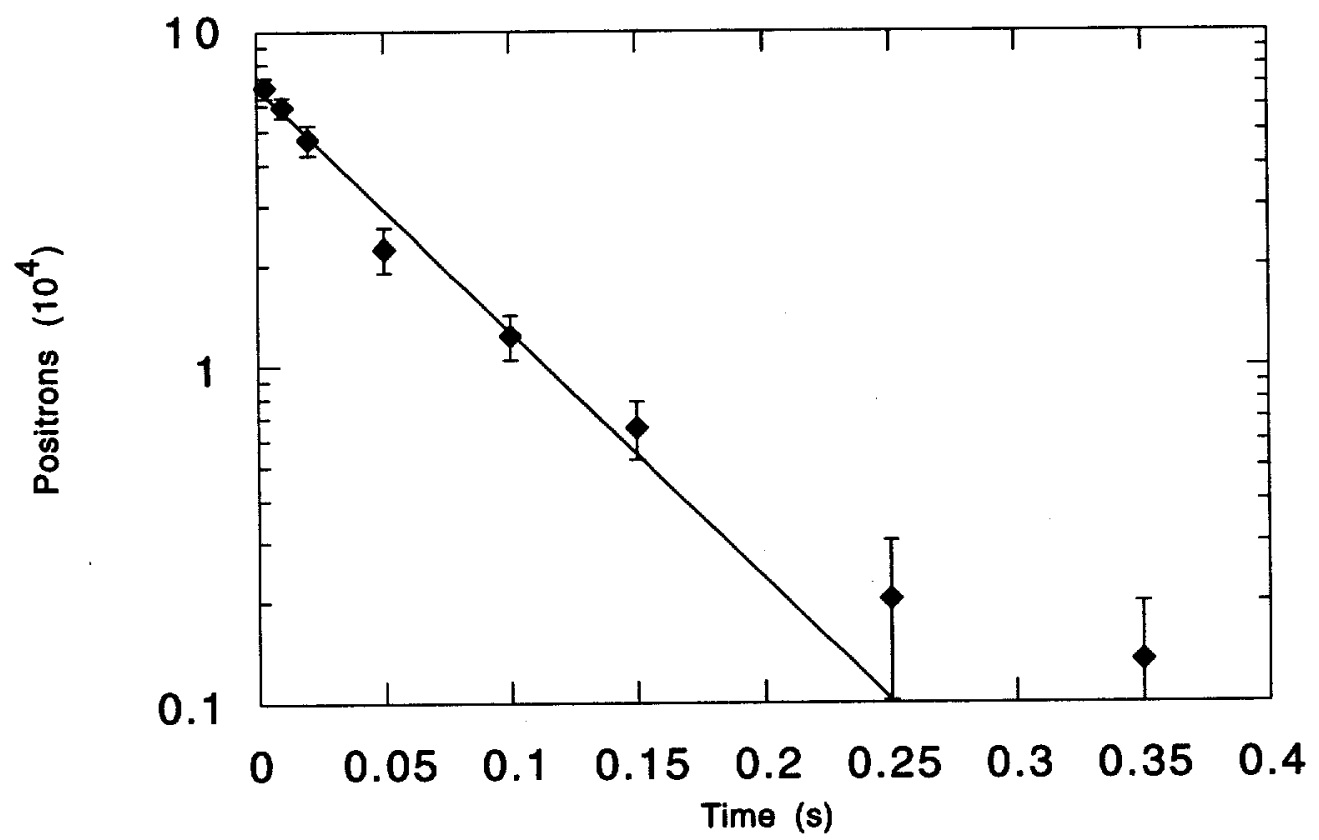

Figure 3.3 Confinement of 10 positrons pulses, accumulated with same parameters as Fig. 3.2. Solid line is a fit to an exponential, $\tau=60 \mathrm{~ms}$. 
in good agreement with the predicted value of $66 \pm 7 \mathrm{~ms}$.

The results of additional accumulation and lifetime measurements, varying $V_{h}, V_{l}, R_{i n j}$, and trap temperature $T$, are summarized in Table 3.1. Although the statistical errors in the measurements are large, several trends can be seen in the data. The value of $R$ is consistent to within $10 \%$ for fixed parameters, and increases by a factor of $\approx 3.5$ when $R_{i n j}$ is increased by a factor of 4 . This consistency lends support to the validity of the accumulation model.

\begin{tabular}{cccc|cccc}
\hline $\begin{array}{l}\mathrm{R}_{\mathrm{inj}} \\
(\mathrm{Hz})\end{array}$ & $\begin{array}{c}\mathrm{T} \\
(\mathrm{K})\end{array}$ & $\begin{array}{c}\mathrm{V}_{\mathrm{h}} \\
(\mathrm{V})\end{array}$ & $\begin{array}{c}\mathrm{V}_{\mathrm{l}} \\
(\mathrm{V})\end{array}$ & $\begin{array}{c}\mathrm{N} \\
\left(10^{4} \mathrm{e}^{+}\right)\end{array}$ & $\begin{array}{c}\mathrm{R} \\
\left(10^{6} \mathrm{e}^{+/ \mathrm{s}}\right)\end{array}$ & $\begin{array}{c}\tau_{\mathrm{a}} \\
(\mathrm{m} \mathrm{s})\end{array}$ & $\begin{array}{c}\tau \\
(\mathrm{m} \mathrm{s})\end{array}$ \\
\hline 120 & 300 & 90 & 10 & $8.3 \pm 1.0$ & $1.7 \pm 0.1$ & $49 \pm 3$ & $60 \pm 5$ \\
\cline { 2 - 7 } & & 80 & 0 & $9.1 \pm 1.2$ & $1.6 \pm 0.1$ & $55 \pm 4$ & $54 \pm 5$ \\
\cline { 2 - 7 } & 120 & 40 & $7.5 \pm 1.4$ & $1.7 \pm 0.2$ & $44 \pm 3$ & $43 \pm 4$ \\
\hline 480 & 300 & 120 & 40 & $26 \pm 2$ & $5.6 \pm 0.2$ & $46 \pm 2$ & \\
\cline { 2 - 7 } & & 20 & 10 & $37 \pm 3$ & $5.6 \pm 0.2$ & $50 \pm 3$ & $79 \pm 7$ \\
\cline { 2 - 7 } & 12 & 2 & $37 \pm 4$ & $6.1 \pm 0.3$ & $61 \pm 3$ & $73 \pm 3$ \\
\cline { 2 - 7 } & 77 & 12 & 2 & $200 \pm 40$ & $5.9 \pm 0.5$ & $337 \pm 40$ & $554 \pm 37$ \\
\hline
\end{tabular}

Table 3.1 Summary of initial positron accumulation and confinement. Values for $N, R$, and $\tau_{a}$ are from least-squares fits to the TOF accumulation model. $\tau$ is from an exponential fit to confinement data. 
One clear influence on accumulation and confinement is $V_{l}$, the depth of the potential well. This is expected to alter $\tau_{a}$ by changing the flight time $t_{f}$ With $V_{l}=0$, the close agreement between $\tau_{a}$ and $\tau$ is consistent with positron energy $<3 \mathrm{eV}$. The slow positron motion significantly reduces TOF losses, leaving the intrinsic loss mechanism as the dominant effect. Increasing $V_{l}$ to $40 \mathrm{~V}$ decreases accumulation time $\tau_{a}$ as expected, but decreases confinement time $\tau$ by the same amount, indicating an energy dependence in the confinement and continued low TOF losses.

Both effects can be attributed to positron interactions with residual gas. As discussed in Appendix B, positrons with energy $>10 \mathrm{eV}$ have a greatly increased cross section for positronium (Ps) formation. The neutral Ps immediately escapes the trap. The collision time,

$$
\tau_{c}=\left(n_{g a s} \sigma_{\mathrm{Ps}_{\mathrm{e}}} \mathrm{v}_{\mathrm{e}^{+}}\right)^{-1} \approx 1.7 \mathrm{~s}\left(\frac{p}{10^{-9} \mathrm{Torr}}\right)^{-1}\left(\frac{T}{300 \mathrm{~K}}\right)\left(\frac{\sigma_{\mathrm{Ps}}}{10^{-16} \mathrm{~cm}^{2}}\right)^{-1}\left(\frac{\mathrm{W}_{\mathrm{e}^{+}}}{10 \mathrm{eV}}\right)^{-1 / 2},
$$

is $67 \mathrm{~ms}$ for $p=5 \times 10^{-9}$ Torr and $\sigma_{P S}=5 \times 10^{-16} \mathrm{~cm}^{2}$, in excellent agreement with the measured loss rates. In addition, higher-energy 
positrons have a greater cross section for collisional cooling.

Significant scattering into the $40 \mathrm{~V}$ potential well could reduce or eliminate TOF losses. Capture by cooling is a feature of the trap design, but the design mechanism of radiative cooling would occur on a timescale 10 times longer than what is observed.

Support for the hypothesis of capture into the well is found in the effect of increasing $R_{i n j}$. Although $R$ increases roughly as expected, the corresponding decrease in $\tau_{a}$ is not seen. This is consistent with minimal TOF losses. The significance of interactions with residual gas is demonstrated by the large improvement in both accumulation and confinement at $T=77 \mathrm{~K}$. On these timescales, the primary impact of cooling the bore is a decrease in the gas pressure from $5 \times 10^{-9}$ to $1 \times 10^{-9}$ Torr. Since the pressure measurement is made far away from the cold bore, the actual change in pressure in the trap region is probably significantly greater. The resulting accumulation of $\approx 2 \times 10^{6}$ positrons in $64 \mathrm{~cm}$ is the highest total achieved to date. Additional experiments have focused on studying and improving the confinement. 
Another unexpected trend in the accumulation data is the importance of the upper-level injection voltage, $V_{h}$. Reducing $V_{h}$ results in a significant improvement in the confinement. As will be discussed in Sec. 3.4, this can be attributed to heating of the positrons by fast switching of the injection voltage. Larger $V_{h}$ results in greater heating, and the increased energy enhances positronium formation.

\subsection{Effect of Residual Gas Pressure}

The importance of positronium formation has been studied by leaking $\mathrm{H}_{2}$ and $\mathrm{He}$ gas into the trap region, and varying the positron energy in the trap. Positrons accumulate from an unremoderated $10 \mathrm{eV}$ beam in a $54 \mathrm{~cm}$ trap. The trap potential is held at +5 or $-15 \mathrm{~V}$. The $5 \mathrm{eV}$ trapped positrons are below the energy threshold for Ps formation on $\mathrm{H}_{2}$, while $25 \mathrm{eV}$ positrons are well above the threshold, and should show enhanced loss. As can be seen in Fig. 3.4, the highenergy positrons experience a rapid loss on a $10 \mathrm{~ms}$ timescale, and then decay at the same rate as the low energy positrons. The initial 


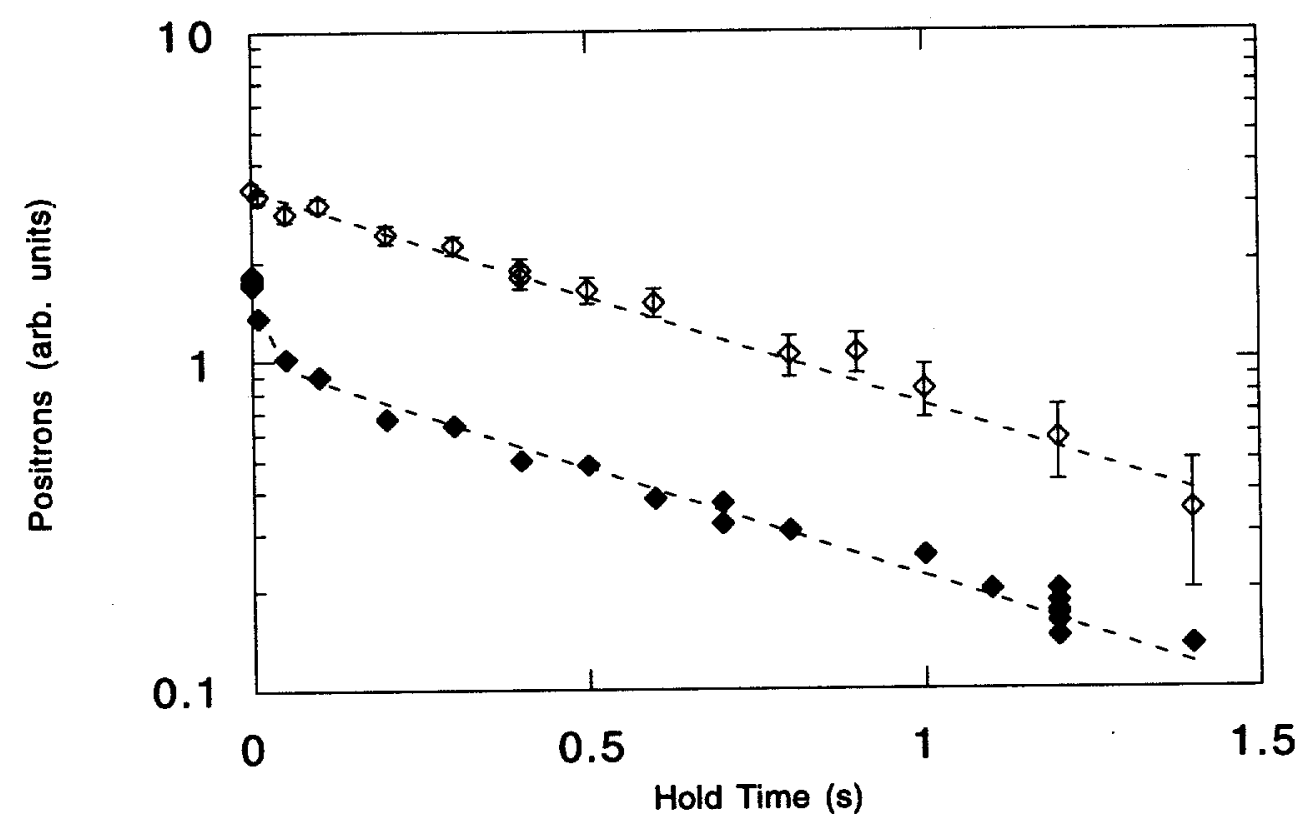

Figure 3.4 Positron lifetimes at $5 \mathrm{eV}(0)$ and $25 \mathrm{eV}(\bullet)$. Curves are fits to an exponential $(\tau=656 \mathrm{~ms})$ and double exponential $\left(\tau_{1}=13 \mathrm{~ms}, \tau_{2}=662 \mathrm{~ms}\right)$, respectively.

decay rate suggests a Ps formation cross section as high as $5 \times 10^{-15} \mathrm{~cm}^{2}$, dropping below $2 \times 10^{-17} \mathrm{~cm}^{2}$ at lower energy.

The rapid transition to the low-energy loss rate indicates that positrons are also cooling on the background gas. This behavior is consistent with the threshold model and the accumulation data. The $25 \mathrm{eV}$ positrons are also above the energy thresholds for electronic excitation of $\mathrm{H}_{2}$, resulting in the enhanced cooling. A more detailed 


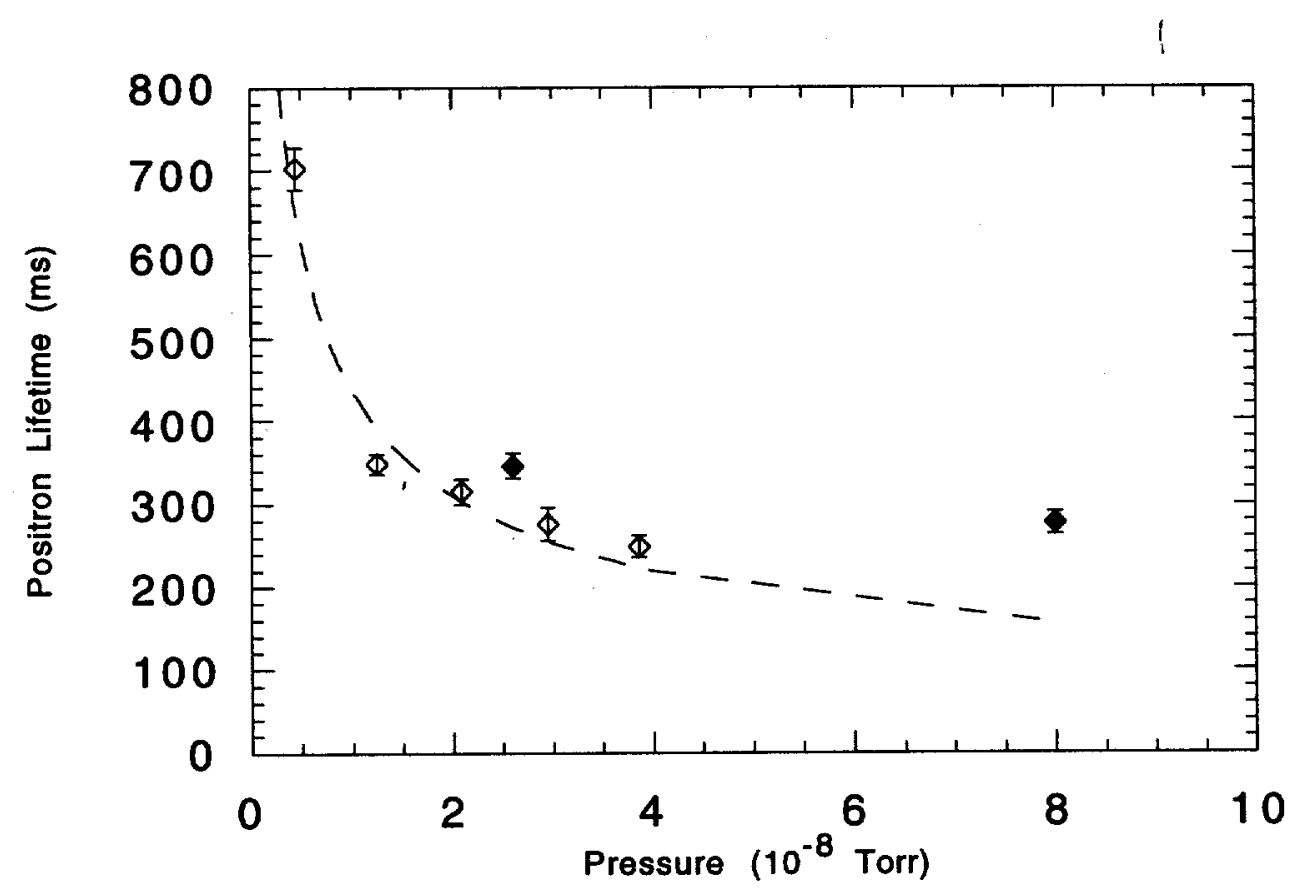

Figure 3.5 Lifetime of $5 \mathrm{eV}$ positrons as a function of $\mathrm{H}_{2}(0)$ and $\mathrm{He}(\bullet)$ pressure. Curve is a power-law fit to $\mathrm{H}_{2}$ data, $\alpha=0.50$.

discussion of positronium formation on gases is included in Appendix B.

Figure 3.5 displays positron lifetime versus total pressure of the test gases. The values shown are corrected for the ion gauge response to different gas species, assuming that the base pressure is predominantly $\mathrm{H}_{2}$. The difference in the dependence on the pressure of the noble gas $\mathrm{He}$ is suggestive, but a more extensive study would be necessary to draw hard conclusions. The plotted curve is a fit to a 
power-law, $\tau \propto \mathrm{P}^{\alpha}, \alpha=0.50 \pm 0.07$, using only the $\mathrm{H}_{2}$ data. The fact that this is not a simple inverse relationship is unusual, suggesting that particle loss is not simply dependent on the collision rate with residual gas. It is interesting to note that one electron experiment, at UCB, has exhibited a similar square-root dependence on pressure over a much broader range, though no explanation is proposed [38].

\subsection{Positron Heating}

The basic accumulation data clearly demonstrates that positron accumulation and confinement are dependent on both $V_{h}$ and $V_{l}$, which points out a significant fault in the ideal TOF model. The model assumes that the injection barrier is spatially and temporally sharp. Ideally, positrons that leave the remoderator foil have exactly the energy of the foil at the time of emission, and later reflections from the foil barrier are elastic. In practice, the foil potential extends into the trap and influences particle motion for at least one electrode diameter, $2 \mathrm{~cm}$ in this device. A $10 \mathrm{eV}$ positron travels less than 
$2 \mathrm{~cm}$ in $10 \mathrm{~ns}$. When the foil barrier is lowered for only 20-30 ns, a substantial portion of the injected positron pulse remains close enough to the barrier to get accelerated when the barrier switches to $V_{h}$. Similarly, accumulated positrons that come close to escaping when the trap is open gain energy when the trap closes. The increased energy decreases TOF efficiency by reducing $t_{f}$, and decreases confinement by heating positrons above the Ps formation threshold. Positrons can even be accelerated above the barrier potentials through repeated interactions with the foil.

The complementary interaction, in which particles are decelerated when the foil potential is lowered, also occurs. Faster particles have less interaction time with the barrier, so statistically the net effect will always be heating, even without considering the enhanced loss rates of high-energy positrons.

The significance of the heating has been demonstrated with pulsed electrons. A 30 ns pulse is applied to the bias of the electron gun to simulate the positron beam. The remoderator foil is moved slightly 


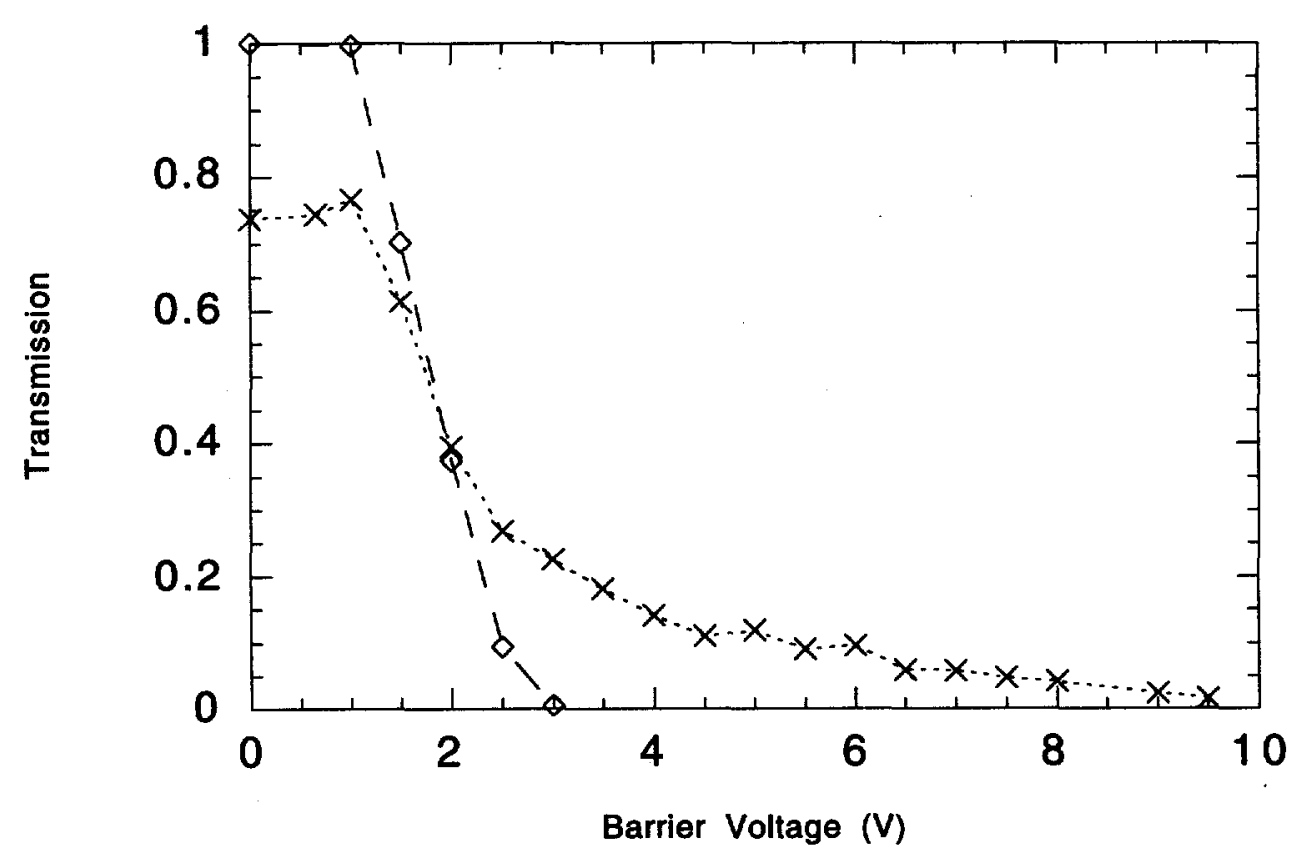

Figure 3.6 Single-pulse electron heating. Integrated longitudinal energy distribution of pulse with foil grounded $(0)$ and $V_{h}=-20 \mathrm{~V}(x)$. Lines simply connect the data points. Errors bars are about the size of the symbols.

off-axis, so the electrons pass through the supporting hightransmission mesh, but can still be captured by biasing the foil. Voltage is applied to a $6 \mathrm{~cm}$ electrode downstream to analyze the longitudinal energy distribution of the pulses. Fig. 3.6 compares the distribution of the unmodified pulse to that produced with the foil pulsing, $V_{h}=-20 \mathrm{~V}, V_{l}=0$, and $\Delta t=50 \mathrm{~ns}$. The timing delay between the gun and foil pulses is set for maximum transmission with the trap grounded, in accordance with the procedure used in positron injection. 
The unmodified electron pulse has a peak energy slightly less than $2 \mathrm{eV}$, and is completely blocked by a $3 \mathrm{~V}$ barrier. With the foil pulsing, the peak of the distribution is not significantly changed, but a substantial high-energy tail is produced. Nearly $10 \%$ of the transmitted pulse is accelerated above $6 \mathrm{eV}$ in a single pass.

Other potential sources of heating have also been investigated. The linac generates considerable rf noise, and the power supplies that maintain the trap potentials are also noise sources. To limit the effect of the accelerator, the trap electronics control the trigger pulse to the linac electron gun. After accumulation, linac injection is turned off, eliminating the large voltage pulse resulting from electron impact on the positron front end. This does not have a significant impact on confinement. In addition, electron confinement does not change when the linac is in operation, suggesting that electronic noise from the linac power system is not a significant problem.

Noise from the trap voltage supplies can be filtered with passive RC and inductive circuits, as well as active band-pass filters. None has a significant impact on positron confinement. As will be discussed in 
Sec. 4.1.4, the addition of a filter on the dump electrode in electron experiments is found to reduce the final electron temperature by $\approx 100 \mathrm{~K}$. Such low-power heating should have little effect on positron confinement, but it would be a significant concern in the lowtemperature Bhabha scattering experiment. Noise from the linac could also become an issue at that level, and could be a substantial problem for any low-energy positron work.

Manipulation of the trapped positrons can also cause heating, particularly in the case where the long positron cloud is compressed into a shorter trap section. The compression increases the temperature by the factorT $/ T_{i} \propto(L / L)_{f}^{2}$ [39]. This effect is analogous to injection heating, but the trapped particles sample the increasing voltage in the compression region many times. The enhanced positronium formation and the timing delays that must be included to allow heated positrons to cool after compression are major limitations in these experiments. 


\subsection{Low-Energy Injection into a Short Trap}

One way to improve positron confinement is to accumulate into a shorter segment. According to the empirical $L^{2}$ scaling, a $2 \mathrm{~cm}$ electrode should improve confinement by a factor of 1000 over the $64 \mathrm{~cm}$ trap, assuming interactions with residual gas are not a significant factor in the cooled trap. Accumulation in 15 such segments, with lifetimes better than $500 \mathrm{~s}$, would satisfy the design criteria for the Bhabha scattering interaction length.

Injection into a short trap is achieved by first accumulating in a longer segment, then compressing the positrons into the short segment. The potentials used in this cycle are shown in Fig. 3.7. The rather unusual structure is driven by limitations of the original trap assembly as well as heating concerns. The only useful $2 \mathrm{~cm}$ electrode is near the middle of the trap, and a defective electrode $12 \mathrm{~cm}$ into the trap limits the useful compression region. The need to minimze positron heating also limits the speed of voltage switching. 
a)

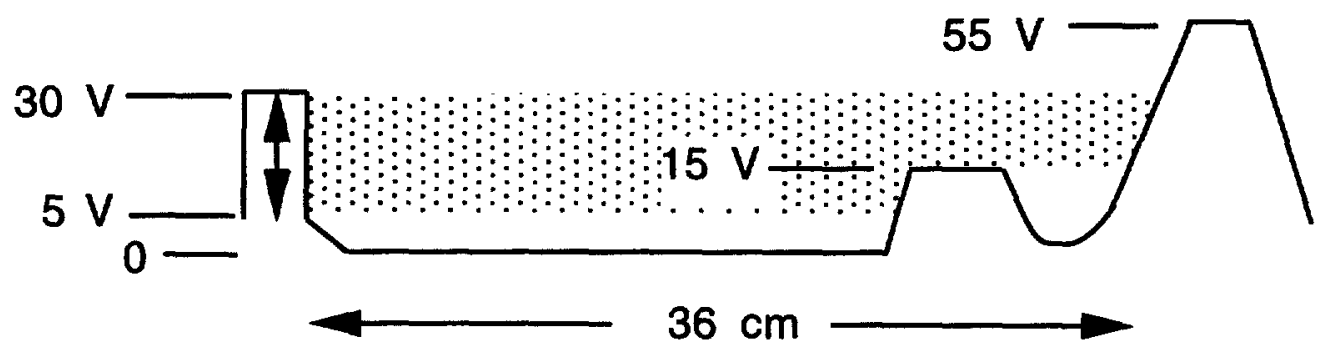

b)

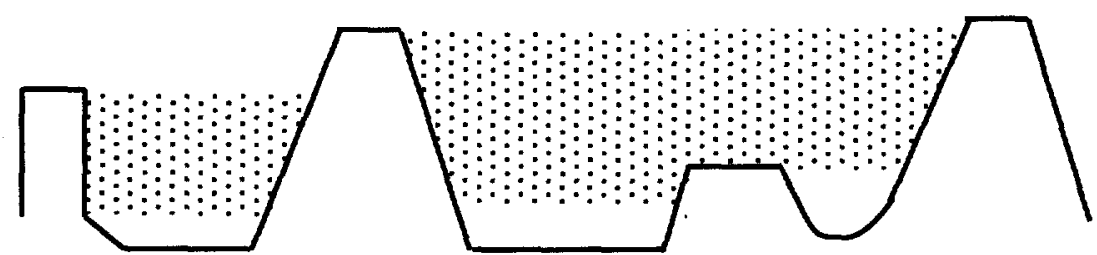

c)

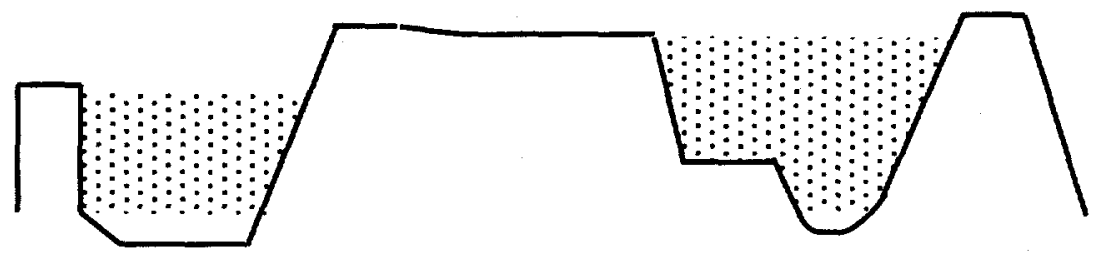

d)

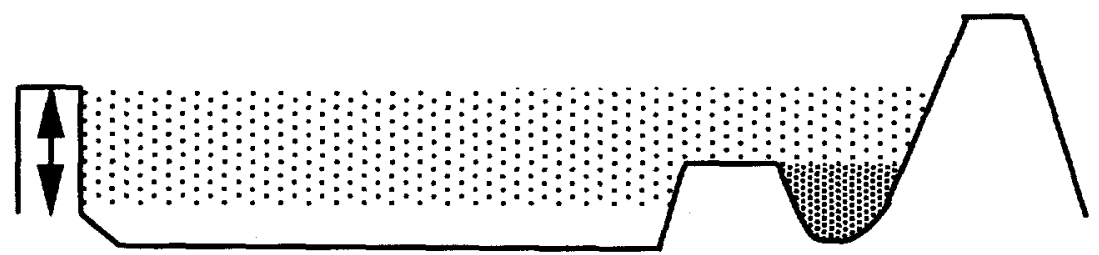

Figure 3.7 Trapping cycle for accumulation into a $2 \mathrm{~cm}$ electrode: a) accumulate 10 pulses; b) pinch; c) compress; d) accumulate.

Maintaining the $15 \mathrm{~V}$ barrier in the short trap at a constant voltage leads to more efficient accumulation that switching the barreir voltage, even though positrons must then scatter and cool in order to accumulate in the short section.

The accumulation of positrons in the $2 \mathrm{~cm}$ segment is shown in Fig. 3.8 for accelerator injection rates of 60,120 and $240 \mathrm{~Hz}$. In each 


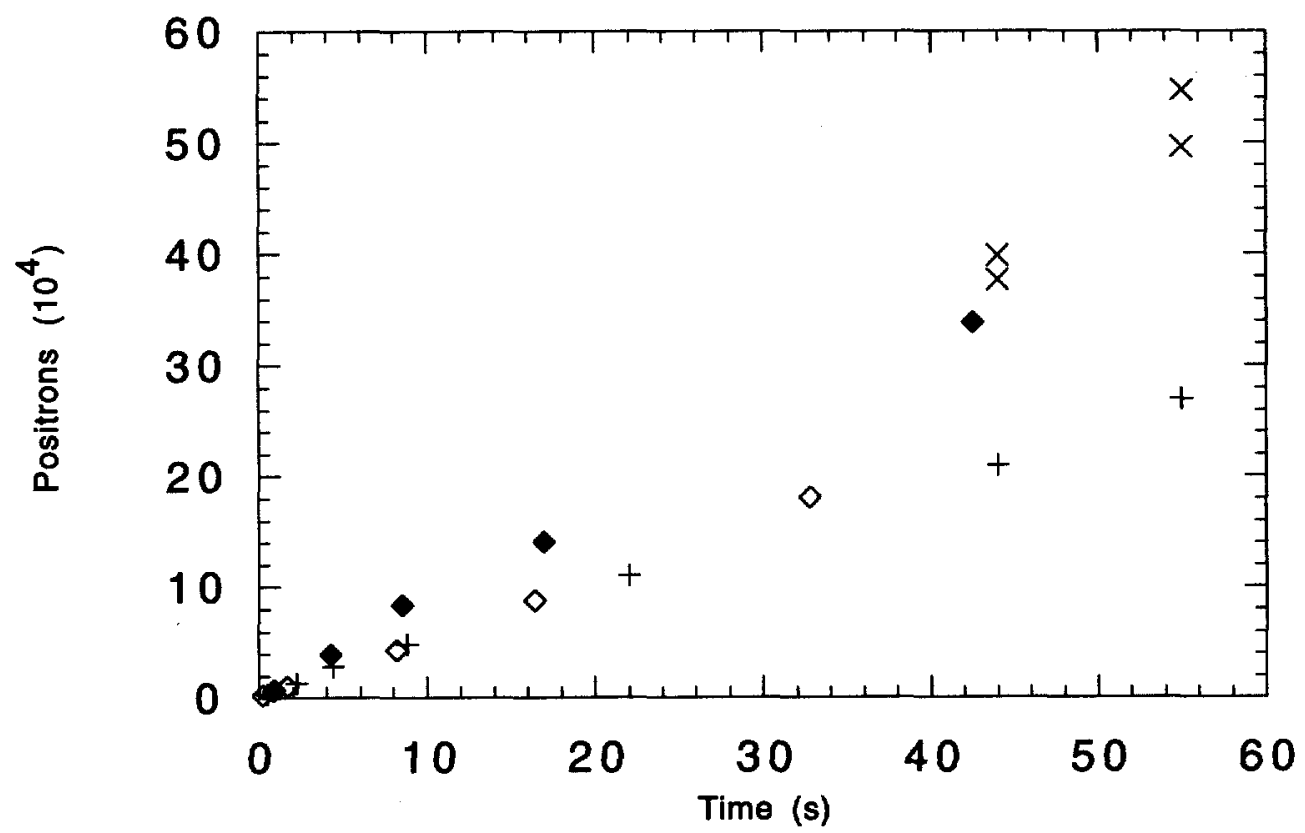

Figure 3.8 Positron accumulation in a $2 \mathrm{~cm}$ segment. ( () $60 \mathrm{~Hz}$; (৬) $120 \mathrm{~Hz}$; (+) $240 \mathrm{~Hz} ;(x) 240 \mathrm{~Hz}$, with $0.1 \mathrm{~s}$ cooling delay after compression.

case, 10 pulses are accumulated prior to compression. The $120 \mathrm{~Hz}$ accumulation rate of $8,100 \mathrm{e}^{+} / \mathrm{s}$, is not quite twice as fast as the $60 \mathrm{~Hz}$ rate of $5,400 \mathrm{e}^{+} / \mathrm{s}$. Increasing the repetition rate to $240 \mathrm{~Hz}$ decreased the efficiency back to the $60 \mathrm{~Hz}$ rate. This is likely due to the shorter accumulation time of only $42 \mathrm{~ms}$, much shorter than the cooling rate of $440 \mathrm{~ms}$. Introducing a delay of $100 \mathrm{~ms}$ during the compression phase to allow the positrons to cool into the $2 \mathrm{~cm}$ well improves the accumulation to over $9,000 \mathrm{e}^{+} / \mathrm{s}$. While the

accumulation is inefficient, it is linear in time, indicating $R_{i n j}{ }^{\gg} \tau_{a}^{-1}$. 


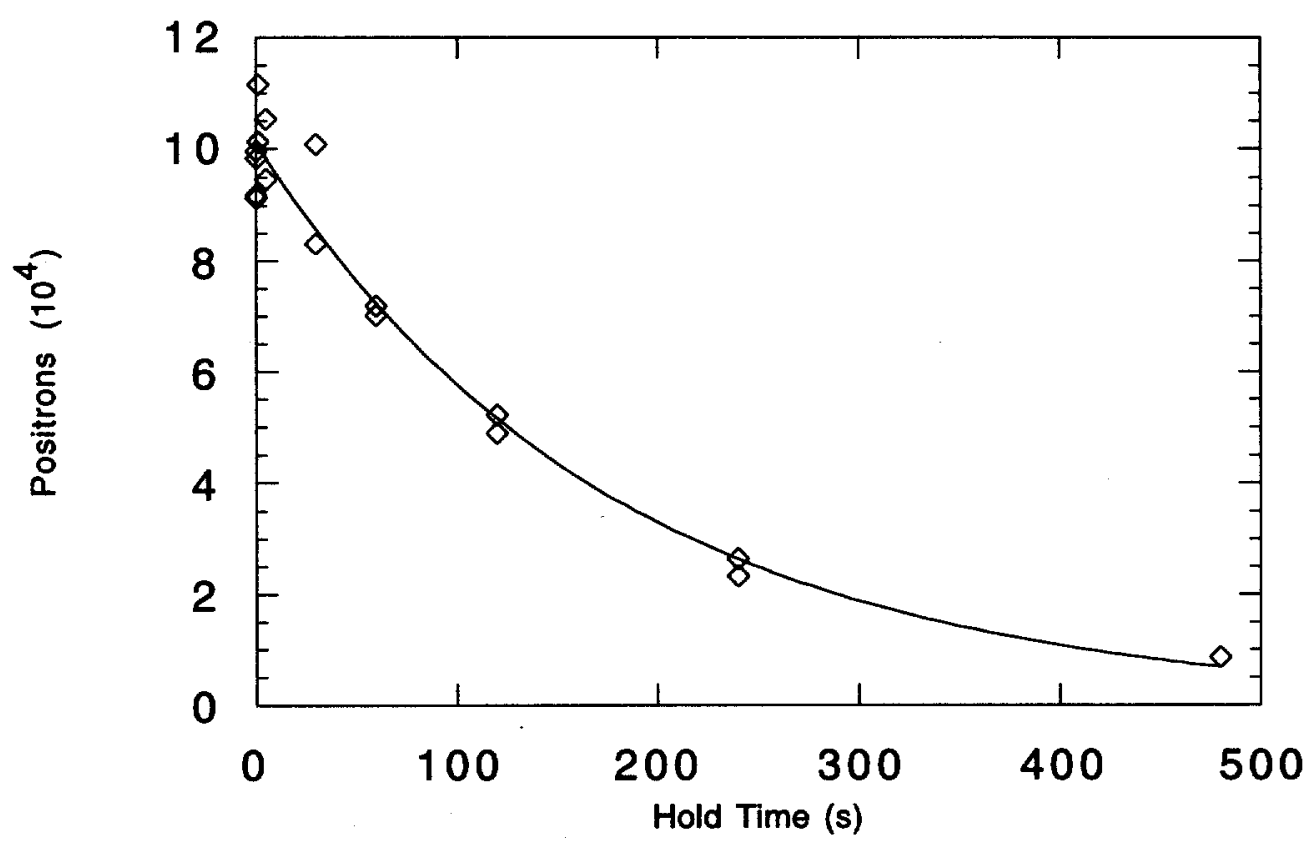

Figure 3.9 Confinement of positrons in a $2 \mathrm{~cm}$ electrode. Solid line is a fit to an exponential, $\tau=179 \pm 16 \mathrm{~s}$.

The slow loss rate is confirmed by lifetime measurements, shown in Fig. 3.9. The lifetime $\tau=179 \pm 16 \mathrm{~s}$ is still below the target of $300 \mathrm{~s}$, but would be sufficient for eventual Bhabha scattering experiments. The dramatic improvement demonstrates that the losses in longer electrodes at low temperature are due to trap effects rather than Ps formation. Imaging of the small number of positrons with the MCP is difficult, but the highest accumulation shows a central gaussian distribution with $r_{o} \approx 0.8 \mathrm{~mm}$, and a long tail extending out to $3 \mathrm{~mm}$.

The highest density achieved is estimated to be $n \approx 2 \times 10^{7} \mathrm{~cm}^{-3}$. 
a)

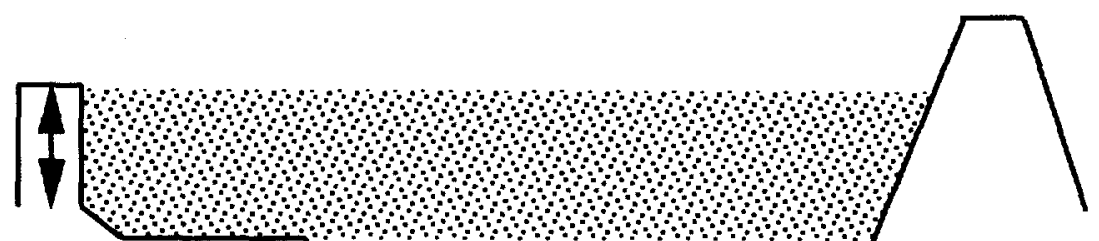

b)

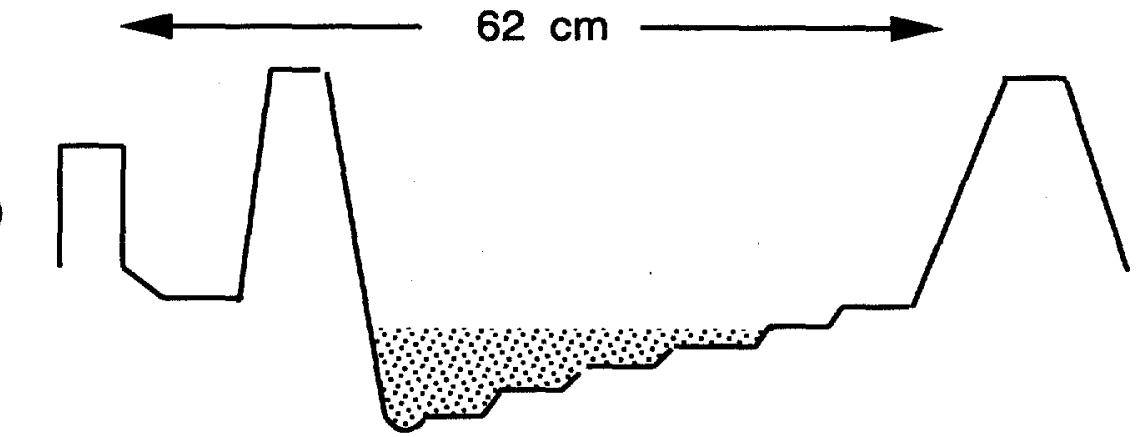

c)

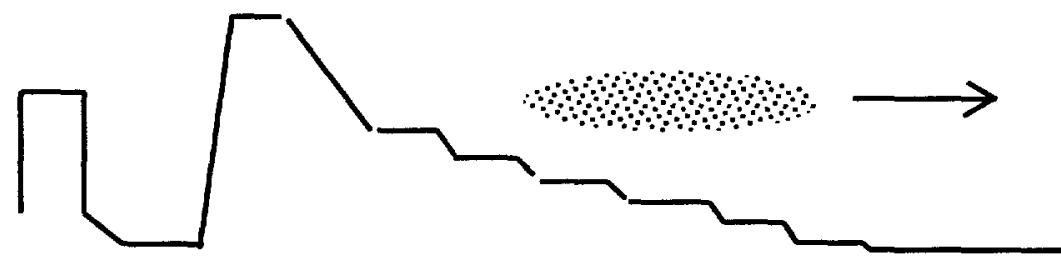

Figure 3.10 Trapping cycle for passive accumulation into a $2 \mathrm{~cm}$ electrode: a) accumulate; b) hold; c) dump. Slope is created with a resistor chain.

\subsection{New Trapping Schemes}

A number of attempts have been made to cool positrons into short segments without the heating associated with compression. A resistor chain can be used to generate a sloped potential, as shown in Fig. 3.10. The well is ramped down over several seconds during the accumulation phase. Ideally, positrons are captured in the long region, then gradually cool into the deepest portion of the well. This 
scheme has not achieved significant accumulation. Without a neutral gas to facilitate cooling, the timescale for scattering into the well appears to be too long; however, the performance is sensitive to the details of the trap voltage, ramp rate, and shape of the slope. It is possible that a useful solution for accumulating a high density of particles could be developed from this technique. For the Bhabha experiment, one short electrode does not provide enough interaction length.

In order to maximize the use of short trap segments, the trap has been reconstructed using $2 \mathrm{~cm}$ segments for accumulation, alternating with $4 \mathrm{~cm}$ barrier electrodes. The goal is to accumulate a series of short, long-lived links of positrons, using a crenelated potential, as shown in Fig. 3.11. Given the poor lifetime in long segments, this is the only configuration for accumulating a large number of positrons at high density with this trap. The long length is available for TOF capture of the beam, and the use of multiple segments should reduce the heating associated with length compression. The trapping cycle is similar to Fig. 3.7, with the electrodes immediately after the remoderator foil used to slowly 


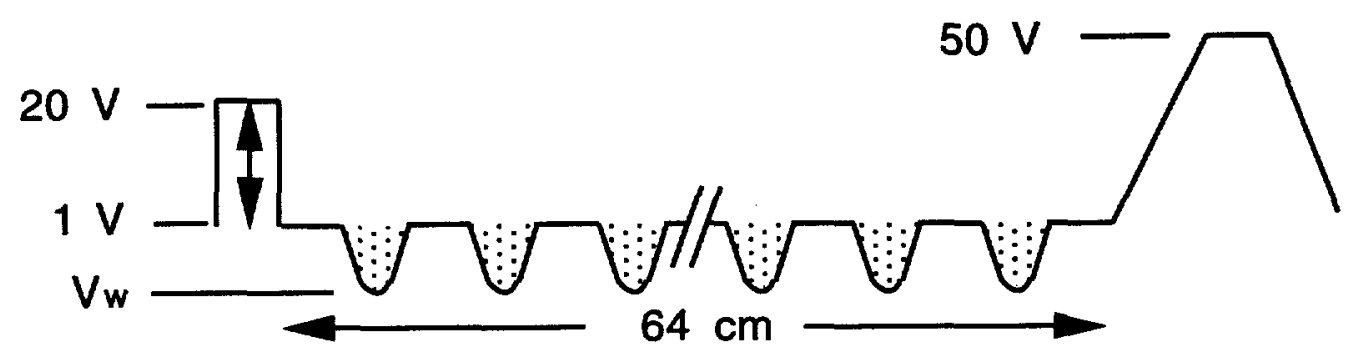

Figure 3.11 Crenelated potential structure for long-lifetime positron accumulation.

pinch off the positron cloud with a $50 \mathrm{~V}$ barrier during manipulation of the internal trap potentials.

Two schemes for accumulating into the short wells have been attempted, with limited success. First, the potential of the $2 \mathrm{~cm}$ wells is ramped down slowly through several accumulate-and-hold cycles. There is little or no accumulation into the wells in this case, though once again the full combinations of ramp rate and well depth have not been explored systematically. In the second system, the $4 \mathrm{~cm}$ electrodes are raised during the cooling period, cutting the positron cloud into short segments. The barriers are then returned to the $1 \mathrm{~V}$ level for the next accumulation phase.

Accumulation and lifetimes are difficult to measure in this system, due to problems in emptying the trap when the positrons are cold. 
When the potential of the $2 \mathrm{~cm}$ electrodes is raised to eject the positrons, the crenelated structure is inverted, with $4 \mathrm{~cm}$ wells and $2 \mathrm{~cm}$ barriers. Voltage differences of tens of millivolts between barrier segments can reflect and trap the cold positrons. Connecting the $2 \mathrm{~cm}$ wells with a resistor chain produces wells of varying depth, and the time delays in dumping the wells spread the charge signal out over several microseconds.

Qualitatively, the best accumulation and confinement occurs with $\mathrm{V}_{\mathrm{w}}=-10 \mathrm{~V}$, without spatial or temporal ramping, and the timing as follows: 1) accumulate 20 pulses, full length; 2) segment the trap by raising $4 \mathrm{~cm}$ electrodes to $40 \mathrm{~V}$ for $400 \mathrm{~ms}$; 3) drop $4 \mathrm{~cm}$ potential and hold for $100 \mathrm{~ms}$ before accumulating again. This allows the accumulation of approximately $10^{6}$ positrons in $20-30 \mathrm{~s}$, with lifetimes similar to that of the single $2 \mathrm{~cm}$ electrode. The linac is operated at $R_{\text {inj }}=120 \mathrm{~Hz}$, and no study has been made with higher injection rates. Improvement is likely to be marginal, since the timing is dominated by delays for radiative and collisional cooling of the positrons. Faster switching of the $4 \mathrm{~cm}$ potentials dramatically 
reduces accumulation in the $2 \mathrm{~cm}$ wells.

\subsection{Positron summary}

The basic physics issues associated with positron TOF accumulation and confinement are fairly well established. The problem of loss by enhanced Ps formation is unique to positrons, and was not considered in the original Bhabha experiment design. Cryogenic cooling of the trap and careful consideration of the injection timing can in principle minimize this loss mechanism. The real challenge to accumulation is the unexpectedly poor confinement properties of the trap even in the absence of Ps losses. This can be partially overcome by taking advantage of the good confinement in short traps, but the heating associated with compression can once again cause Ps losses. More important for the Bhabha scattering experiment, the time delays associated with trap manipulation lead to an unacceptable decrease in the accumulation efficiency. The cause of the poor trap performance has been explored using electrons, and will be discussed in Chapt. 4. 


\section{Electron Confinement}

The good confinement of positrons in a $2 \mathrm{~cm}$ electrode clearly demonstrates that positron confinement is not limited by interactions with residual gas. The poor confinement in longer electrodes is a consequence of more general trap properties. A systematic study of the scaling laws of this trap has been performed for comparison to similar traps, which are discussed in Chapter 1 and Appendix A. The confinement scalings with length, magnetic field, and density are found to differ substantially from the predicted behavior. In addition, there is a clear lifetime dependence on the radius of the plasma. Previous devices on which systematic confinement studies were done had only a factor of two difference between plasma and electrode radius, making it difficult to study a large range of radii.

Table 4.1 lists the parameter range of the electron plasmas studied in this device. For simplicity, "plasma" will be used to refer to the trapped electrons even though the term is not always strictly accurate. In many cases $\lambda_{D} \geq r_{p}$, particularly for small $B$, when 


\begin{tabular}{|c|c|c|c|c|c|c|}
\hline & & & Min. & Max. & Typ. & Units \\
\hline Mag. Field & B & & 500 & $6 \times 10^{4}$ & $3 \times 10^{4}$ & $\mathbf{G}$ \\
\hline $\begin{array}{l}\text { Cyclotron } \\
\text { Freq. }\end{array}$ & & $\Omega$ & $9 \times 10^{9}$ & $1 \times 10^{12}$ & $5 \times 10^{11}$ & $\operatorname{rad} s^{-1}$ \\
\hline Density & $\mathbf{n}$ & & $10^{6}$ & $10^{9}$ & $10^{8}$ & $\mathrm{~cm}^{-3}$ \\
\hline Plasma Freq. & & $\omega_{\mathrm{p}}$ & $6 \times 10^{7}$ & $2 \times 10^{9}$ & $4 \times 10^{8}$ & $\mathrm{rad} \mathrm{s}^{-1}$ \\
\hline Temperature & $\mathrm{T}$ & & $2 \times 10^{-2}$ & 5 & $8 \times 10^{-2}$ & $\mathrm{eV}$ \\
\hline $\begin{array}{l}\text { Thermal } \\
\text { velocity }\end{array}$ & & $v_{t h}$ & $6 \times 10^{6}$ & $9 \times 10^{7}$ & $10^{7}$ & $\mathrm{~cm} \mathrm{~s}^{-1}$ \\
\hline Debye Length & & $\lambda_{\mathrm{D}}$ & $3 \times 10^{-3}$ & 1.7 & $2 \times 10^{-2}$ & $\mathrm{~cm}$ \\
\hline $\begin{array}{l}\text { Cyclotron } \\
\text { Radius }\end{array}$ & & $\rho$ & $6 \times 10^{-6}$ & $1 \times 10^{-2}$ & $2 \times 10^{-5}$ & $\mathrm{~cm}$ \\
\hline Collision Freq. & & $v_{\mathrm{ee}}$ & 5 & $7 \times 10^{6}$ & $10^{4}$ & $s^{-1}$ \\
\hline $\begin{array}{l}\text { Electrode } \\
\text { Length }\end{array}$ & $\mathrm{L}_{\mathrm{o}}$ & & 2 & 52 & 6,18 & $\mathrm{~cm}$ \\
\hline Plasma Radius & $\mathbf{r}_{\mathbf{p}}$ & & $10^{-2}$ & $10^{-1}$ & $1.5 \times 10^{-2}$ & $\mathrm{~cm}$ \\
\hline
\end{tabular}

Table 4.1 Summary of trap parameters.

cyclotron cooling is negligible. In all but the shortest electrodes, $\lambda_{D}<L$, resulting in an unusual one-dimensional shielding.

\subsection{Data Collection and Analysis}

\subsubsection{Trap Operation}

Electrons are injected into the trap from a continuous beam, as 
described in Sec. 2.7.1. During injection, the cathode is biased $-10 \mathrm{~V}$. The density of the plasma is adjusted by varying the heating power to the cathode or by "stacking." Stacking involves multiple injection cycles, analogous to the accumulate-and-compress cycle described for positrons in Sec. 3.5. Stacking can also result in increased radius, limiting its use for density studies, but it is used for studies of the effect of plasma radius on the confinement in Sec. 4.6.

The trap end electrodes are biased $-35 \mathrm{~V}$. The trapping region is maintained at $-1.5 \mathrm{~V}$. The effective barrier potential is somewhat low relative to the initial electron temperature of about $5 \mathrm{eV}$, which can result in the loss of a few percent of the plasma to evaporation immediately after injection. Larger barrier potentials result in fringe fields in $2 \mathrm{~cm}$ traps that reflect the electrons, making injection impossible without also increasing the gun potential. For consistency, gun and trap potentials are the same at all lengths.

\subsubsection{Length Calculations}

As discussed in Sec. 2.5.1, the imaging system provides only the radial profile of the plasma $f(r)$ and the total charge $N$. The actual 
length must be calculated from the data, the known trap configuration, and the plasma temperature. This is done numerically by self-consistently solving Poisson's equation,

$$
\frac{1}{r} \frac{\partial}{\partial r} r \frac{\partial \varphi}{\partial r}+\frac{\partial^{2} \varphi}{\partial z^{2}}=n(r, z, T, \varphi)
$$

with the constraints,

$$
\int_{-\infty}^{\infty} \int_{0}^{\infty} n(r, z) d z r d r=N
$$

and

$$
N^{-1} \int_{-\infty}^{\infty} n(r, z) d z=f(r)
$$

Performing this calculation for every data point in over one hundred lifetime studies is impractical, so instead the length is calculated by three-dimensional interpolation over a tabulated set of solutions $L\left(T, N, r_{p}\right)$. Fig. 4.1 shows a calculation of $L(T, N)$ for a gaussian radial profile $r_{p}=0.01 \mathrm{~cm}$ in a $4 \mathrm{~cm}$ electrode [40].

The radial profile is assumed to be a gaussian, parameterized only by $n_{o}$ and $r_{p}$. Fig. 4.2 shows an actual radial profile and the gaussian fit. 


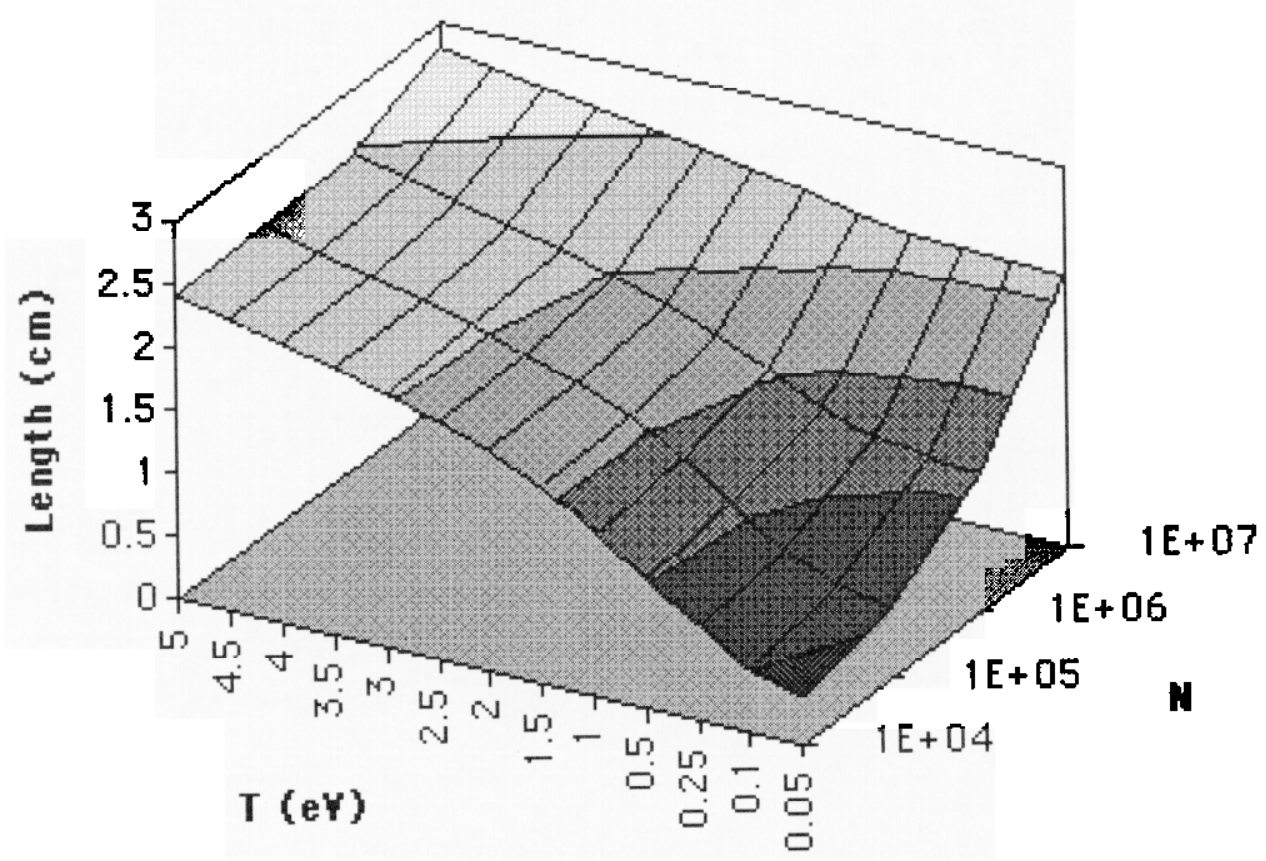

Figure 4.1 Calculation of plasma length as a function of temperature and electron number. $\mathrm{L}_{\mathrm{o}}=4 \mathrm{~cm}, \mathrm{r}_{\mathrm{p}}=0.01 \mathrm{~cm}$.

The roughly $5 \%$ disagreement in central density $n_{o}$ between the data and the fit is representative of the worst disparities seen in the data, and occurs in only a few percent of the studies. The $1 / \mathrm{e}$ radius is in general still in good agreement, and the length calculations are relatively insensitive to small variations in radial profile.

It should be noted that, although the observed radial profile does not conform to the traditional uniform-density distribution in nonneutral plasmas, it can in principle still be in thermal equilibrium. 


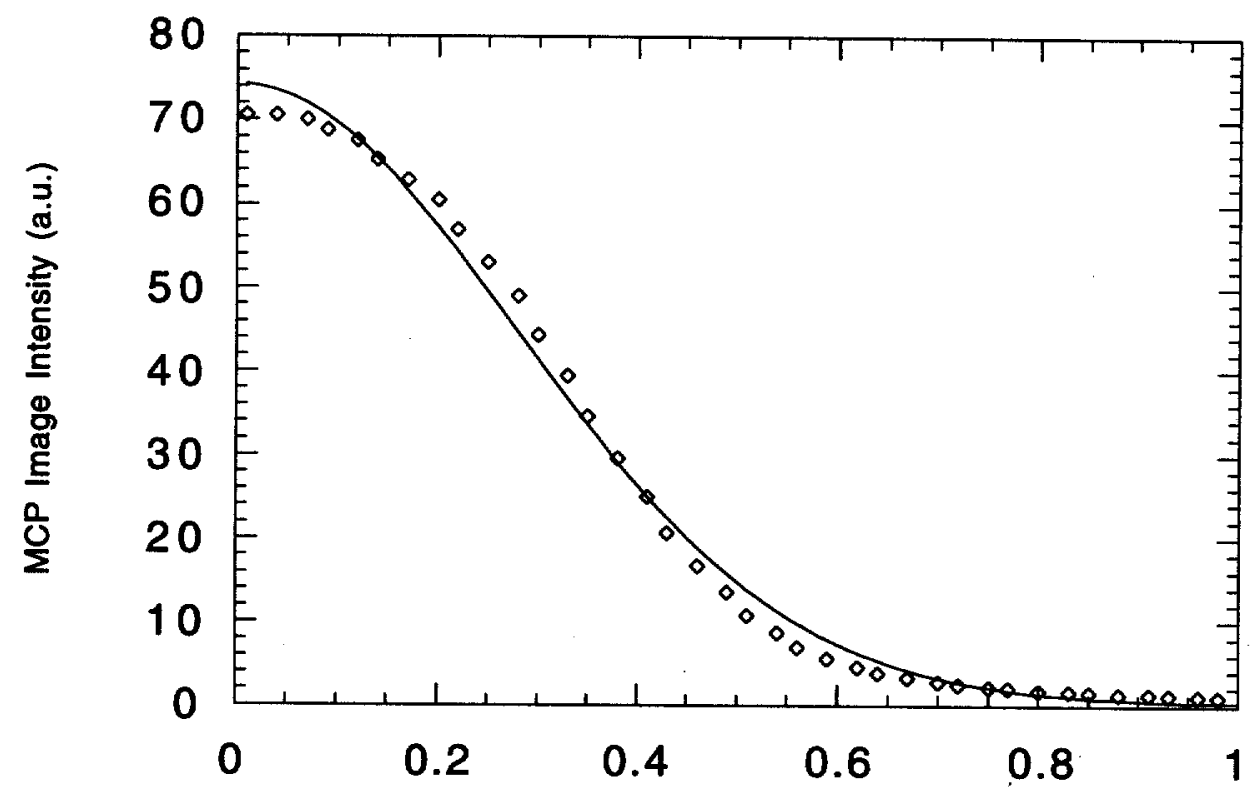

Radius (mm)

Figure 4.2 Radial electron density profile. $B=2 k G, L_{0}=18 \mathrm{~cm}, \mathrm{~N}=2 \times 106$, $\mathrm{t}=140 \mathrm{~ms}$. Curve is a gaussian fit, $\mathrm{n}_{\mathrm{o}}=74.1 \pm 7, \mathrm{r}_{\mathrm{p}}=0.391 \pm 4 \mathrm{~mm}$. This is representative of the most significant deviations from a gaussian profile.

The general thermal equilibrium distribution is [41],

$$
n(r)=n_{0} \exp \left[(e / k T) \varphi(r)-\infty r^{2}\right],
$$

where $\alpha$ is an arbitrary constant set by the initial angular momentum and energy of the system. In cases where the space charge potential is small, the equilibrium distribution would be similar to what is observed. Also, the bounce-averaged vacuum potential from electrode fringe fields increases quadratically with radius, producing a linearly-increasing field that mimics a uniform density distribution. When these external fields dominate $\varphi(r)$, they 
drive a constant-frequency equilibrium $\boldsymbol{E} \times \boldsymbol{B}$ rotation regardless of the actual distribution.

\subsubsection{Temperature}

The measurement of axial plasma temperature is performed as discussed in Sec. 2.5.4, but it is impractical to measure the temperature for every lifetime case. The temperature is assumed to follow the cyclotron cooling rate,

$$
\frac{d T}{d t}=-\frac{T}{\tau_{r}} R\left(\frac{\hbar \Omega}{k T}, \frac{\hbar \Omega}{k T_{w}}\right)+P_{E X},
$$

where

$$
\tau_{r} \equiv \frac{9 m c^{3}}{8 e^{2} \Omega^{2}} \approx \frac{4 \times 10^{8}}{B^{2}} \mathrm{~s}
$$

is the radiation time for a single electron in free space, and

$$
R(x, y)=x \frac{\exp (y)-\exp (x)}{[\exp (y)-1][\exp (x)-1]}
$$

is the correction factor for finite wall temperature $T_{w}$ and quantum effects [36]. $P_{e x}$ is an experimentally determined heating power, e.g. from electronic noise on the confining electrodes. This theory 
assumes the radial and axial motion is in equilibrium, and does not take into account plasma opacity or waveguide effects. For example, the cyclotron radiation is below the cutoff frequency for a $1 \mathrm{~cm}$ radius waveguide when $B<3.1 \mathrm{kG}$.

Figure 4.3 shows the early temperature evolution at $B=30 \mathrm{kG}$. The cooling matches the predicted rate quite well. The cooling at $B=60 \mathrm{kG}$ is shown in Fig. 4.4. The rapid cooling time of $79 \mathrm{~ms}$, instead of the predicted $110 \mathrm{~ms}$, would be expected in a $70 \mathrm{kG}$ field. The magnitude of the error is similar to UCSD measurements at $60 \mathrm{kG}$, but in the opposite direction. Noise and other effects would be expected to increase the cooling time. Background gas pressure or the collision cross section would have to be increased by 2-3 orders of magnitude to cause such rapid cooling, and the electrons are below the energy thresholds for most such interactions.

One possible explanation is resonant cooling. According to O'Neil [42], a collection of electrons radiating in a cavity can experience enhanced cooling if the cyclotron frequency is resonant with a cavity mode. The enhancement is maximized when the spread in cyclotron 


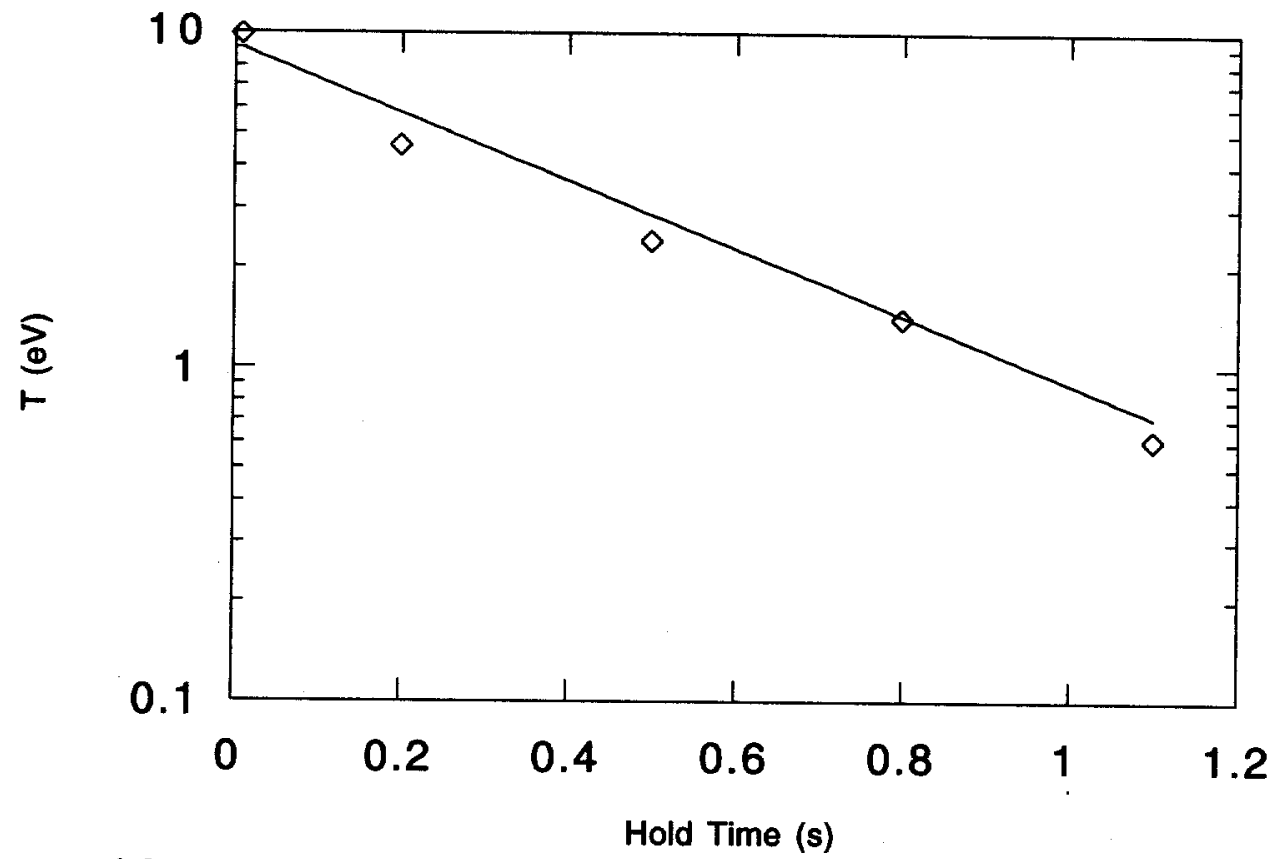

Figure 4.3 Cyclotron cooling at $B=30 \mathrm{kG}$. Curve is the predicted cooling rate, $\tau=0.43$. The cathode is biased to $-40 \mathrm{~V}$.

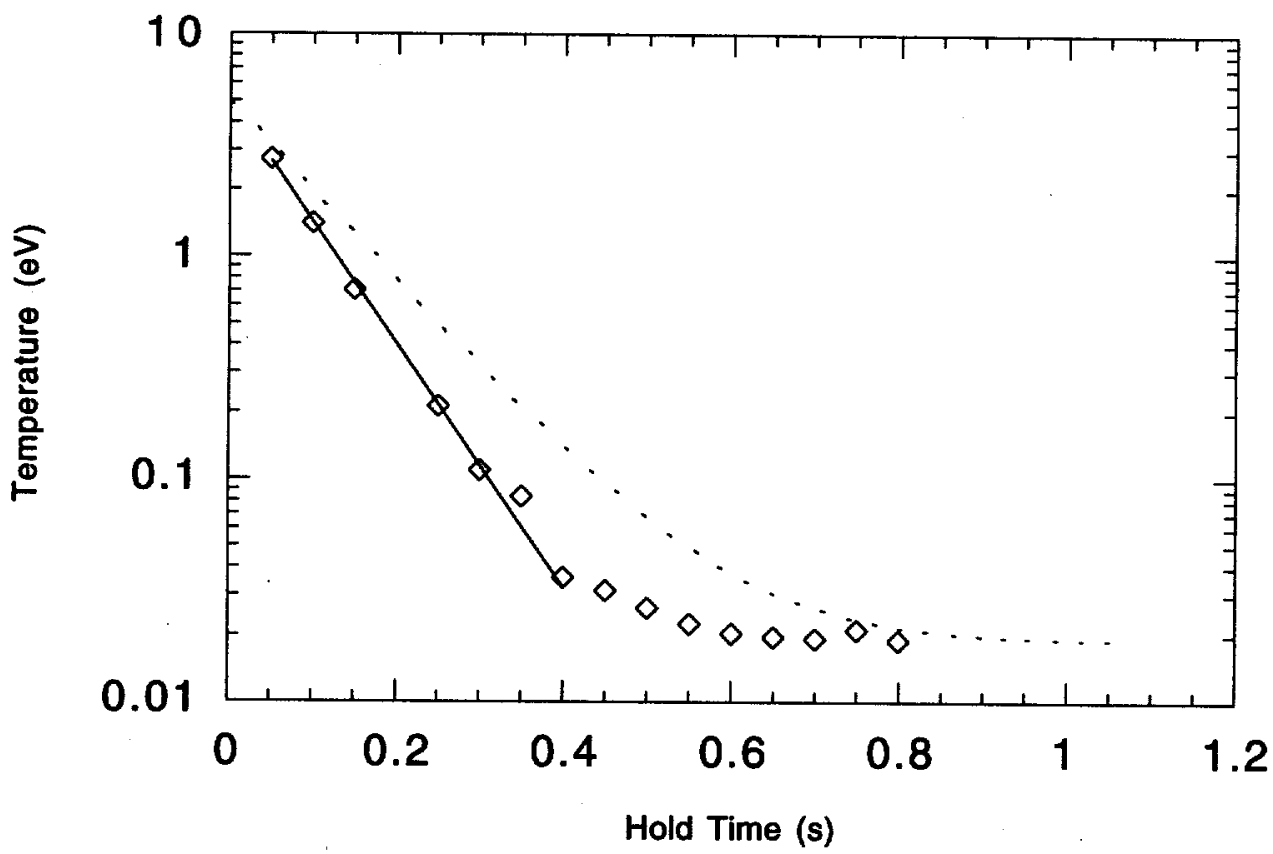

Figure 4.4 Cyclotron cooling in $60 \mathrm{kG}$ field. Solid curve is an exponential fit to the section $t \leq 0.4 \mathrm{~s}$, and gives a radiation time $\tau_{\mathrm{r}}=79 \pm 2 \mathrm{~ms}$. Dashed curve is the prediction with heating power $P_{e x}=0.1 \mathrm{eV} / \mathrm{s}$. Cathode is biased to $-10 \mathrm{~V}$. 
frequency $\Delta \Omega$ is comparable in magnitude to the linewidth of the resonance $\Gamma$ and the damping rate in the plasma $v_{p}$, at which point the enhancement approaches the $Q$ of the resonance, which can be large. The longitudinal field variation in this device results in $\Delta \Omega \leq 10^{-3} \Omega$. A gold or copper cavity has $\Gamma \approx 10^{-4} \Omega$, so the $\Delta \Omega \approx \Gamma$ criterion is satisfied for all $B$. The enhancement is far from maximized, because $v_{p}(\Omega) \approx f(\Omega) \omega_{p}{ }^{2}$ is several orders of magnitude larger than the other parameters, even at $B=60 \mathrm{kG}$. The function $f(\Omega)$ is the normalized distribution of cyclotron frequencies in the plasma, weighted by the relative field strength. The relationship $\Delta \Omega \approx \Gamma \ll v_{p}$ could explain why we see a slight enhancement only at the highest field strength. This assumes that a resonance exists, and the present assembly is more appropriately described as a waveguide, with a continuum of normal modes, rather than a resonant cavity.

The low-temperature measurements shown are made with a resistor placed in series with the dump power supply. In combination with the cable capacitance this acts as a low-pass filter. With the resistor 
removed, as is necessary for rapid voltage switching, the temperature levels off near $0.03 \mathrm{eV}$ instead of $0.02 \mathrm{eV}$, an increase in $P_{\text {ex }}$ of nearly a factor of 2 . This suggests that a second resistor in series with the injection supply should eliminate much of the remaining noise if colder temperatures are required. Relays could be used to short out the filters during voltage switching.

\subsubsection{Density Measurement}

The peak density is determined from the radial profile and total charge signal from the MCP, in combination with the calculated length. Because of the dependence of length on the temperature, there is the potential for systematic errors based on the assumed value of $P_{e x}$ and the actual initial energy. The error is at most a few percent in the longer electrodes because the cooling contraction $\Delta L / L_{0} \ll 1$, and this is a small error in the calculation of $\Delta L$. In short electrodes $\left(L_{0}<8 \mathrm{~cm}\right)$ the errors may be over $10 \%$.

As discussed in Sec. 2.5.3, the frequency of the diocotron mode is a commonly-used calibration of the total density. Two significant 
problems arise in the use of this technique when the plasma radius is small. First, the perturbation expansion used by Prasad and O'Neil [43] to correct for finite-length effects breaks down. The first-order correction,

$$
\omega_{d}^{(1)}=f\left(\frac{r_{p}}{L}\right)\left(\omega_{r}-\omega_{d}^{(0)}\right) \frac{r_{p}}{L}
$$

becomes large relative to the zero-order term when

$$
\frac{\omega_{r}}{\omega_{\mathrm{d}}^{(0)}} \propto \frac{n}{N /\left(L R_{w}^{2}\right)}=\frac{R_{w}^{2}}{r_{p}^{2}}>1
$$

as is the case in our device.

The second and more significant weakness in the classic diocotron calculation is that it ignores the effect of the end potentials. The barriers are assumed to be sharp and infinite, determining only the length of the plasma. In practice, there are substantial radial electric fields present in the fringe-field region near the barrier electrodes. Because $\omega_{d} \propto N / L$, these external fields will dominate the field caused by even a high-density off-axis plasma if $r_{p}$, and therefore $N$, is small. In this case, the orbiting motion is more properly attributed to 


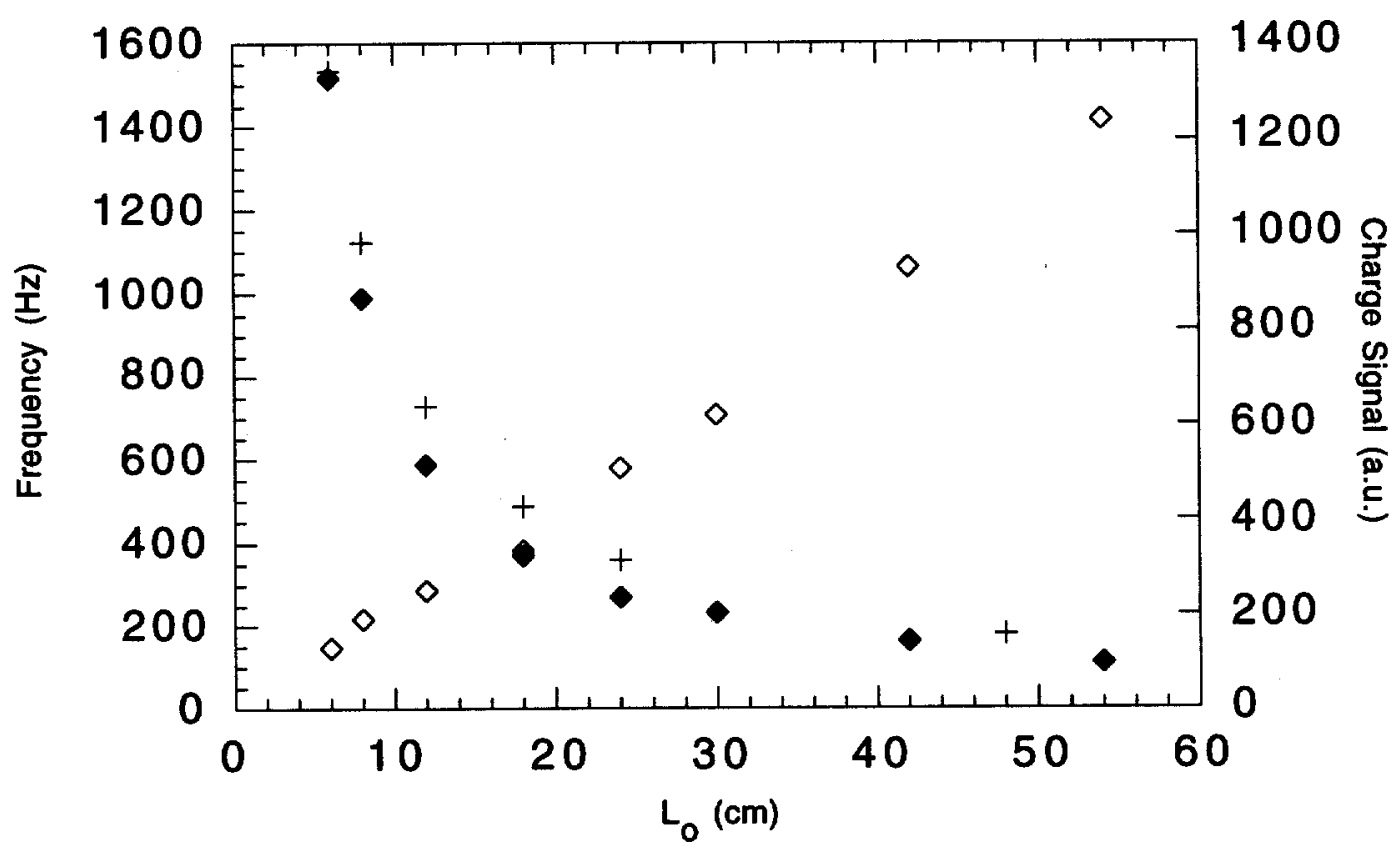

Figure 4.5 Measured "diocotron" frequency vs. electrode length. The total charge $(0)$ increases linearly, but $v_{d}(\diamond)$ varies like $L^{-1}$, as expected for magnetron drift $(+)$. Measured $n=3.5 \times 10^{7} \mathrm{~cm}^{-3}, r_{p}=0.1 \mathrm{~mm}$, implies $v_{d}=1.68 \mathrm{~Hz}$.

magnetron drift, rather than a diocotron mode.

Fig. 4.5 compares the measured "diocotron" frequency to the expected magnetron drift for a range of $L_{0}$. The drift rate is calculated for a particle with $W_{\|}=3.75 \mathrm{eV}\left(5 \mathrm{eV}, 30^{\circ}\right.$ pitch angle), using the single-particle ray tracing calculations discussed in Appendix C. The small discrepancies can be attributed to the difference between the actual and assumed values of $W_{\|}$. Lower 
energy particles penetrate less deeply into the fringe fields, experiencing a lower bounce-averaged $E_{r}$. Note that the measured orbit frequencies would correspond to $n \approx 3 \times 10^{9} \mathrm{~cm}^{-3}$ when $r_{p}=0.1 \mathrm{~cm}$, the largest electron radius studied and the target radius of the positron plasma. Calibration of the charge collection in this case indicates $n=3.5 \times 10^{7} \mathrm{~cm}^{-3}$. Clearly, the diocotron motion cannot be used as a density diagnostic for these plasmas.

\subsubsection{Lifetime Calculation}

The standard parameter for characterizing the confinement of nonneutral plasmas is the "mobility" time: the time required for the central density to fall to one-half of its initial value, $\tau_{m}$ [44]. This allows a general comparison of confinement across a wide range of parameters when the details of the evolution can vary significantly. In the following studies, the data is first analyzed by this technique for direct comparison to previous work.

In addition, a method has been developed to characterize the instantaneous evolution of the plasma. Assuming thermal 


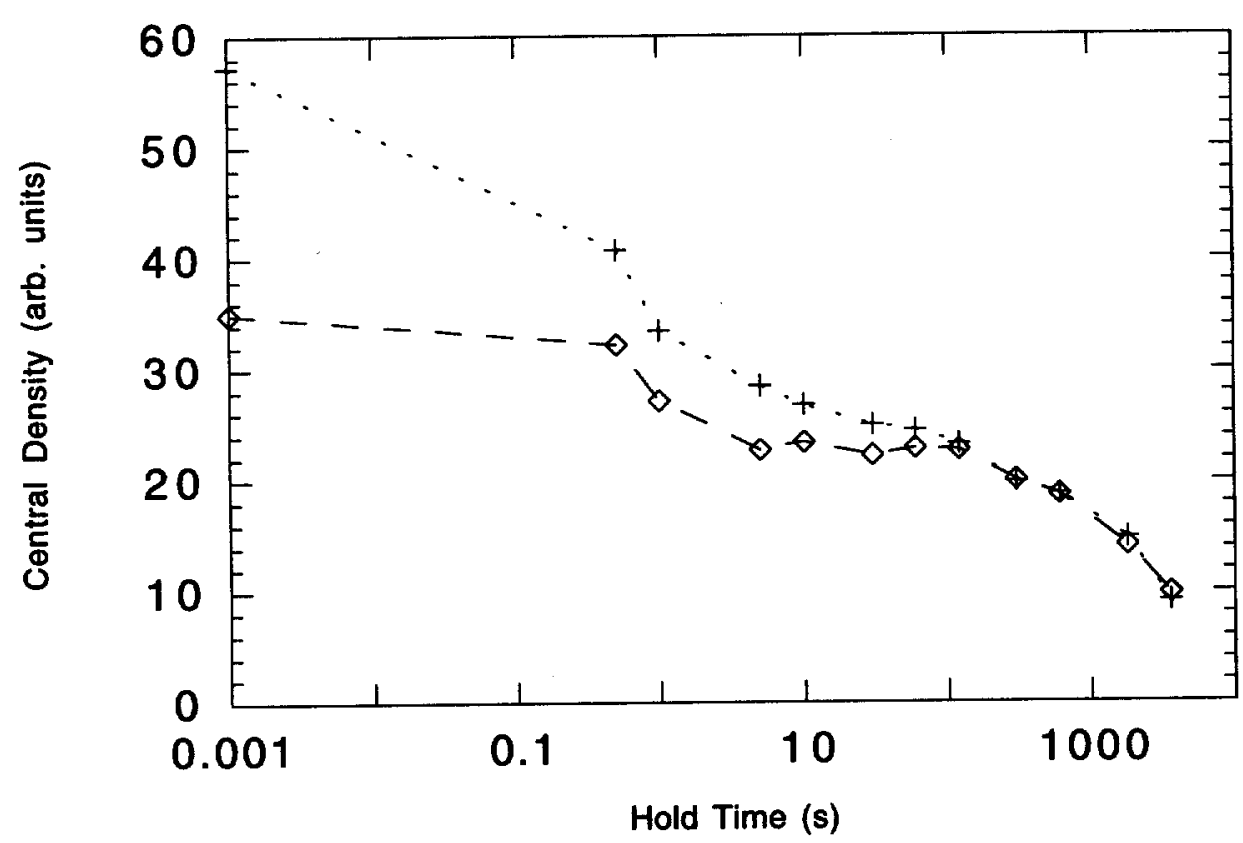

Figure 4.6 Comparison of plasma evolution in $2 \mathrm{~cm}$ electrodes. The evolution is qualitatively similar, but the half-life $\tau_{\mathrm{m}}$ changes from $5 \mathrm{~s}(+)$ to $940 \mathrm{~s}(0)$.

equilibrium, a plasma of given temperature, density, and radius should have a characteristic evolution independent of its prior history. Fig. 4.6 compares the evolution of two plasmas confined in different $2 \mathrm{~cm}$ electrodes. The initial density in the two cases varies by less than a factor of 2 while $\tau_{m}$ changes drastically, from $5 \mathrm{~s}$ for the high density case to $940 \mathrm{~s}$ at lower density. Even ignoring the first data point as a nonequilibrium state, $\tau_{m}=290 \mathrm{~s}$ vs. $1300 \mathrm{~s}$. The density ratio at $0.5 \mathrm{~s}$ is 1.25 , while the lifetime ratio is 4.5 . Qualitatively, the two evolutions are clearly similar, and once the 
densities are matched further evolution is identical.

The addition of cyclotron cooling also adds a significant timedependent factor to the plasma evolution. Both the temperature and the length of the plasma change as it cools. In the shortest electrodes at low density, the length can change by a factor of 4 in the first second of the confinement period. It is therefore reasonable to use an alternative to the time-independent parameter $\tau_{m}$.

In this study, the time-dependent evolution is characterized by the instantaneous exponential lifetime,

$$
\tau_{i}=-n\left(\frac{d n}{d t}\right)^{-1}
$$

If the decay rate is proportional to the density, then the evolution will be an exponential and $\tau_{i}$ will be constant during the evolution, with a value $\tau_{i}=\tau_{m} / \ln (2)$. If, as predicted by the UCSD experiments, the lifetime improves as density declines, then $\tau_{i}$ should increase as a function of time, and the evolution curve plotted on a semilogarithmic scale will be concave upward. Fig. 4.7 shows examples of 

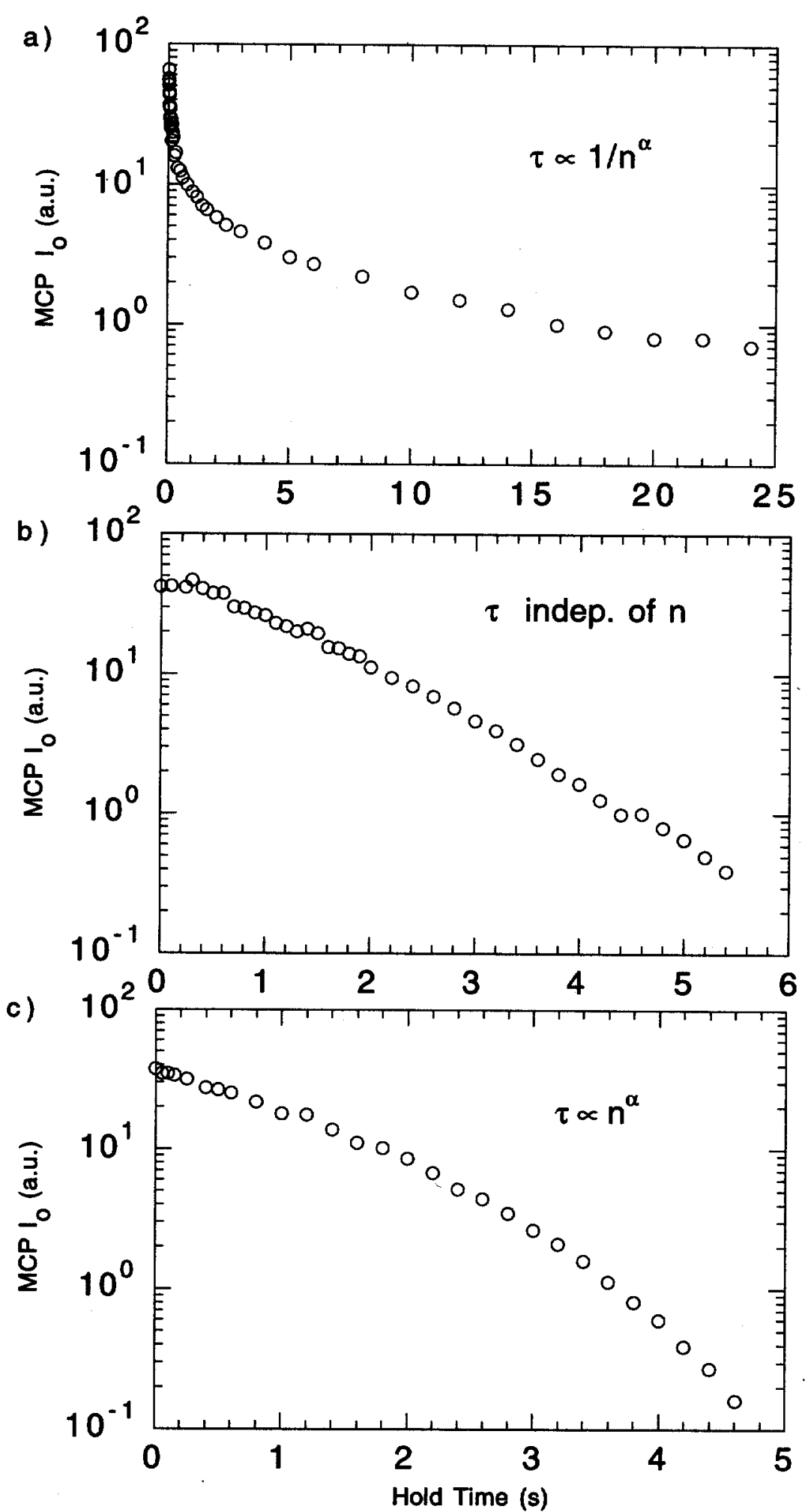

Figure 4.7 Plasma lifetime evolution: a) improving, b) constant, and c) decreasing in time. $L_{0}=18 \mathrm{~cm}$. 
both of these cases, as well as a situation in which the confinement apparently degrades with time.

The challenge in this analysis is the accurate calculation of the instantaneous slope. Fitting the entire curve is difficult given the variety of shapes found in different parameter regimes. Forward differencing of the raw data results in considerable scatter. Instead, the data is smoothed by fitting short segments to an exponential. One advantage of this technique is that the fit provides a direct value and error estimate for $\tau_{i}$. Fig. 4.8 shows $\tau_{i}$ as calculated using 3 and 5

points for the plasma evolution of Fig. 4.7a. The three-point technique shows greater scatter, but the five-point technique misses rapid changes. In general, the five-point lifetime was found sufficient to capture the dynamics of the evolution, except in those cases where the number of data points was $<10$. 

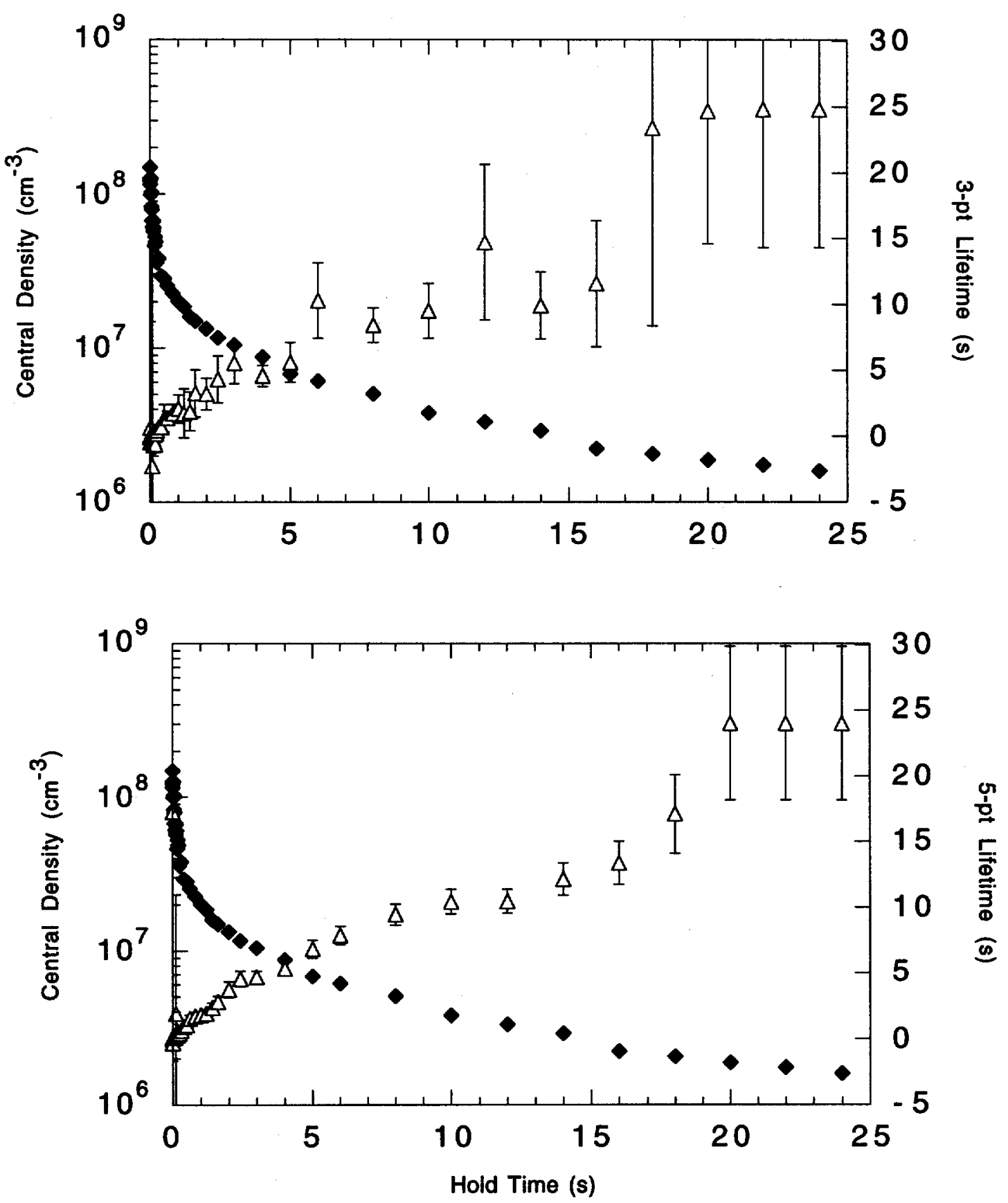

Figure 4.8 Instantaneous lifetime calculation. An exponential is fit to every a) 3 or b) 5 points. Five-point fit appears to capture the dynamics with less scatter. 


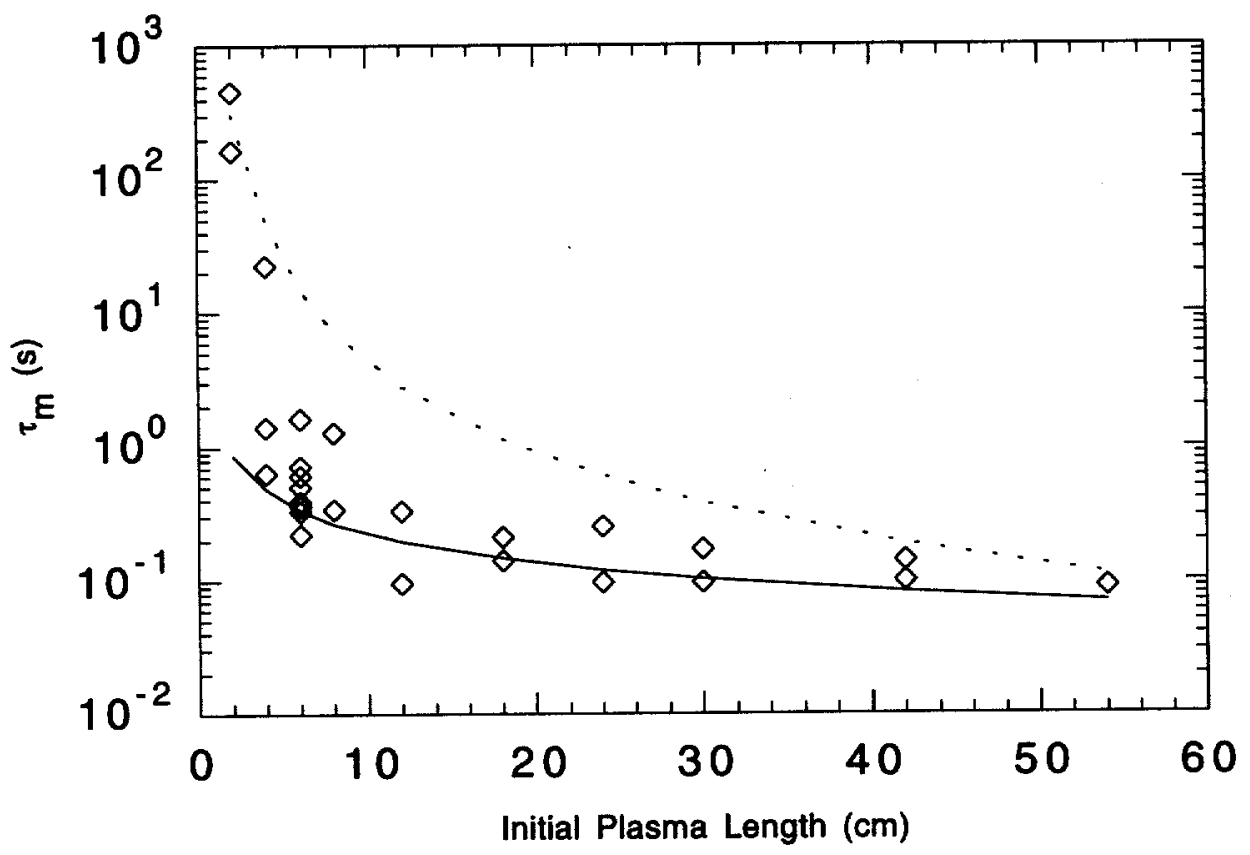

Figure 4.9 Length dependence of $\tau_{\mathrm{m}} . \mathrm{B}=30 \mathrm{kG}, \mathrm{n}_{\mathrm{o}}=3.8 \times 10^{7} \mathrm{~cm}^{-3}$. Solid curve is a power law fit, $\alpha=-0.64 \pm 0.03$. Dashed curve is $L^{-2}$.

\subsection{Half-Life Analysis}

\subsubsection{Length}

The predicted length dependence according to the empirical scaling is $\tau_{m} \propto L^{-2}$. Fig. 4.9 shows $\tau_{m}$ as a function of length at $B=3 \mathrm{~T}$, $n=1 \times 10^{8} \mathrm{~cm}^{-3}$. A fit to a power law gives $\tau_{m} L^{-0.64 \pm 0.03}$. By inspection, there is little apparent dependence on length above $8 \mathrm{~cm}$, while below that point the dependence becomes very strong. Adjusting the 
weighting in the fit to emphasize the short lengths gives $\tau_{m} \propto L^{-5.8 \pm 1.3}$.

As in previous studies, there is considerable reproducible scatter in the lifetimes in different short electrodes. This can be accounted for by variations in the alignment. It has been shown [35] that alignment differences as small as $10^{-4}$ radians can have a significant impact on the evolution. For a $6 \mathrm{~cm}$ electrode, this corresponds to a displacement of one end by only $6 \mu \mathrm{m}$, well within the mechanical tolerance of the trap design.

Even with this scatter, the differences between the current data and the expected values are marked. Based on the scaling laws used to design this device, the lifetime would be expected to change by more than a factor of 4 between the $24 \mathrm{~cm}$ and $52 \mathrm{~cm}$ electrodes, and no significant change is seen.

\subsubsection{Magnetic Field}

As with length, the magnetic field dependence of the confinement was found to be less significant than predicted. Fig. 4.10 shows the 


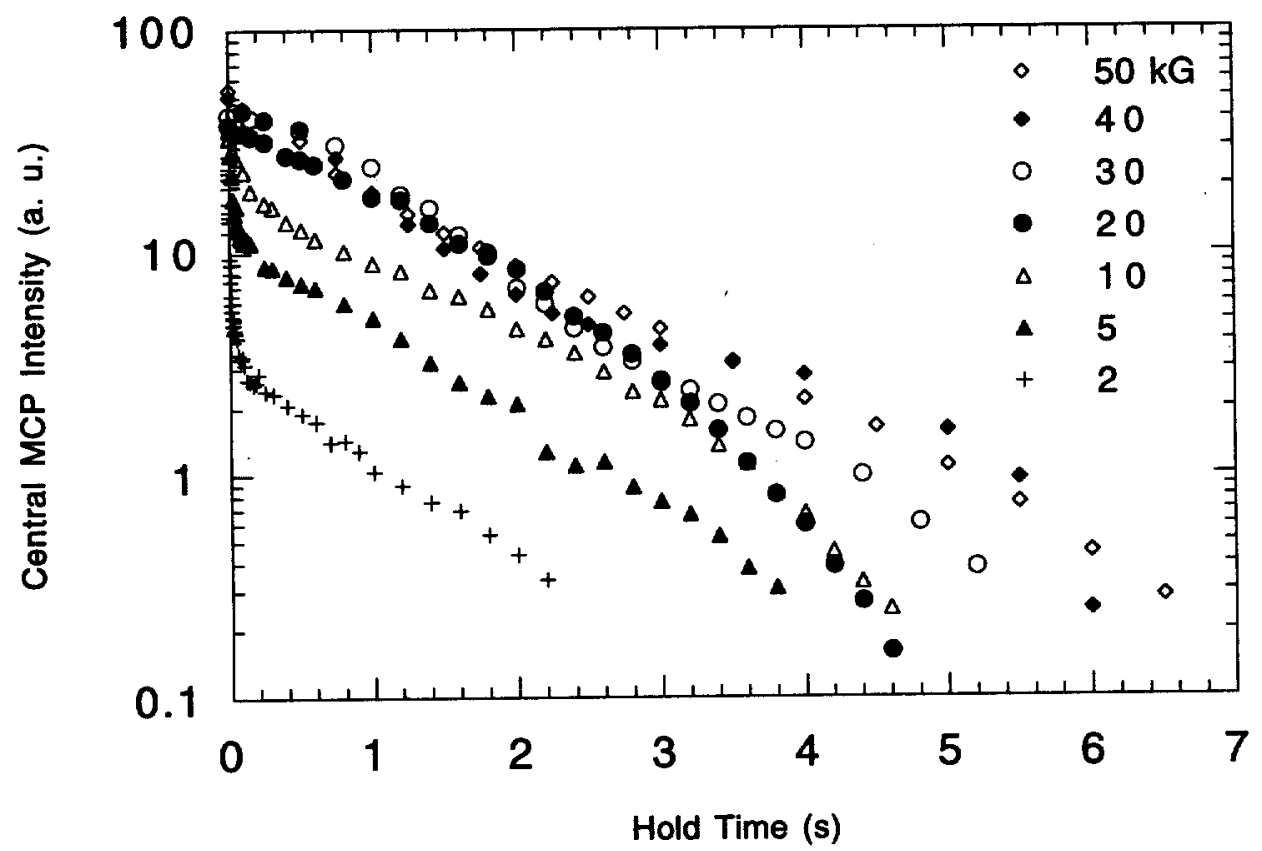

Figure 4.10 Evolution of electron density for $B=2-50 \mathrm{kG}$. At short times, the low-field plasmas evolve much more rapidly, but the long-time evolution is remarkably similar at all fields.

density evolution as a function of time for $B=2-50 \mathrm{kG}, L_{0}=18 \mathrm{~cm}$, and $n_{0}=10^{8} \mathrm{~cm}^{-3}$. Fig. 4.11 shows the corresponding $\tau_{m}$ vs. $B$. The strong dependence for $B<2 \mathrm{kG}$ is in rough agreement with the prediction, but at higher fields the improvement is much less than expected. If one measures $\tau_{m}$ beginning at $t=0.1 \mathrm{~s}$, there is no field dependence at all.

This latter comparison is a bit misleading, since differences in early evolution lead to a different "initial" density; however, the 


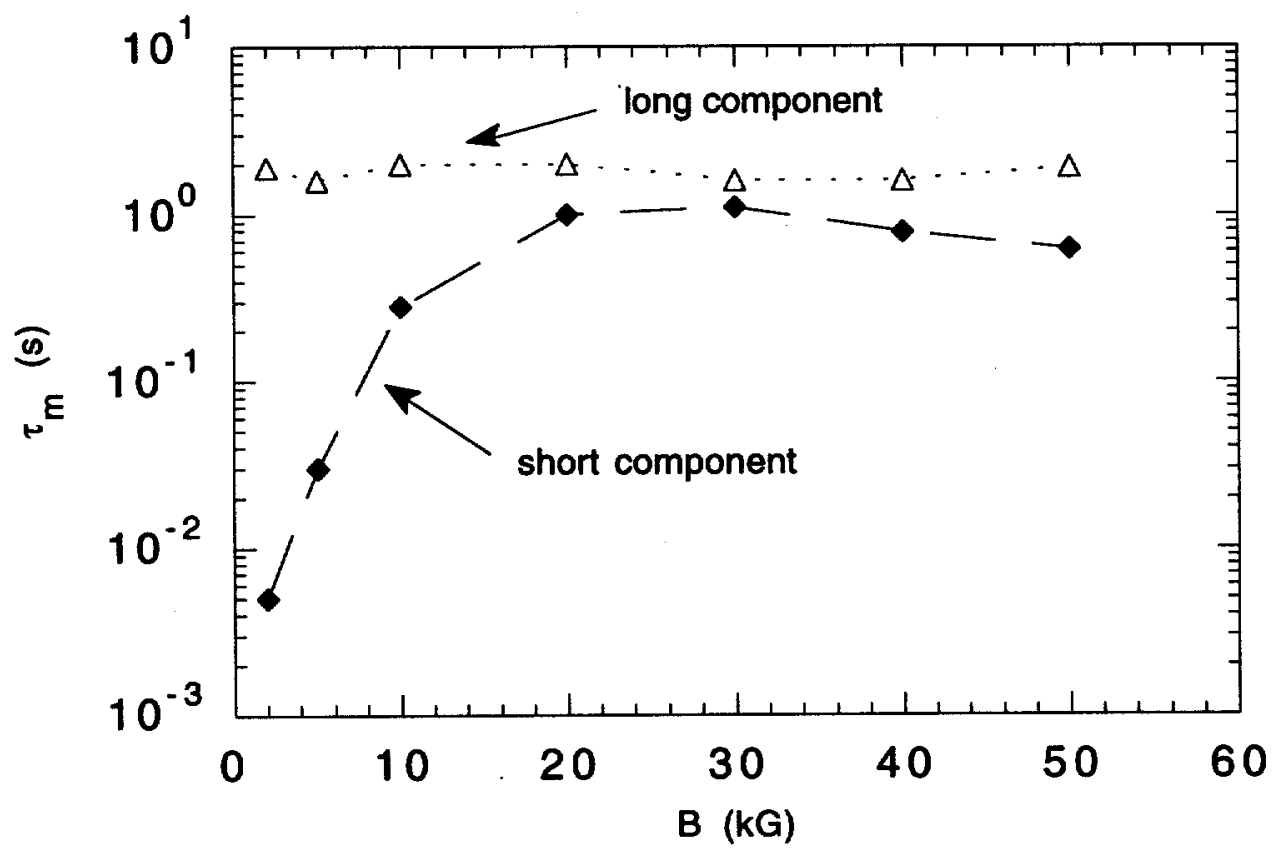

Figure 4.11 Magnetic field dependence of $\tau_{\mathrm{m}}$, as measured from $\mathrm{t}=1 \mathrm{~ms}(\bullet)$ and $\mathrm{t}=0.1 \mathrm{~s}(\Delta) . \mathrm{L}_{0}=18 \mathrm{~cm}$. $\mathrm{n}_{0} \approx 6 \times 10^{8}$, decreasing to about $1 \times 10^{8}$ below $5 \mathrm{kG}$.

instantaneous lifetime data discussed below does clearly

demonstrate that plasmas with widely varying lengths and magnetic fields all evolve to states with very similar lifetimes.

\subsubsection{Density}

The most striking disagreement between the present study and previous work is in the confinement dependence on density. For the parameter range of this device, the density scaling differs qualitatively from the expected behavior. Instead of changing inversely with the density, in most cases the lifetime improves as the 
density increases. Figs. 4.12 and 4.13 show the effect of density on $\tau_{m}$ at $B=30 \mathrm{kG}$ in 6,18 and $24 \mathrm{~cm}$ electrodes. At low densities, $\tau_{m}$ increases rapidly, then levels off and decreases at high density. The difference in $\tau_{m}$ in the two long electrodes at higher density, suggesting a reactivation of the length dependence, appears to be an artifact of the variations in the shape of the evolution. No length dependence is observed in the long electrodes. At $B=540 \mathrm{G}$, comparable to the field strength in UCSD work, the inverse-density relationship is seen (Fig. 4.14), although it slower than the predicted $\tau_{\mathrm{m}} \propto 1 / \mathrm{n}^{1-2}$

\subsubsection{Radius}

The study of radial dependence is possible in this device because of the large range of radii available. In most nonneutral devices, $R / r_{p} \approx 2$, while in this case $R / r_{p}=10-100$, giving a much greater dynamic range. 


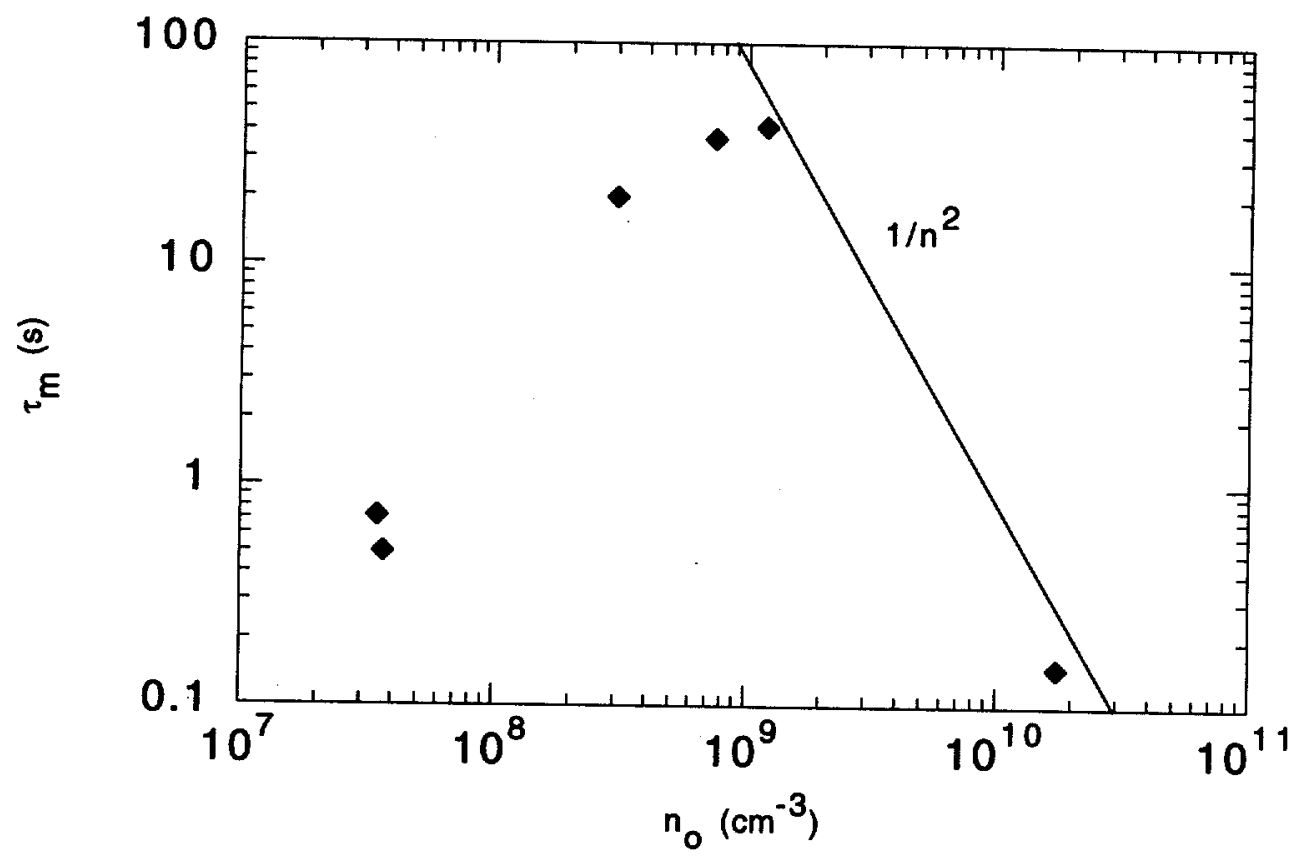

Figure 4.12 Density dependence of the half-life in a $6 \mathrm{~cm}$ electrode. $\mathrm{B}=30 \mathrm{kG}$, $r_{0}=0.1 \pm 0.01 \mathrm{~mm}$. Solid line is $\tau_{\mathrm{m}} \propto \mathrm{n}-2$, for comparison.

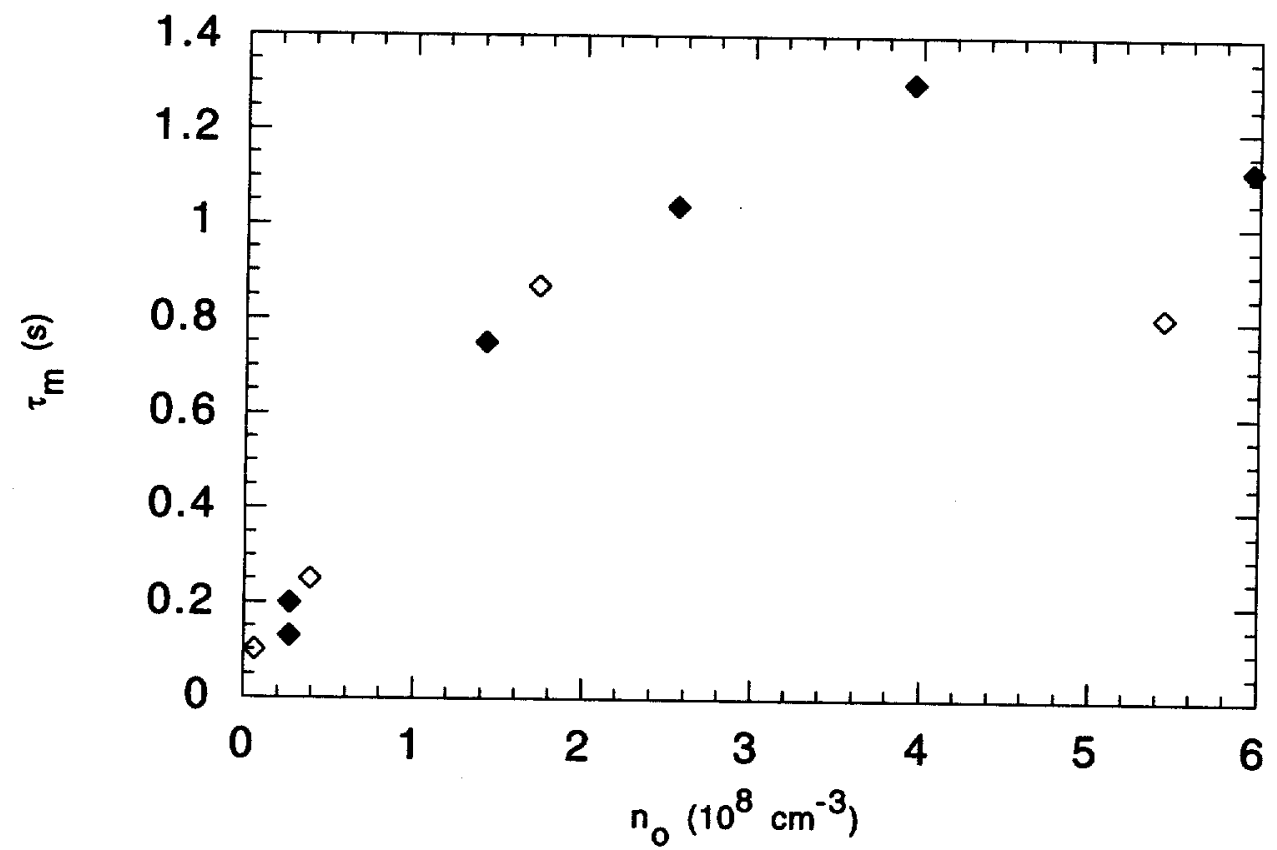

Figure 4.13 Density dependence of the half-life in $18 \mathrm{~cm}(\bullet)$ and $24 \mathrm{~cm}(\vartheta)$ electrodes. $B=30 \mathrm{kG}, \mathrm{r}_{0}=0.1 \pm 0.01 \mathrm{~mm}$. 


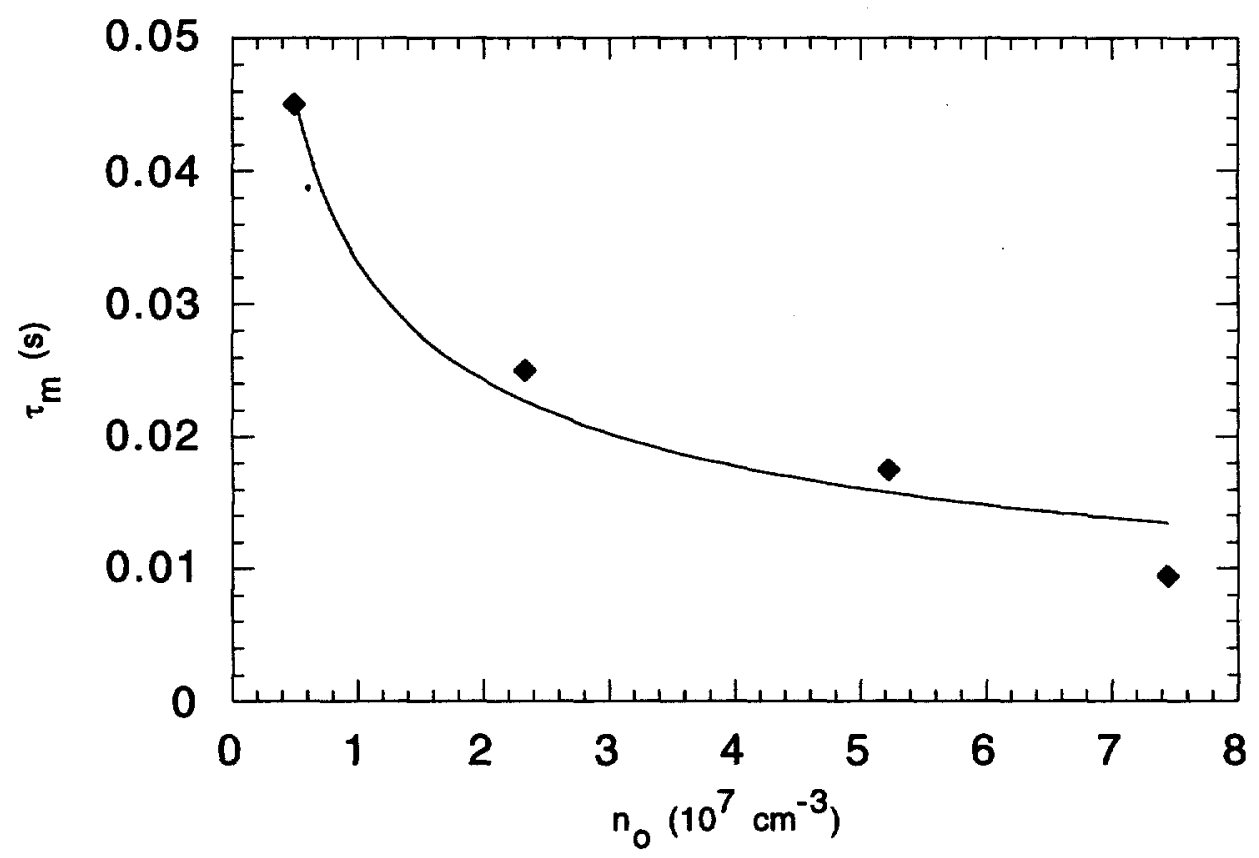

Figure 4.14 Low-field density dependence of half-life in $18 \mathrm{~cm}$ electrode. $\mathrm{B}=540 \mathrm{G}, \mathrm{r}_{0}=0.19 \pm 0.01 \mathrm{~mm}$. Curve is $\tau_{\mathrm{m}} \propto \mathrm{n}^{-1 / 2}$, for comparison.

Fig. 4.15 shows the improved confinement with increased initial radius, $L_{0}=18 \mathrm{~cm}, B=30 \mathrm{kG}$. The strong radial dependence $\tau_{m} \propto r_{p}{ }^{1.7 \pm 0.2}$ is inconsistent with bounce resonance theory. The rotation frequency is nominally dependent only on the central density. The gaussian profile of these plasmas does introduce shear in the rotational motion, but this does not explain the observations. Larger radius plasmas have a broader range of rotational frequencies, and thus a higher probability that some portion of the charge distribution is resonant with a field error. Larger plasmas should experience 


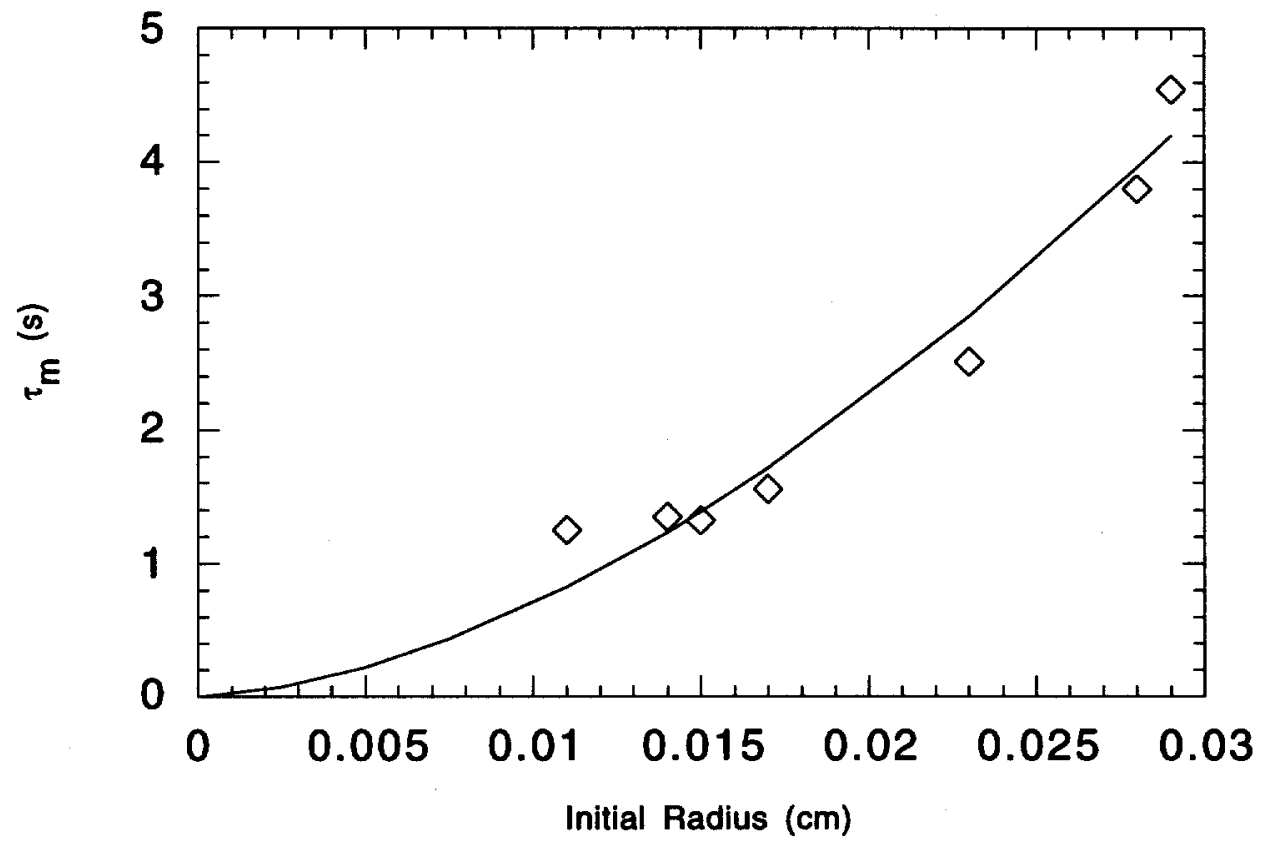

Figure 4.15 Radial dependence of $\tau_{\mathrm{m}} \cdot \mathrm{L}_{0}=18 \mathrm{~cm}, \mathrm{~B}=30 \mathrm{kG}, \mathrm{n}_{0}=3 \times 10^{8} \mathrm{~cm}^{-3}$. Curve is a fit to a power law, $\alpha=1.7 \pm 0.2$.

enhanced transport, while the opposite effect is observed.

\subsubsection{Comparison to $(B / L)^{2}$ and Bounce-Resonance Theory}

Fig. 4.16a summarizes all the half-lifetimes measured on this device, plotting $\tau_{m}$ vs. $L / B$, and comparing to the confinement scaling exptrapolated from the UCSD devices $V^{\prime}$ and EV. Given the large differences in density and temperature between this device and the previous studies, the disparities are not surprising. Data from the UCSD high-field cryogenic device CV, shown in Fig. 4.16b, shows 

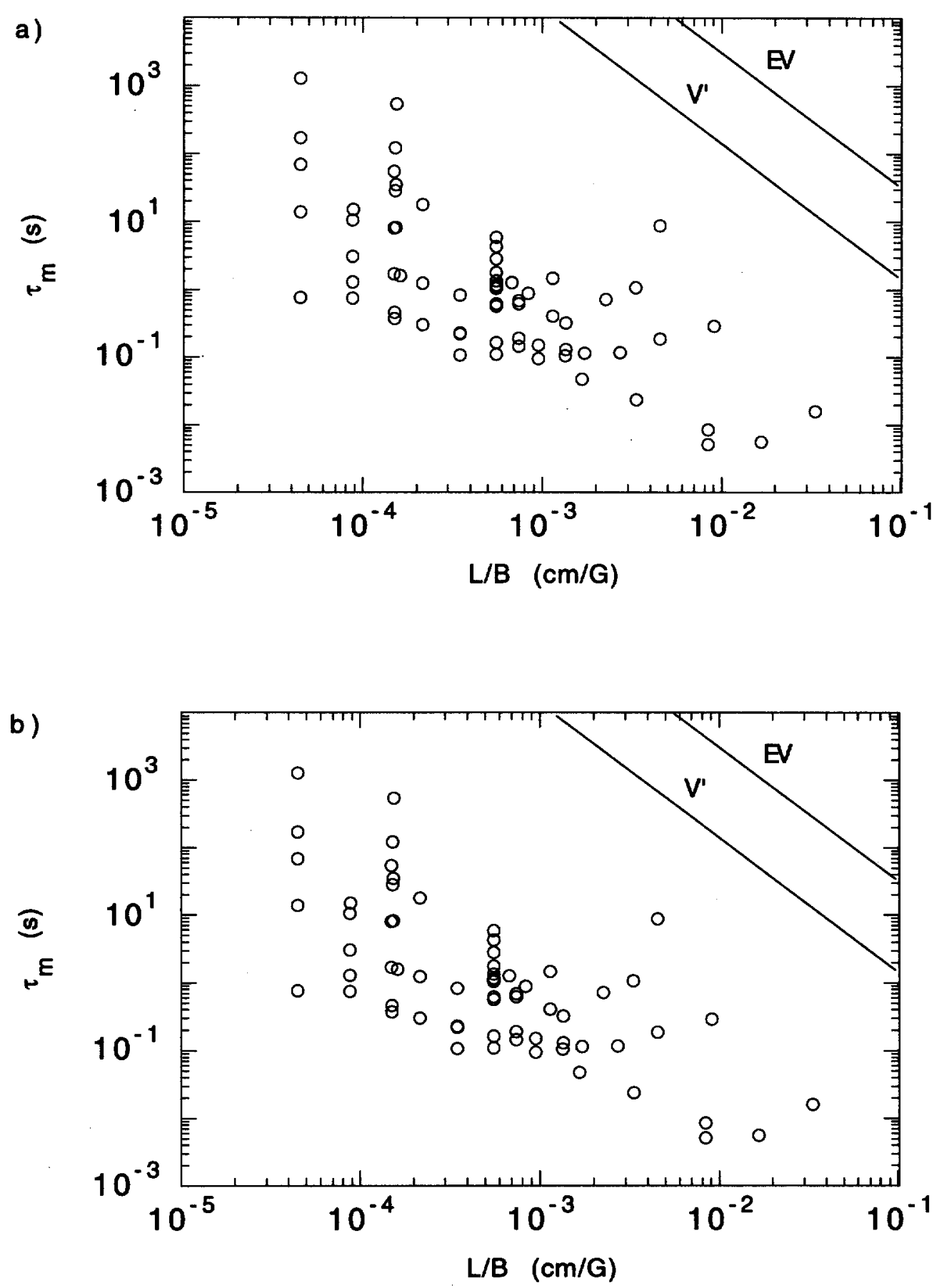

Figure 4.16 Comparison to UCSD L/B scaling. Solid lines are extrapolated from room-temperature devices. Data for the LLNL device (a) is similar to the cryogenic UCSD device (b - reproduced from Ref. 45, Fig. 3.5, with permission of the author). 
similar scatter [45]. Fig. $4.17 \mathrm{a}$ shows $\tau_{m}$ vs. $\omega_{\rho} / \omega_{b}$, the critical parameter in the bounce-resonance theory. (See Sec. 1.3 and Appendix A.) Fig. 4.17b shows the comparable data for CV. The bounce frequency $\omega_{b}$ is somewhat difficult to characterize for large $B$, because of the time-dependence of the temperature, and this could in principle account for some of the disparity. In $\mathrm{CV}$, plasma temperature is held constant by controlled oscillation of the confining voltages. The CV data are in better agreement with the bounceresonance scalings.

Analysis of LLNL data using the instantaneous lifetime $\tau_{i}$, shown in Fig. 4.18, provides no better agreement with the predictions, even though it accounts for changes in temperature. The scatter is enormous, indicating that the compound parameters $\omega_{b}$ and $\omega_{r}$ are not meaningful for characterizing the confinement of this device. The next section will demonstrate that there are some clear systematic trends hidden in the apparent scatter of Fig. 4.18 that may explain the difference between the LLNL and CV data in Fig. 4.17. 

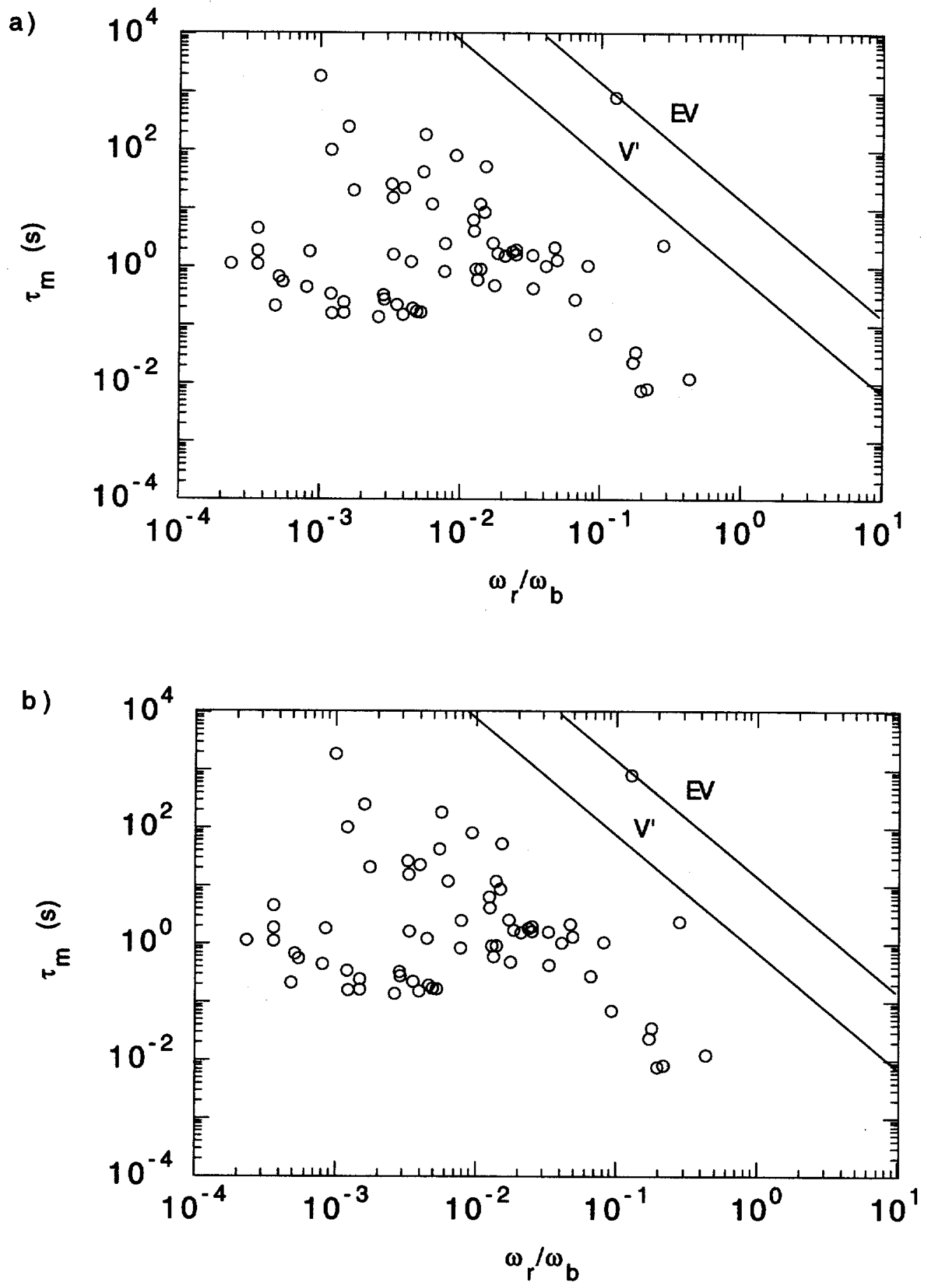

Figure 4.18 Comparison to UCSD bounce-resonance scaling, for LLNL (a) and UCSD CV (b) data. (b reproduced from Ref. 45, Fig. 3.6, with permission of the author.) 


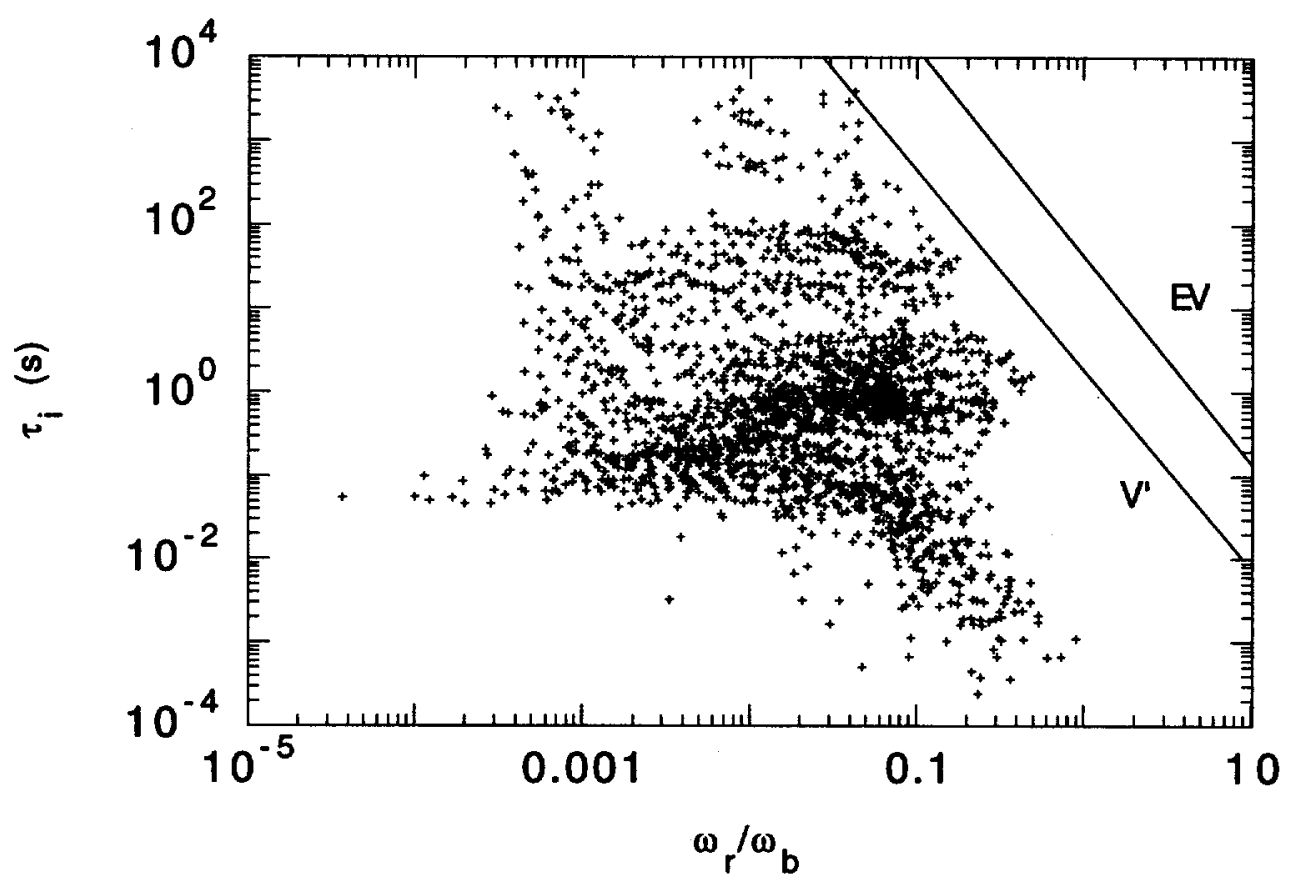

Figure 4.19 Comparison of the instantaneous lifetime to extrapolated UCSD bounce-resonance scalings.

\subsection{Dynamic Evolution Analysis}

The use of the instantaneous lifetime $\tau_{i}$ allows study of the dynamics of the plasma evolution. As discussed previously, the plasma evolution can be characterized by the relationship $\tau_{i} \propto n^{\alpha}$. The three regimes represent $\alpha<0, \alpha=0$, and $\alpha>0$. In a given evolution, these represent $\tau_{i}$ improving with time, constant, and degrading with time, respectively. The transition from one regime to another is 


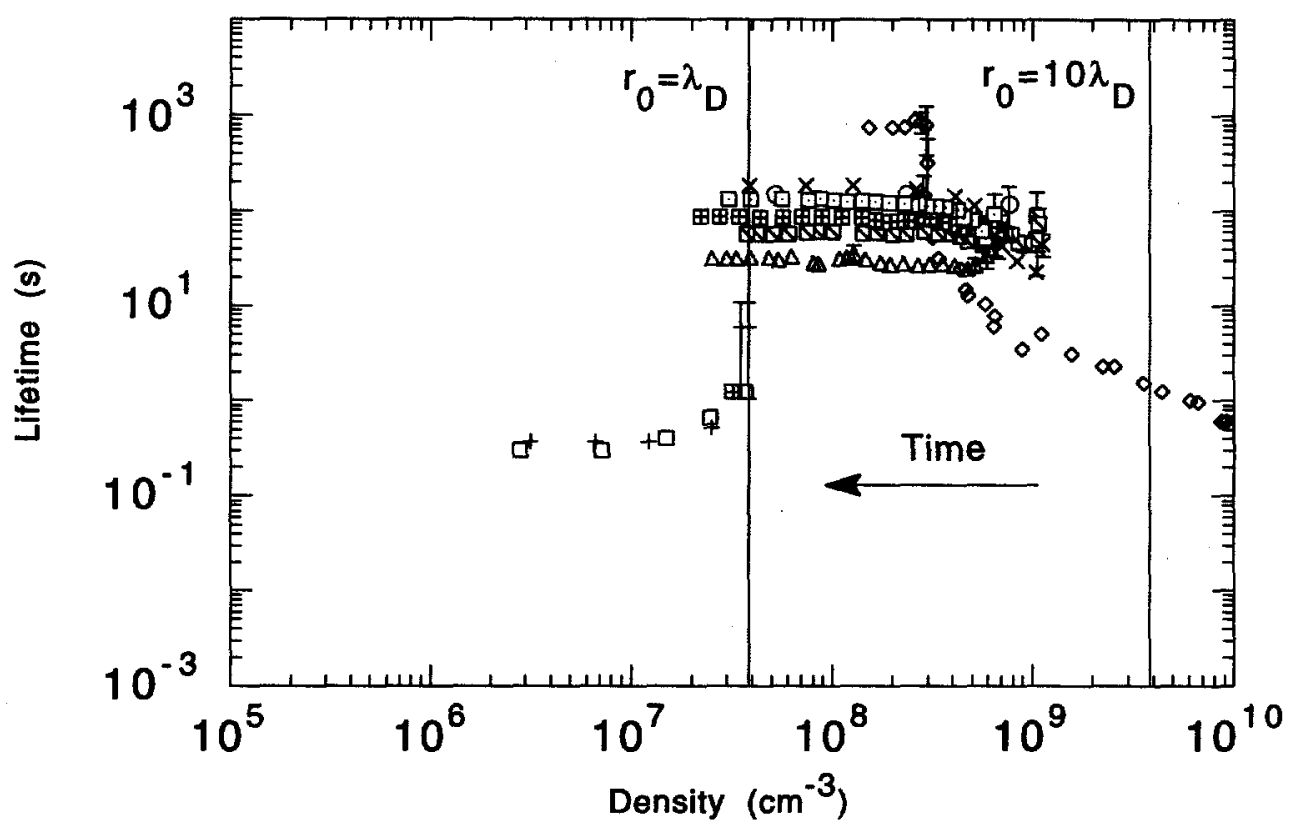

Figure 4.19 Lifetime evolution, $L_{0}=6 \mathrm{~cm}, B=30 \mathrm{kG}$. Each plot shape represents a separate data series, with hold time and radius increasing as density decreases. Vertical lines separate regions based on the importance of Debye shielding.

demonstrated clearly in Fig. 4.19 , showing $\tau_{i}$ vs. $n$ for all the data sets in $L_{0}=6 \mathrm{~cm}, B=30 \mathrm{kG}$. In this and the following figures, a single plasma evolution is traced moving from right to left, in the direction of decreasing $n$. Error bars are shown only if they are significantly larger than the plot character.

This plot provides significant insight into the importance of density 
in the high-field data. All electrodes $L_{o}>2 \mathrm{~cm}$. display a similar transition from an $\alpha>0$ regime to $\alpha=0$ when $n_{0} \approx 10^{8} \mathrm{~cm}^{-3}$. This is a significant initial density range because $\lambda_{D} / r_{p} \geq 1$, where plasma shielding of external fields becomes an issue. Since $r_{p}, \lambda_{D} \propto n^{-1 / 2}$ for constant $N$, the shielding ratio remains constant throughout the plasma evolution, as does $\tau_{i}$.

Fig. 4.20 shows the lifetime improvement in an $18 \mathrm{~cm}$ electrode as the radius is increased at constant density. The increase in $r_{p}$ improves the relative effectiveness of the shielding. Initially, when density is high and the radius is small, the radial dependence is in good agreement with the $\approx r^{2}$ dependence seen in the half-life data. At later times, (lower density, larger radius) the shape of the curve remains qualitatively similar, but the dependence steepens. This is perhaps due to a slight "dispersion" in the lifetimes. The lifetimes of the smallest plasmas decrease significantly, while the lifetimes of the larger, better-shielded plasmas remain roughly constant or even 


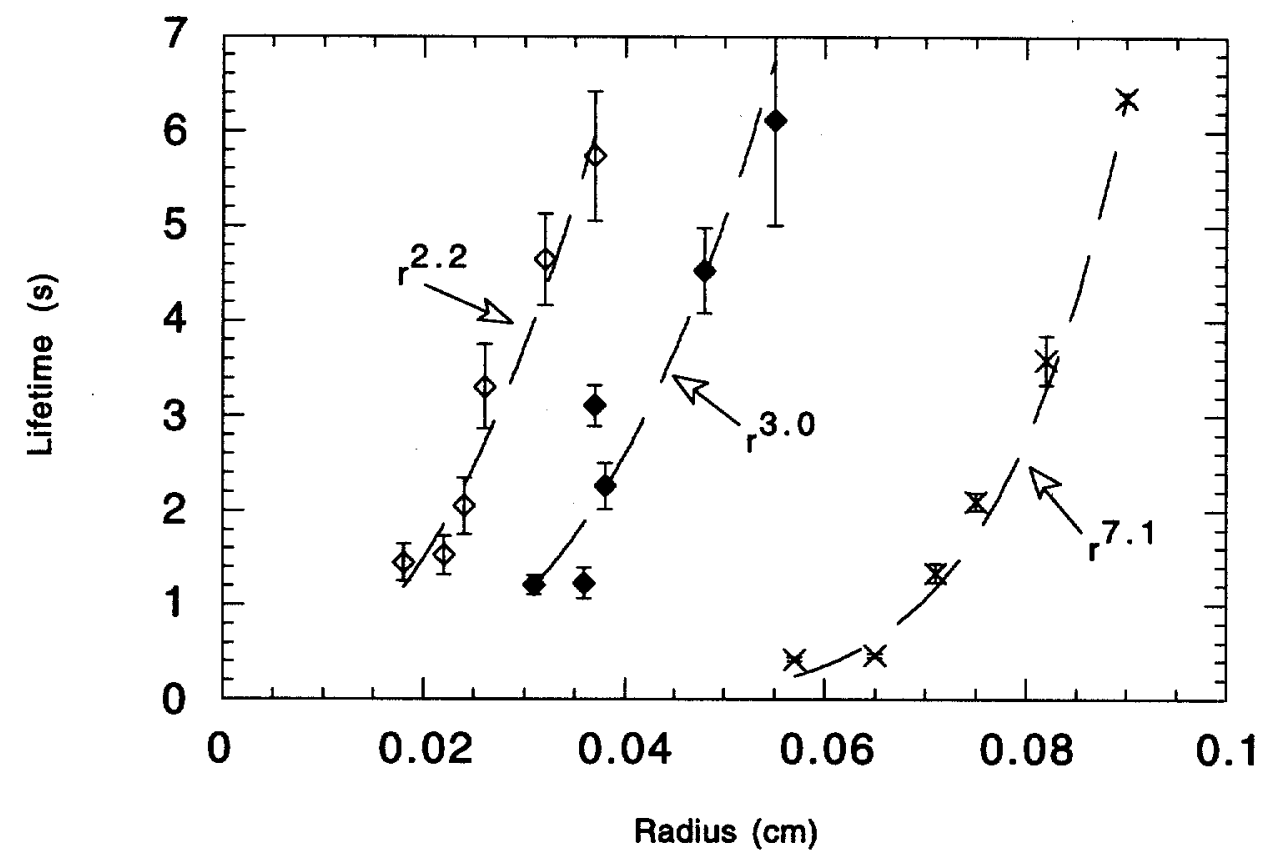

Figure 4.20 Radial dependence, $\mathrm{L}_{0}=18 \mathrm{~cm}, \mathrm{~B}=30 \mathrm{kG}, \mathrm{n}=1.7 \times 10^{8}(0), 7.0 \times 10^{7}(\bullet)$ and $2.2 \times 10^{7}(x) \mathrm{cm}^{-3}$. Curves are fits to a power law: $\alpha=2.2 \pm 0.3,3.0 \pm 0.8$, and $7.1 \pm 0.5$, respectively.

improve. Given the large error bars in the lifetime fit at short times, it is difficult to draw solid conclusions.

The extremely high-density evolution seen in Fig. 4.19 is one of the few cases in this study where the captured electrons form a true three-dimensional plasma. It is also the case that falls closest to the V' lifetime curve, both qualitatively and quantitatively. The plasma is at a much higher density than the $V^{\prime}$ plasmas, but because of the smaller $r_{p}$, the two systems have equivalent values of $\lambda_{D} / r_{p} \leq 0.1$. 


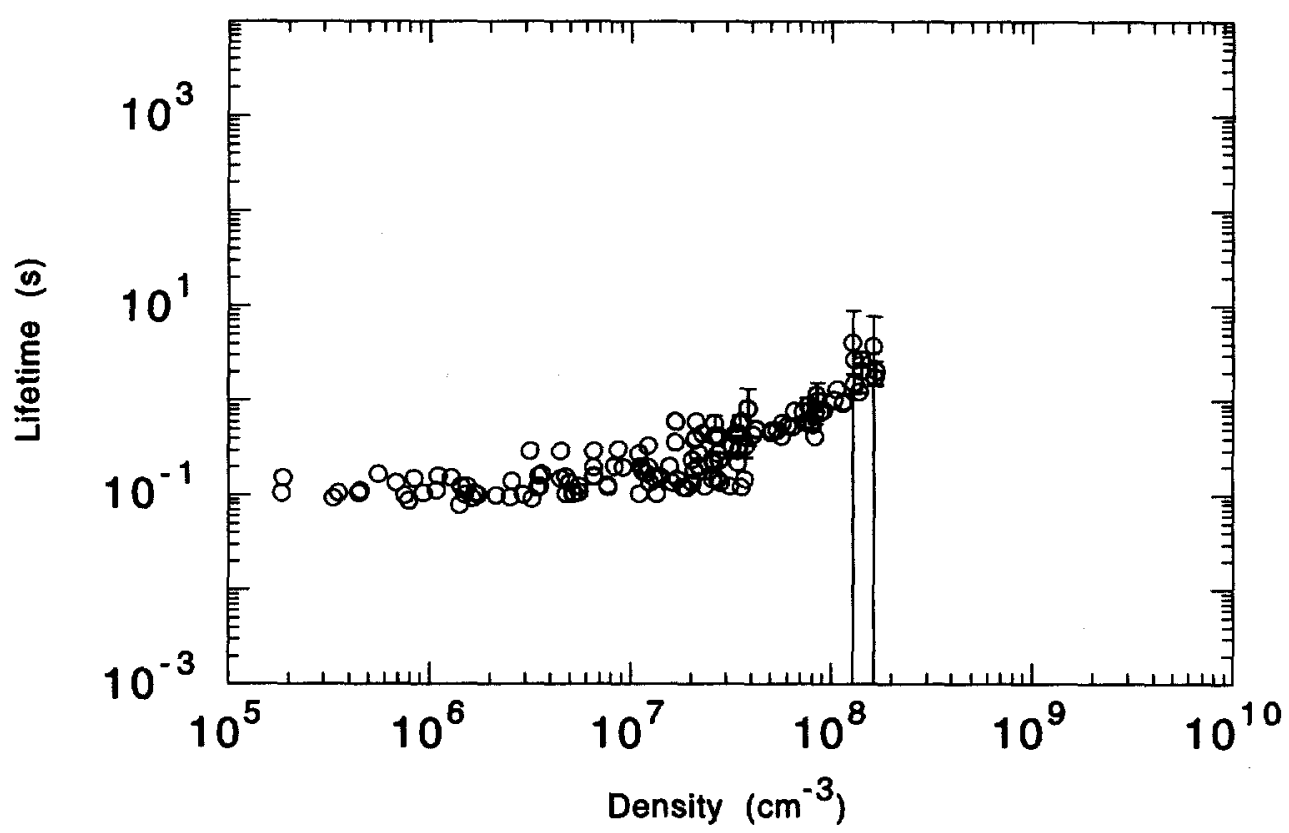

Figure 4.21 Slowly decreasing lifetime during evolution, $L_{0} \geq 12 \mathrm{~cm}, \mathrm{~B}=30 \mathrm{kG}$, $\mathrm{n}_{0}<3 \times 10^{8} \mathrm{~cm}^{-3}$.

More difficult to understand is the low-density evolution. Fig. 4.21 plots all the data sets for $L_{0} \geq 12, B=30 \mathrm{kG}$, and $n<3 \times 10^{8} \mathrm{~cm}^{-3}$. The slow proportionality between density and lifetime appears largely independent of both $L$ and $r_{p}$. This is consistent with the length independence seen for long electrodes in the half-life analysis (Fig. 4.9). Fig. 4.22 shows that, as $B$ is decreased, the early evolution changes dramatically. The lifetime initially increases rapidly before stabilizing. This change occurs in the region where cyclotron cooling ceases to be significant on the confinement timescale, and so may be 


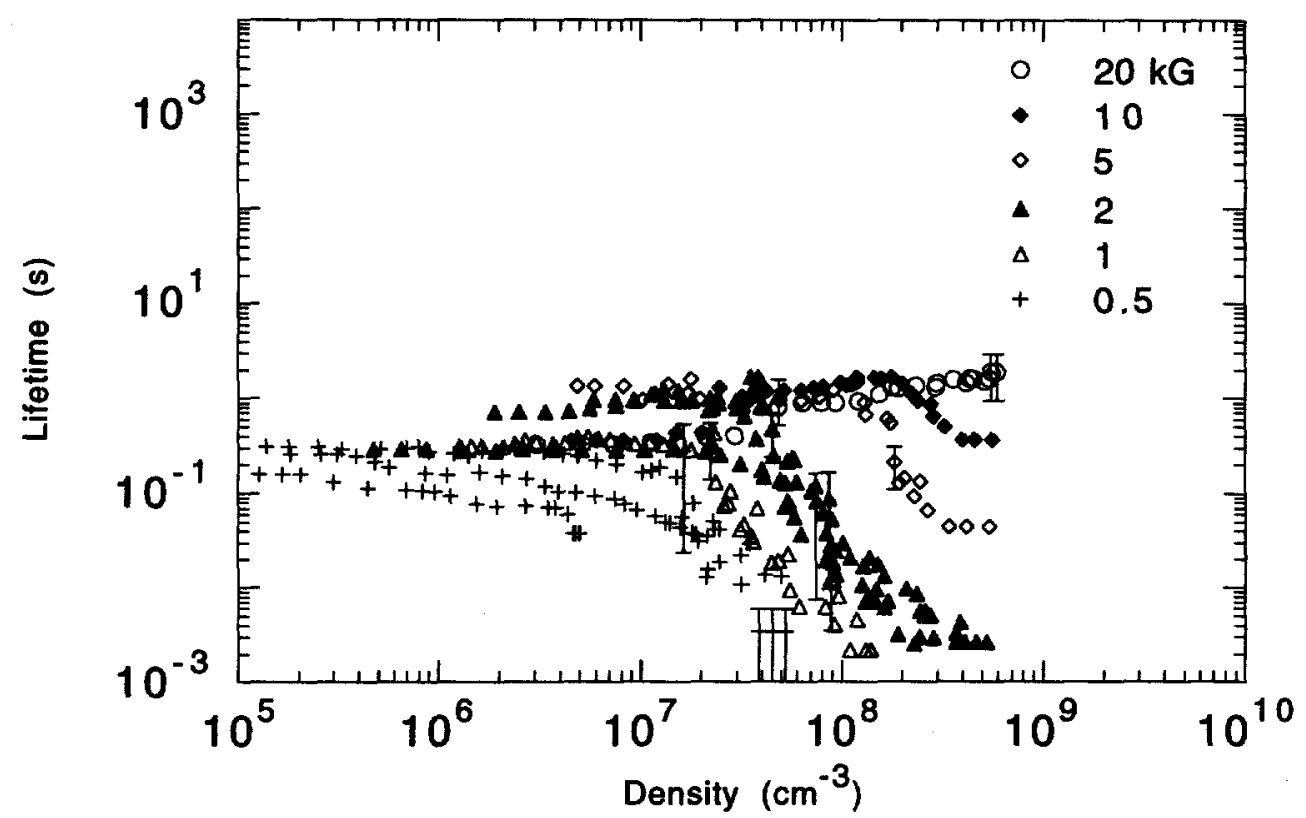

Figure 4.22 Transition from high-field to low-field evolution, $\mathrm{L}_{0}=18 \mathrm{~cm}$. All series evolve to a very similar final $\tau_{i}$.

a temperature-related phenomenon. It also falls in the regime where the cyclotron orbit is becoming significant and initially $\rho / r_{p} \rightarrow 1$. The cyclotron radius itself is temperature dependent, so it is difficult to decouple the various effects in this region.

The most interesting feature in both Figs. 4.21 and 4.22 is the fact that all the plasmas evolve toward a lifetime $1.0<\tau_{i}<0.1$. This final state shows no systematic dependence on $T, r_{p}$, or $B$. Longer electrodes at low $B$ evolve to the same range, so there is once again 
no dependence on $L$ in the long plasmas. The important factor in defining this regime may be the electron-electron collision time,

$$
v_{e} \times 3 \ln \Lambda \mathrm{s}^{-1}\left(\frac{n}{10^{6} \mathrm{~cm}^{-3}}\right)\left(\frac{T}{1 \mathrm{eV}}\right)^{-3 / 2} .
$$

The Coulomb logarithm $\ln \Lambda \approx 15-20$. When $n \approx 10^{6} \mathrm{~cm}^{-3}, v_{e} \tau_{i} \approx 1$, at which

point the system is effectively collisionless. The approach to $\tau_{i}=0.1$

then represents the move toward single-particle confinement.

The remaining mystery comes from the robust value of $\tau_{i}$ in this limit. Single-particle transport across magnetic field lines is nominally a simple matter, and routinely calculated for given electric and magnetic field errors; however, all of these mechanisms are strongly dependent on the magnetic field strength, and often on the temperature as well (see Appendix A).

A clue to the process may be found in low-field evolution in short electrodes, shown in Figs. 4.23 and 4.24. The rapid increase in $\tau_{i}$ is similar to the early evolution in longer electrodes; but the 


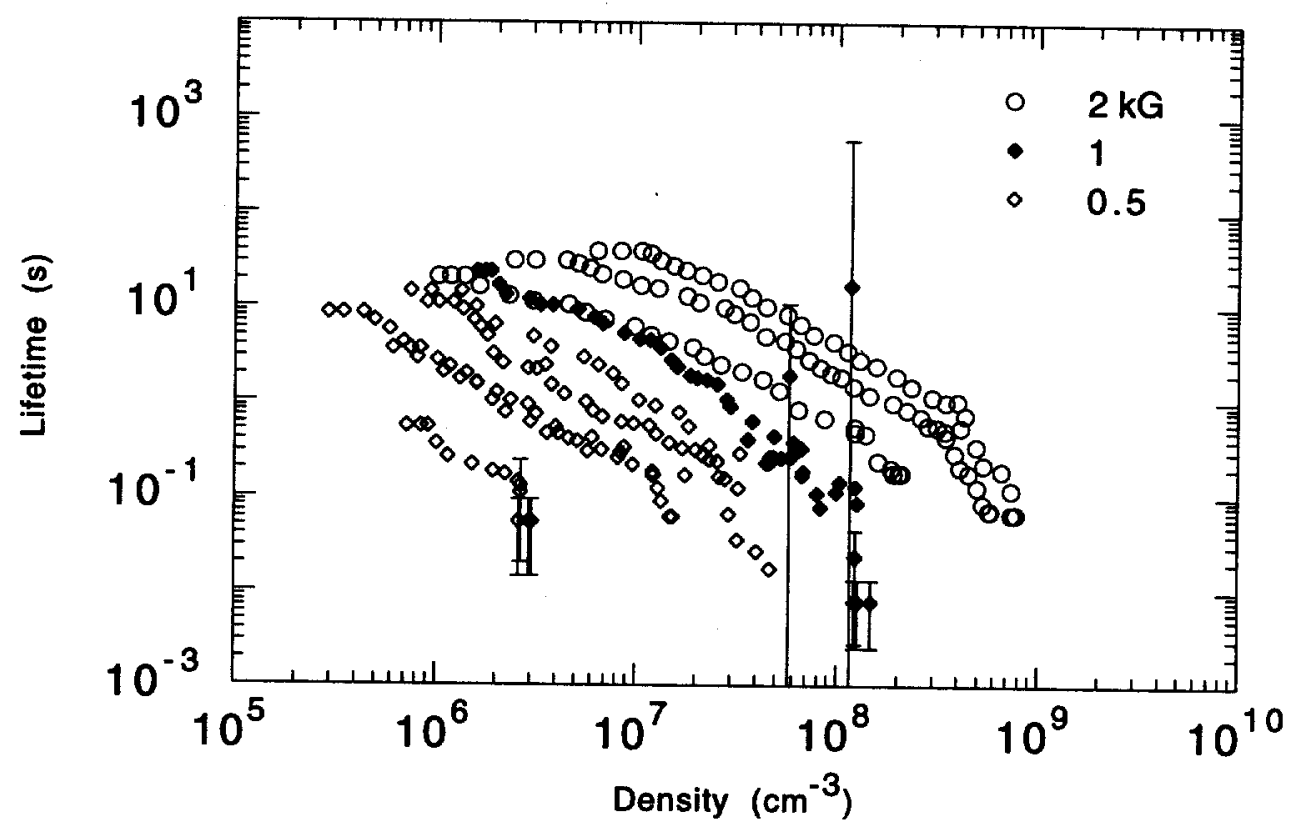

Figure 4.23 Low-field plasma evolution in $6 \mathrm{~cm}$ electrode.

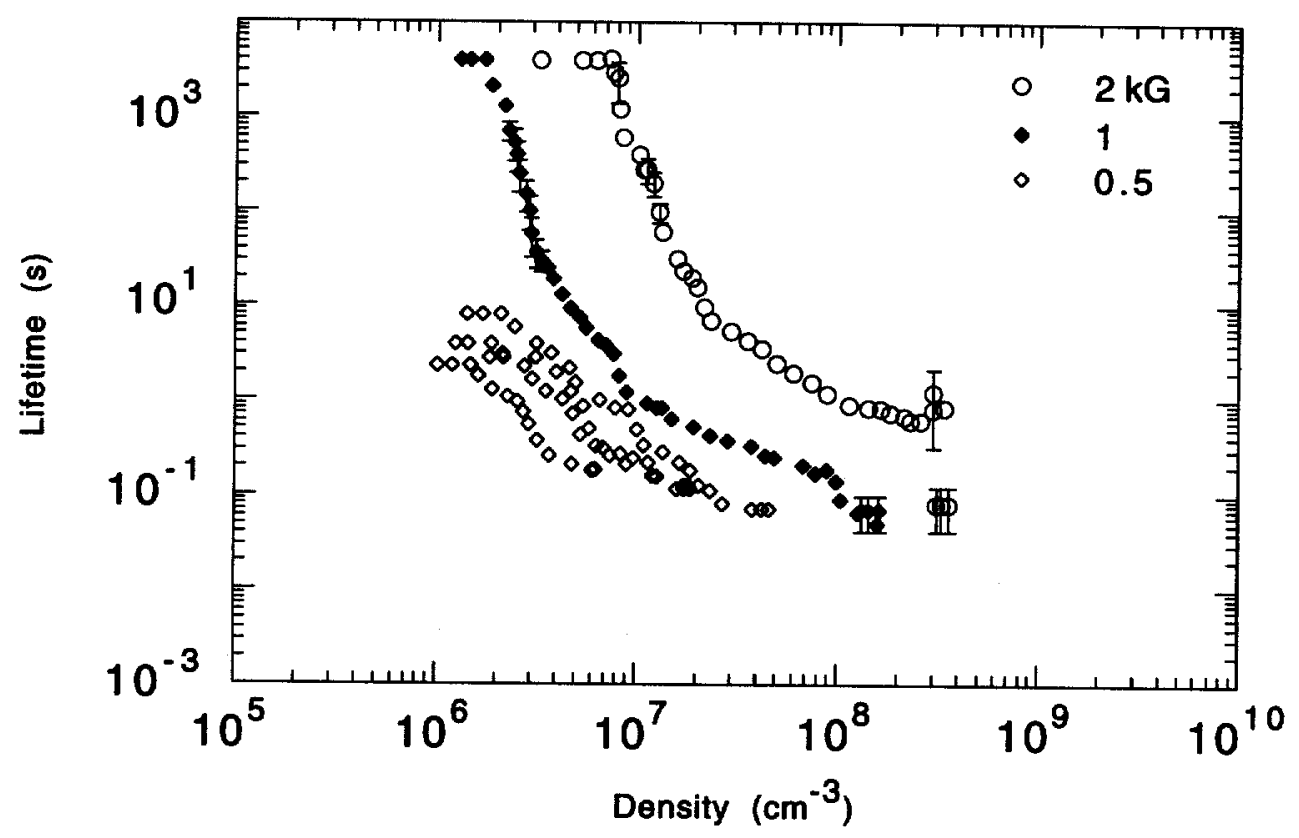

Figure 4.24 Low-field evolution in $2 \mathrm{~cm}$ electrode. 
"saturation" mechanism that halts the improvement is apparently absent. As discussed in reference to the diocotron measurements, the electrodes produce significant radial electric fields throughout the short trap, while longer traps have a significant field-free drift region. The potentials in the shortest traps approximate those in the traditional hyperbolic-electrode Penning traps used to confine individual particles for long periods.

The radial fields can stabilize drifts associated with field errors (see Appendix C). The difficulty with this explanation is that even the low bounce-averaged radial electric fields in long electrodes are large enough, in the single-particle calculation, to stabilize significant DC errors, e.g. $10 \mathrm{mV} / \mathrm{cm}$ stray electric fields and magnetic field curvature of radius $10 \mathrm{~m}$. Perhaps the losses are caused by noise or other time-dependent variations.

In summary, the electron confinement appears to be characterized by three regimes. The high-density "true plasma" regime exhibits confinement characteristics similar to that seen in previous nonneutral plasma devices. As $\lambda_{D} \rightarrow r_{p}$, the electrons enter a narrow, 
transitional "quasi-plasma" regime, in which the confinement ceases to improve as density is decreased. As the density approaches the collision-free limit, confinement degrades from the transitional peak, and approaches a fundamental limit $\approx 0.5 \mathrm{~s}$ that is insensitive to most trap parameters. The one way to overcome this limit appears to be to confine particles in a small electrode with a Penning potential structure. 


\section{Discussion}

\subsection{Conclusions}

The confinement limitations of Penning-Malmberg traps, demonstrated with electrons, have clear implications for future positron work on this device. Successful TOF positron accumulation will require substantial manipulation to transfer positrons from a long capture section to short storage regions. In addition, the manipulation must be done without heating the positrons above the positronium formation threshold. The techniques discussed in Chapter 3 are by no means exhaustive, but should serve as a starting point. Once a density near $10^{8} \mathrm{e}^{+} / \mathrm{cm}^{3}$ is accumulated, the length requirement may be relaxed, allowing a second stage of more efficient accumulation by collisional cooling.

The electron confinement studies answered the questions regarding the positron losses, but left many more questions about the fundamental limitations of Penning-Malmberg traps. The bounce- 
resonance model does not effectively characterize the behavior of this system in any of its details. Since the model does not require any collective interaction beyond collisions, it is not clear why plasma shielding is required to see its effects. Additional theoretical work is necessary to understand these results and develop a stronger connection between the deconfinement candidates discussed in Appendix A and their experimental manifestations.

\subsection{Positron Improvements}

Positron accumulation could be improved by modifications to the vacuum system, the trap control system, the positron beam, and the remoderator foil. The vacuum in the trap is difficult to characterize, because of the failure of ion gauges in the high magnetic field. Nonetheless, it is clear that the vacuum is significantly degraded, perhaps by an order of magnitude, when the trap is open to the positron beamline. The situation is made worse by the fact that the cold copper bore is the dominant pumping region. Cooled baffles mounted in the stainless-steel transition region between the copper bore and the room-temperature region would significantly reduce 
positronium formation. Such baffles were designed for operation of the trap at $\mathrm{LHe}$ temperature to minimize radiative heating, but could also serve as a differential pumping stage at $\mathrm{LN}_{2}$ temperatures.

Reducing positronium losses would allow the use of larger trap potentials and faster switching.

The trap manipulations required to maneuver the positrons after capture may require an improved control system. The present system is only able to switch potentials between two levels, with variable slew rates. This was a significant limitation for the crenelated potential schemes, because the periodic wells could not be completely eliminated when dumping cold positrons from the trap. In addition, the optimal trap configuration may change as the density of accumulated positrons increases. More refined, multi-level control would solve both of these problems.

The electron temperature measurements clearly demonstrate the need for noise filters on the power supplies for any low-temperature experiments. Relays will be required to short out the filters during voltage switching. This is particularly important for Bhabha 
scattering, because of the significant electron beam heating and the necessary precision in the collision energy.

The positron beam from the linac is the brightest source available, but in principle it could also be improved. No systematic study of the positron production mechanism has been performed. For its original purpose as a materials science probe, the existing beam is already too intense and must be attenuated. Simple modifications made during these trapping experiments have doubled the production efficiency, and additional increases of similar magnitude are almost certainly possible.

The most significant increases in initial positron capture efficiency may come from the remoderator. The foil could be annealed in situ by an electron beam or insertable filament. Annealing increases the positron diffusion length in the foil by removing crystal defects and evaporates surface contaminants that can interfere with positron emission. Moderation efficiency can increase by as much as a factor of 4 . 
Moving the remoderator to the downstream end of the trap may also be useful. Reflection moderation is more efficient than the transmission configuration now in use. Placing the moderator between the trap and the imaging diagnostics presents its own logistical problems, but given the large benefits associated with quickly accumulating a large density, the possibility deserves further study.

The most promising prospect for improving confinement is the "rotating-wall" technique developed for ion trapping [46]. A multiply-segmented electrode can be used to drive an azimuthal wave in the plasma. If the frequency of the rotating wave is slightly faster than the natural $E \times B$ rotation of the charge, the wave will accelerate the rotation and compress the plasma. This has been used to increase the density of an ion plasma to $10 \%$ of the Brillouin density, the theoretical maximum density achievable in a given magnetic field.

Among the more esoteric possibilities for increasing accumulation is the use of chaotic scattering $[47,48]$. Non-adiabatic electric field 
gradients have been found to produce dramatic coupling between the transverse and longitudinal motion of reflected positrons, allowing significant accumulation even in a static trap configuration. The challenge in applying this technique is the generation of significant field gradients on the micron spatial scale of cyclotron motion. The phenomenon was studied in $B<100 \mathrm{G}$, with high-transmission grids at $V \approx 500 \mathrm{~V}$ as barriers.

In principle, the chaotic reflection point could be placed in the kiligauss fringe field at the downstream end of the magnet, for capture of a high-velocity beam. Static field accumulation may even occur in the fringe region as scattered particles are mirrored by the high magnetic field. Cyclotron cooling would eventually allow the slowed particles to re-enter the high field region. Since this system could obviate the need for a remoderation foil, an efficiency of only $10-20 \%$ would still represent a significant improvement over the existing system. 


\subsection{Electron Improvements}

As discussed in Sec. 2.3, the electron gun design was not optimized for low-voltage beams. The earlier stitched spiral filament was found to give very irregular injection due to uneven heating. Improving both of these systems to provide two independent electron sources would be useful for future work. For proper simulation of the positron beam, an improved small-diameter gun in the fringe field is still ideal.

For plasma experiments, a sturdy spiral filament should be placed in the high field at the front end of the trap. The spiral filament is more standard for non-neutral plasma experiments, and could be used to generate true plasmas, with Debye shielding in all dimensions. The larger number of particles would also make plasma waves easier to produce and detect, which is a critical diagnostic for studying wavedriven deconfinement mechanisms. In addition, the spiral could be "tapped" at multiple points to allow generation of plasmas with various radii. An insertion system that allowed independent movement of both the filament and the moderator would be ideal, 
allowing the filament to double as a foil annealing unit.

\subsection{Future Experiments}

The future for the Bhabha scattering experiment is unclear. Recent heavy-ion experiments have cast doubt on the earlier observations of anomalous positron-electron pair production [49]. There is still considerable controversy surrounding the subject, and additional heavy-ion experiments are necessary to decide the issues and justify the considerable engineering work required to produce the highdensity positron plasma target. In order to perform the Bhabha scattering experiment, the positrons must still occupy a long interaction length, while remaining well-confined. The complex manipulations required during accumulation will also reduce the overall efficiency of the experiment by increasing the trap "dead" time.

This unique apparatus can be useful in other positron and electron studies. The interaction of positrons with gases in a high magnetic field is of interest in astrophysics [50]. The unusual square-root 
relationship observed between the positron lifetime and the gas pressure (Sec. 3.3) suggests that interesting physics is occurring. The recombination rate of gases in a high field is also a subject of theoretical interest [51]. At low energy, three-body interactions dominate, but in high fields the closest approach may be substantially larger than a cyclotron radius. Trapped particles then interact two-dimensionally as rods of charge. A beam of low-energy electrons propagating through a positron cloud would provide an excellent probe of this process. The gamma radiation resulting from positronium formation provides a straightforward measurement of the recombination rate.

The availability of both positrons and electrons could allow studies of positron-electron plasmas. Such plasmas are of great theoretical interest [52,53,54]. Much of classical plasma physics assumes that the electron mass is negligible relative to the ion mass. When the plasma components are of equal mass, many classical phenomena disappear, such as Faraday rotation of light polarization and the quasilinear three-wave decay mode. Equal-mass ion plasmas have been formed, and show evidence of strong turbulence, in which the wave energy 
density is much higher than the particle kinetic energy [55].There are also analogues to systems such as electron-hole plasma modeling of semiconductors, such as germanium [56]. The confinement of neutral plasmas of course poses its own substantial challenges. Experiments with positrons in this area have thus far focused on beam-plasma interactions using trapped positrons and a low-energy electron beam [57].

Clearly, the deconfinement mechanisms in Penning-Malmberg traps are still poorly understood. The difficulties faced in the positron work demonstrate the dangers associated with extrapolation from empirical "laws." The large parameter space accessible in this apparatus makes it a powerful system for the testing of future confinement theories. 


\section{Appendix A Particle Deconfinement}

\section{A.1 Transport Theories}

As discussed in Sec. 1.3, the basis of the confinement theory for nonneutral plasmas is the conservation of angular momentum. Transport leading to deconfinement results when angular momentum is added to the plasma. Angular momentum can be added to the plasma through single particle interactions as well as collective plasma response. A simple theory of deconfinement due to collisions with neutral particles suggests a lifetime that scales as $\tau \propto B^{2} / p$, where $p$ is the background gas pressure [58]. This process is a single particle phenomenon, and not dependent on any collective plasma behavior.

Static field errors can drive basic single-particle drifts. Electric field errors with an azimuthal component drive $E \times B$ drifts in the radial direction. Curvature of the magnetic field causes $\nabla \boldsymbol{B}$ drifts [59], 


$$
\mathbf{v}_{\nabla}=\frac{\left(\mathbf{v}_{1}^{2}+\frac{1}{2} \mathbf{v}_{0}^{2}\right)}{R_{B} \Omega}\left(\hat{R}_{B} \times \hat{B}_{0}\right)
$$

where $R_{B}$ is the radius of curvature.

Field errors can also induce single-particle transport through bounceresonance [22]. Particles with a bounce frequency $\omega_{b}$ and rotation frequency $\omega_{r}$ satisfying the resonance condition $k \omega_{b}+l \omega_{r}=0$, where $k$ is an integer and the field has a component of the form $e^{i l \theta}$, will gain angular momentum and move outward in radius. Interactions between particles are required only to repopulate the resonant region of phase space. Collective processes are ignored in this theory; the dominant effect is the transfer of angular momentum to incoherent particle motion. This theory suggests that confinement lifetime should scale as $\tau=f\left(\omega_{b} / \omega_{r}\right)$. The exact nature of the function $f()$ is not known a priori from the theory.

It has been shown that static field errors can also enhance transport through collective processes $[60,61]$. Because the plasma is rotating, 
it can support a mode that has zero frequency in the laboratory frame. This mode can be resonantly driven by static field asymmetries. While a zero frequency mode cannot add energy to the plasma, it does carry angular momentum, which can be transferred to the plasma as a whole if the mode is Landau damped. In this theory, the transport due to a magnetic field error, $\Delta P_{\theta}(t)$, should scale as $\left.\left|\delta B_{r}\right| B_{0}\right|^{4 / 3}$, and should increase linearly with time until the mode saturates.

The limitation of the linear theory is that a zero frequency mode is not guaranteed for arbitrary plasma parameters. Nonlinear treatment of the plasma allows enhanced transport through threewave interactions and beat-resonant coupling $[62,63,64]$. In the three-wave instability, the static field can drive two daughter waves, subject to the resonance condition,

$$
\omega_{p 1}+\omega_{p 2} \approx 0
$$

The daughter waves have zero net energy but non-zero angular momentum. Negative energy modes, like zero energy modes, are allowed because the plasma is rotating. The addition of a third mode 
in the nonlinear theory allows the process to occur in a broader range of plasma parameters than the linear theory.

Beat-resonant coupling, or induced scattering, is similar to the threewave instability except that one mode is replaced by resonant particles. Particles resonate with the beat frequency of the static perturbation and a plasma mode. In this case, the resonance requirement is extremely loose. The only constraint is that a mode frequency exists in the plasma such that some of the plasma particles are capable of Landau damping the resulting beat wave. The weak resonance constraint suggests that beat-resonant coupling is the most generally applicable process; however, estimates of the instability thresholds for laboratory conditions suggest that beat-resonant coupling requires field errors as much as an order of magnitude larger than the three-wave decay instability [64].

Recent transport studies have attempted to apply neoclassical transport theory to these nonneutral systems $[65,66,67]$. Neoclassical transport applies in situations where $\rho \ll \lambda_{D}$, which is generally the case in these devices. Classical Boltzmann theory assumes that 
particle collisions are predominantly binary scattering with an impact paramter $b$ in the range $b_{\min }<b<\rho$, where $b_{\text {min }}=e^{2} / k T$ is the distance of closest approach. The step size for diffusion is $\rho$, leading to a diffusion coefficient $D_{\text {class }} \approx v_{e} \rho^{2}$, with the electron-electron collision rate $v_{e}$ as discussed in Sec. 4.3,

$$
v_{e} \approx 3 \ln \Lambda \mathrm{s}^{-1}\left(\frac{n}{10^{6} \mathrm{~cm}^{-3}}\right)\left(\frac{T}{1 \mathrm{eV}}\right)^{-3 / 2}
$$

and the Coulomb logarithm for a magnetized plasma $\ln \Lambda=\ln \left(\rho / b_{\text {min }}\right)$.

In a highly-magnetized plasma, the dominant interaction is instead a long-range $\boldsymbol{E} \times \boldsymbol{B}$ drift collision [65], with impact parameter in the range $\rho<b<\lambda_{D}$. Trap particles with similar velocities can interact for long times. Calculation of the diffusion coefficient requires integrals over the paths of interacting particles, but is of order $D_{E \times B} \approx D_{\text {class }} \ln \left(\lambda_{D} . / \rho\right)$.

A first-order neoclassical calculation, integrating along unperturbed 
orbits, has been compared to test-particle transport in a nonneutral ion plasma [67]. The measured transport is more than an order of magnitude greater than the classical prediction, and only a factor of three above the neoclassical result. There are considerable uncertainties in the neoclassical calculation depending on assumptions about velocity scattering rates and the orbit integrals.

Because these collisions involve identical particles, they should conserve angular momentum, enhancing transport to thermal equilibrium, but not producing any bulk deconfinement. The increased diffusivity does impact the interaction of trapped particles with both static field errors and collective waves, so further theoretical work in this region may shed light on the deconfinement mystery.

\section{A.2 Electron Experiments}

Most of the processes discussed in the previous section can be induced and have been observed experimentally, but it is still 
unclear which is the dominant deconfinement mechanism when none are intentionally driven. Studies of how electron plasma confinement scales with various experimental parameters have been performed at the University of California, San Diego and Berkeley.

In the San Diego devices, the electron plasma evolution follows the neutral-particle collision theory quite well at pressures above $10^{-6}$ Torr [68], but the pressure scaling breaks down at lower pressures [15], where the lifetime stops improving. The anomalous loss does not appear to be an effect of a gas species with large collision cross-section. Instead, the loss is attributed to one of the field asymmetry mechanisms.

Confinement experiments with no applied perturbation find a pressure-independent lifetime scaling of $\tau \propto(L / B)^{-2}$ in the low pressure regime [24]. Although the qualitative nature of the transport varies in different parameter regimes, the basic scaling holds roughly for lifetimes ranging from $3 \times 10^{-3}$ to $10^{3}$ seconds. As shown in Fig. A.1, the scatter in the data is nearly an order of 


\section{This space reserved for UCSD confinement plot.}

Figure a.1 UCSD confinement vs. L/B and bounce-resonance ratio. Reproduced from Ref. 69 with permission of authors.

magnitude, due primarily to reproducible variability of the lifetime in different electrodes of the same length.

The $L^{-2}$ length dependence can be obtained from the single particle resonant transport theory discussed above. This treatment requires ad hoc assumptions about the nature of static field errors, which change for different ranges of $B / L$. The magnetic field dependence 
also arises from resonant transport, but $B^{2}$ scaling is a characteristic of cross-field diffusion in general. Assuming the transport is due to bounce resonance, the lifetime would scale as $\tau \propto\left(\omega_{b} / \omega_{r}\right)^{2}=(B / n L)^{2} T$ [36]. Additional experiments with high density plasmas in a cryogenic device find fair agreement with the bounce-resonant extrapolation from EV and $V^{\prime}$ data, but no fundamental understanding of the mechanism has been achieved $[45,69]$.

The linear mode resonance has been observed by placing a nonaxisymmetric perturbation with variable frequency on confinement electrodes [70]. It is easier to find the resonance using a variable frequency perturbation than applying a static perturbation and varying the plasma parameters. Transport is observed to increase when normal modes of the plasma are resonantly excited.

Nonlinear enhancement of transport has also been observed, and is attributed to beat-resonant coupling [70,71]. An applied perturbation results in the growth of a single mode at a different frequency. The instability occurs over a broad range of parameters, and enhances 
the radial transport by over an order of magnitude. This last point is significant because the excited mode is azimuthally symmetric and cannot by itself cause radial particle transport. Thresholds and growth rates are also consistent with the two-wave beat-resonant theory. It is notable that this instability can be easily stabilized by placing a resistance between a confining electrode and ground. This enhances mode damping beyond that from wave-particle effects and substantially reduces the radial transport.

Systematic studies of the confinement behavior have also been performed at Berkeley [72]. Confinement in the Berkeley apparatus scales as $L^{-1.4 \pm 0.15}$ and $B^{2.19 \pm 0.03}$, when no field perturbations are applied. This is roughly consistent with the San Diego scaling, with similar irregularities in the length data. Of particular note is the dependence on background gas pressure. Over four decades in range, the confinement time scales as $p^{-0.5}$. This is unusual because it suggests that the confinement is not linearly dependent on the collision frequency. No explanation has been proposed.

When strong asymmetric electrostatic perturbations are applied in 
the Berkeley device, the confinement scaling with magnetic field changes. The exponent of the magnetic field dependence is $0.45 \pm 0.06$ for positive perturbations and $0.80 \pm 0.04$ with negative applied voltages. This is far from the expected value of 2 for diffusive transport, and suggests that a collective process may be involved.

With an applied perturbation, no temperature dependence in the lifetime is observed, in contrast to the San Diego findings. The density dependence is coupled somewhat to the size and polarity of the applied perturbation, but roughly follows $\tau \propto n^{-1.3 \pm 0.15}$. This does not appear to be in agreement with the prediction of bounceresonance theory.

Overall, the confinement behavior in these devices, while fairly systematic on individual machines, is not solidly reproducible between devices. It is still unclear which of the proposed deconfinement mechanisms, if any, dominates the transport in each device. Without this understanding, it is difficult, if not impossible, to predict with any certainty what the confinement of a newlyconstructed device will be. 


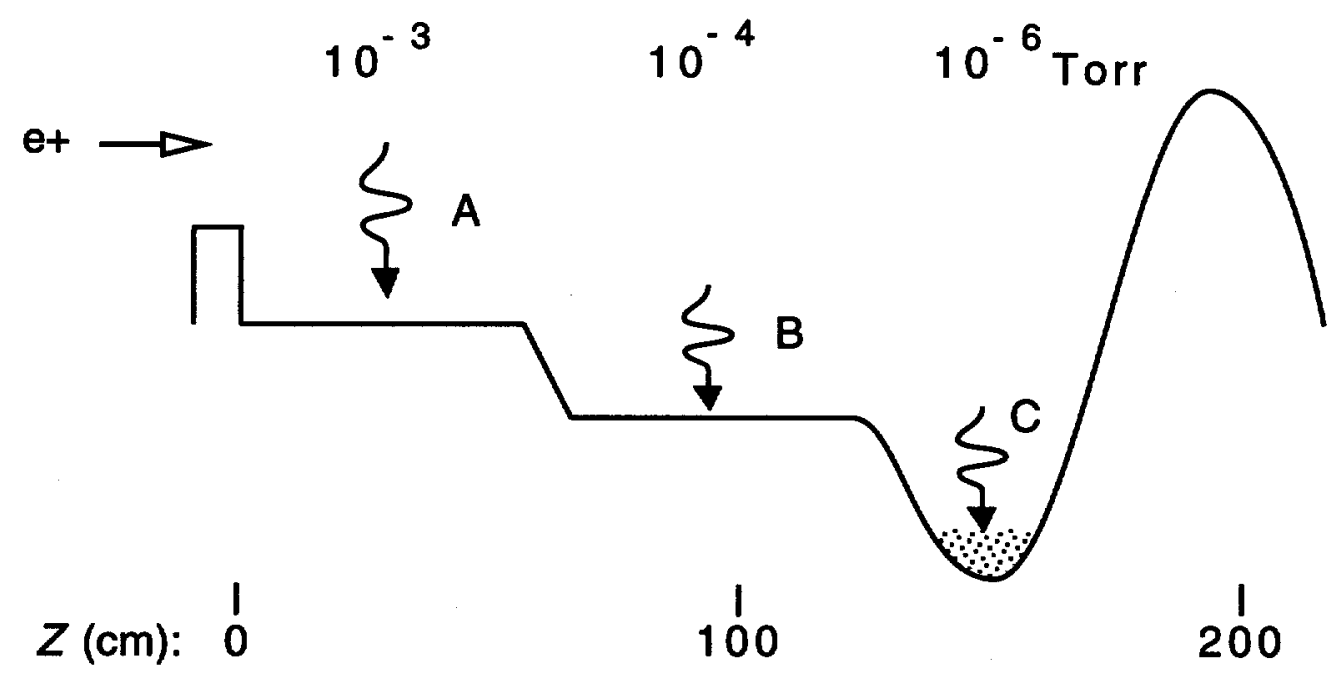

Figure a.2 UCSD three-stage positron trap potential. Positrons scatter from electronic level of $\mathrm{N}_{2}$ in one pass (A). They scatter from a vibrational level after $\approx 1 \mathrm{~ms}(\mathrm{~B})$, and again after $\approx 20 \mathrm{~ms}$ (C).

\section{A.3 Positron Experiments}

At UCSD, positrons are accumulated in a Penning-Malmberg trap from a radioactive source [73]. The mechanism for accumulation is scattering from $\mathrm{N}_{2}$. Fig. A.2 shows a schematic of the trap apparatus.

Positrons must make an inelastic collision in one pass through the first trap region in order to be captured. Subsequent collisions cool the positrons into deeper potential regions of the trap. Note that in the final confinement region, $L / D \approx 1$, so radial electric fields are large. Positron confinement in this configuration is substantially shorter 
than would be expected from electron studies. The anomalous deconfinement is caused by enhanced annihilation with large organic molecules, in particular vacuum pump oil [74]. The complex structure of the molecules results in spatial regions where positrons can enter a quasi-bound state, resulting in a large increase in the annihilation cross section, and a rapid loss of positrons from the trap. The use of cryopanels in the trap to eliminate the pump oil improves confinement by several orders of magnitude [75]. Table A.1 summarizes and compares the three basic stages of the positron trapping experiment.

\begin{tabular}{l|ccc}
\hline & UCSD I & UCSD II & UCSD III \\
\hline Source (mCi) & ${ }^{22 \mathrm{Na}(1)}$ & ${ }^{22 \mathrm{Na}(88)}$ & ${ }^{22 \mathrm{Na}(88)}$ \\
\hline $\mathrm{N}_{\mathrm{e}+}$ & $?$ & $3.3 \times 10^{5}$ & $1.6 \times 10^{7}$ \\
\hline $\begin{array}{l}\text { Pressure } \\
\text { (Torr) }\end{array}$ & $5 \times 10^{-6}$ & $5 \times 10^{-6}$ & $\begin{array}{c}6 \times 10^{-7} / \\
7 \times 10^{-10}\end{array}$ \\
\hline $\mathrm{L}(\mathrm{cm})$ & 2 & 2 & 5 \\
\hline $\mathrm{r}_{\mathrm{p}}(\mathrm{cm})$ & 2 & 2 & 2 \\
\hline B (G) & 300 & 460 & 1400 \\
\hline Pumps & Turbo & $\begin{array}{c}\text { Turbo }+ \\
\text { Cryo }\end{array}$ & $\begin{array}{c}\text { Turbo }+ \\
\text { Cryo }\end{array}$ \\
\hline Lifetime (s) & 0.7 & 12 & $45 / 1700$ \\
\hline
\end{tabular}

Table a.2 Summary of positron trapping experiments at U.C. San Diego. 
Attempts to capture positrons from an accelerator have also found short lifetimes associated with poor vacuum [76,77]; however, in those cases the confinement is consistent with known positronium formation cross sections. The difficulty arose because the positrons were confined with energies greater than the $\approx 10 \mathrm{eV}$ positronium formation threshold. (See Appendix B.) Table A.2 summarizes the three accelerator experiments - two at Ghent University, Gent, Belgium, and one at the Electrotechnical Laboratory, Tsukuba, Japan.

\begin{tabular}{c|ccc}
\hline & Ghent I & Ghent II & ETL \\
\hline $\mathrm{N}_{\mathrm{e}+}$ & $1.3 \times 10^{5}$ & $1.3 \times 10^{5}$ & $?$ \\
\hline $\begin{array}{c}\text { Nominal p } \\
\text { (Torr) }\end{array}$ & $8 \times 10^{-7}$ & $8 \times 10^{-9}$ & $>3.5 \times 10^{-8}$ \\
\hline $\mathrm{L}_{0}$ (m) & 6 & 4.8 & 4 \\
\hline $\mathrm{r}_{\mathrm{p}}(\mathrm{cm})$ & $<1.5$ & $<1.5$ & 1 \\
\hline B (G) & 100 & 100 & 100 \\
\hline $\mathrm{E}_{0}$ (Volts) & 30 & 25 & 10 \\
\hline $\begin{array}{c}\text { Measured } \\
\text { Lifetime (ms) }\end{array}$ & 0.25 & 20 & 6.3 \\
\hline $\begin{array}{c}\text { Calculated } \\
\text { Lifetime (ms) }\end{array}$ & 0.24 & 26 & 9.5 \\
\hline
\end{tabular}

Table a.3 Summary of positron trapping experiments using an accelerator source. Calculated lifetime assumes annihilation cross section $\sigma=5 \times 10^{-16} \mathrm{~cm}^{2}$. 


\section{Appendix B Positron Interactions with}

\section{Residual Gases}

When positron energy is above the threshold $E_{P_{s}}$ for Ps formation with residual gas electrons, the annihilation cross section increases dramatically to $\sigma>10^{-16} \mathrm{~cm}^{2}$. A cross section of $5 \times 10^{-16} \mathrm{~cm}^{2}$ results in an annihilation time of only $100 \mathrm{~ms}$ for $10 \mathrm{eV}$ positrons in a $10^{-9}$ Torr vacuum.

Positronium has a binding energy half that of hydrogen, so $E_{P s}$ is $6.8 \mathrm{eV}$ below the ionization energy $E_{i}$ for a given molecule. The energy range $E_{P s}<E<E_{i}$ is referred to as the Orë gap [78]. $E_{P s}$ is only $5.5 \mathrm{eV}$ for $\mathrm{H}_{2} \mathrm{O}$ [79], $7 \mathrm{eV}$ for $\mathrm{CO}$ and $\mathrm{CO}_{2}$ [80], and $9 \mathrm{eV}$ for $\mathrm{H}_{2}$ and $\mathrm{N}_{2}$ [81].

Positronium formation is in competition with electronic excitation and dissociation of the molecule, which can cool positrons below $E_{P s}$. 
Collisional excitation results in an average positron energy loss of about $9 \mathrm{eV}$ for $\mathrm{N}_{2}$. This process has a threshold $E_{e x}=8 \mathrm{eV}$, and is more efficient than positronium formation up to $11 \mathrm{eV}$. The energy range in which the cooling is the dominant process is known as the "trapping gap." As discussed in Appendix A, this is utilized at UCSD in the accumulation of positrons through scattering on a buffer gas [82]. The dissociation threshold for $\mathrm{N}_{2}$ is relatively high, $E_{d}=10 \mathrm{eV}$.

Measurements with an RGA indicate that in the present apparatus, the dominant background gas at room temperature is $\mathrm{H}_{2}$, followed by $\mathrm{H}_{2} \mathrm{O}$ and $\mathrm{CO}$. Cooling to $77 \mathrm{~K}$ greatly increases the relative significance of $\mathrm{H}_{2}$. Since the RGA is in the room-temperature portion of the chamber, it is assumed that $\mathrm{H}_{2}$ is even more dominant in the trap itself. $\mathrm{H}_{2} \mathrm{O}$ is a significant scattering center at energies as low as $1 \mathrm{eV}$ because of its small $E_{d}$, but the inelastic scattering probability appears to diminish near $E_{P s}$, leaving annihilation as the dominant process [79]. Hydrogen is even more inconvenient, exhibiting a 
reduction in inelastic scattering around $E_{P s}$, and a large $E_{e x}=11 \mathrm{eV}$. Positronium formation is a significant process at the energies used in the present apparatus, and dominates the competing processes around $10 \mathrm{eV}$. Impact ionization, another potential cooling mechanism, begins to dominate over annihilation near $40 \mathrm{eV}$, the energy at which scattering into the potential well appears significant in the accumulation experiments. 


\section{Appendix C Single-Particle Ray Tracing}

\section{C.1 Numerical Calculations}

The confinement studies presented in Chapter 4 demonstrate that increasing the charge in a trap initially improves the trap's confinement properties. One possible explanation is that plasma shielding protects the majority of the particles from external field errors. Another factor that becomes significant as the charge approaches plasma density is the space charge potential. As discussed in Chapter 1, the electric field from the plasma itself creates the $\boldsymbol{E} \times \boldsymbol{B}$ plasma rotation. The angular momentum associated with this motion plays an important role in the basic confinement theory.

The radial electric field seen by the trapped particles is also increased in shorter lengths. The fact that both shorter length and larger density lead to improved confinement suggests that the radial 
fields rather than plasma shielding may be the relevent factor.

The importance of radial fields can be seen in numerical ray-tracing of the single-particle trajectory with applied field errors. The raytracing code [83] uses an ideal solenoid magnetic field, and electric fields calculated numerically from the actual electrode configuration and voltages [84]. Only a single particle is in the trap at a time, so dynamic effects from space charge, image charges, and wall resistance are ignored. For all the cases shown here, the test particle is launched at $0.01 \mathrm{~cm}$ radius, $60^{\circ}$ pitch angle relative to the $z$ axis, and $5 \mathrm{eV}$ kinetic energy. A field of only $1 \mathrm{kG}$ is used, because the large cyclotron frequency at larger fields forces very small time steps and leads to long calculation times.

The position of the particle is recorded each time it reflects at one end of the trap. Fig. C.1 shows the $x$ and $y$ coordinates of an electron in an $18 \mathrm{~cm}$ trap as a function of time, with no applied field errors. The fast oscillation is the cyclotron motion, and the slower orbiting motion results from magnetron drift, due to the radial electric fields produced in the fringe fields of the confining electrodes. The 


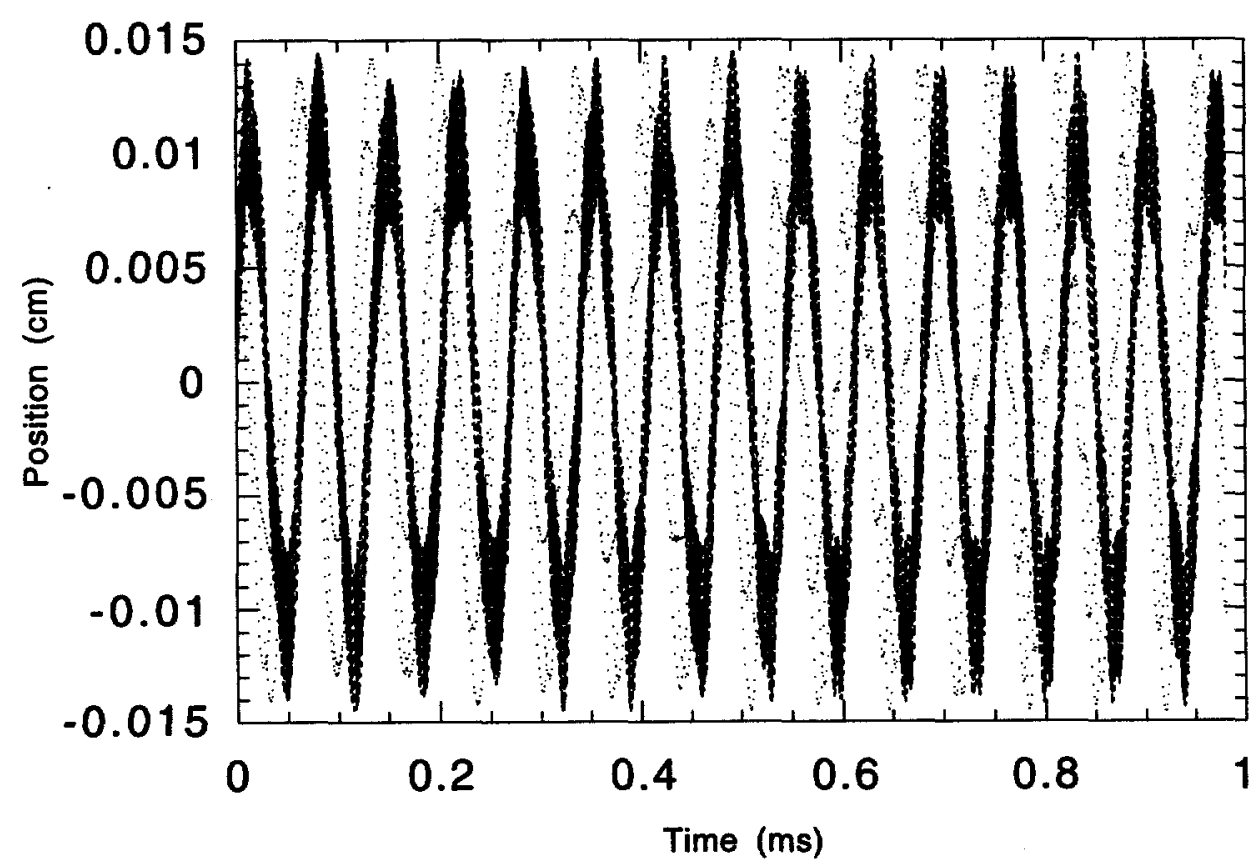

Figure c. 1 Numerical calculation of the orbit of a single electron, $L_{0}=18 \mathrm{~cm}$, $B=1 \mathrm{kG}$. The $\mathrm{x}$ (dotted) and $\mathrm{y}$ (solid) position is recorded after each round-trip in the trap.

magnetron frequency $v_{\mathrm{m}} \approx 1.43 \times 10^{4}$ indicates a bounce-averaged radial field,

$$
E_{r}=2 \pi v_{m} B r / c \propto 9 \mathrm{mV} / \mathrm{cm}
$$

Numerical averaging of the field seen by the particle during one bounce gives $10.1 \mathrm{mV} / \mathrm{cm}$. Note that this corresponds to the field that would be produced by a uniform plasma density of $1.4 \times 10^{7} \mathrm{~cm}^{-3}$ 


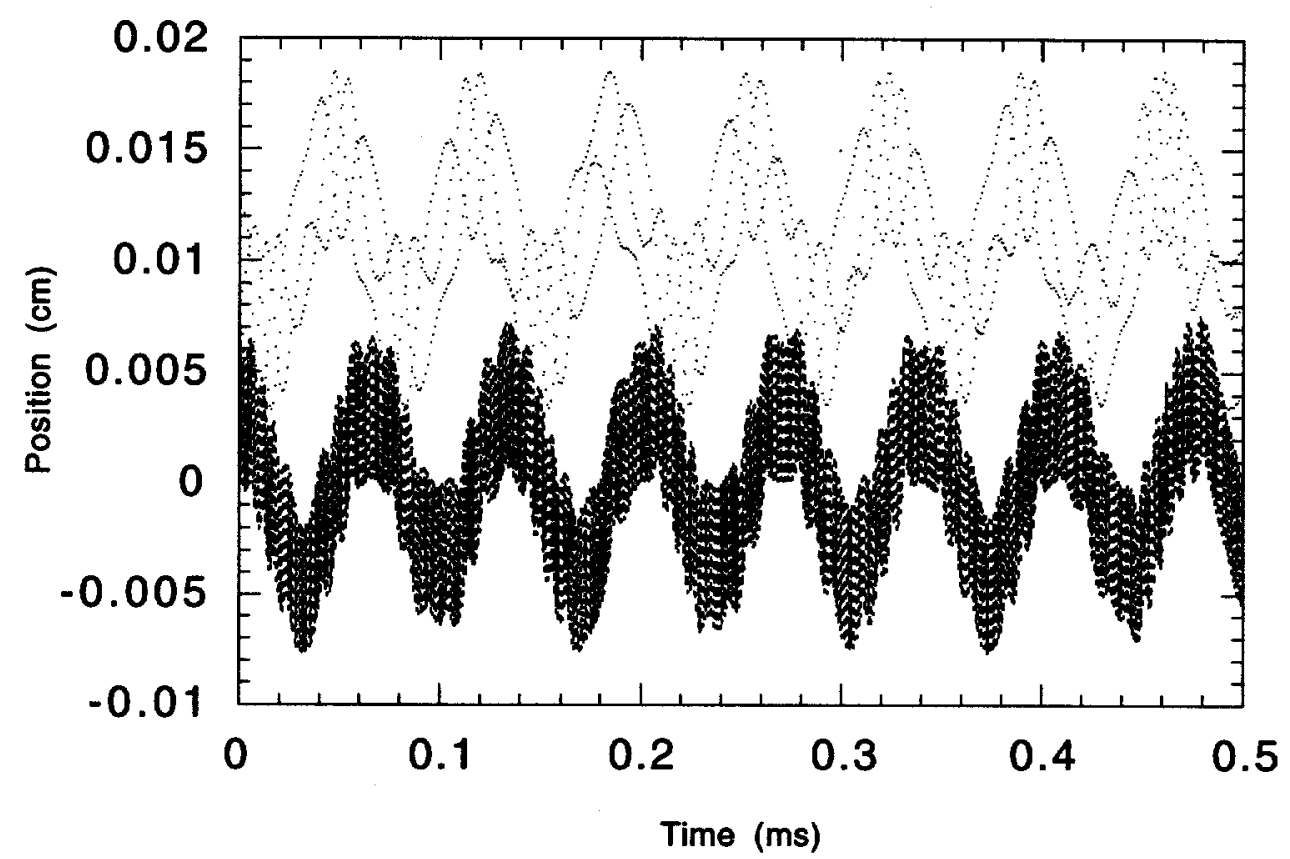

Figure c.2 Electron orbit in trap, same conditions as Fig. c.1, with a static electric field $E_{x}=10 \mathrm{mV} / \mathrm{cm}$. The orbit is stable but offset in $x$ (dots). The $y$ position (solid) is unaffected, although $\mathbf{E} \times \mathbf{B}$ is in the $\mathbf{y}$ direction.

\section{C.2 Electric Field Errors}

The ray-tracing is performed in three dimensions, allowing arbitrary static errors to be applied in any component of the electric and magnetic fields. Fig. C.2 shows the motion of an electron with an applied field $E_{x}=10 \mathrm{mV} / \mathrm{cm}$. The magnetron motion is offset from the center of the trap. The new orbit center occurs where $E_{r(\text { trap })}=E_{x(\text { error })}$. The $\boldsymbol{E} \times \boldsymbol{B}$ motion follows equipotential lines, and the applied "error" 


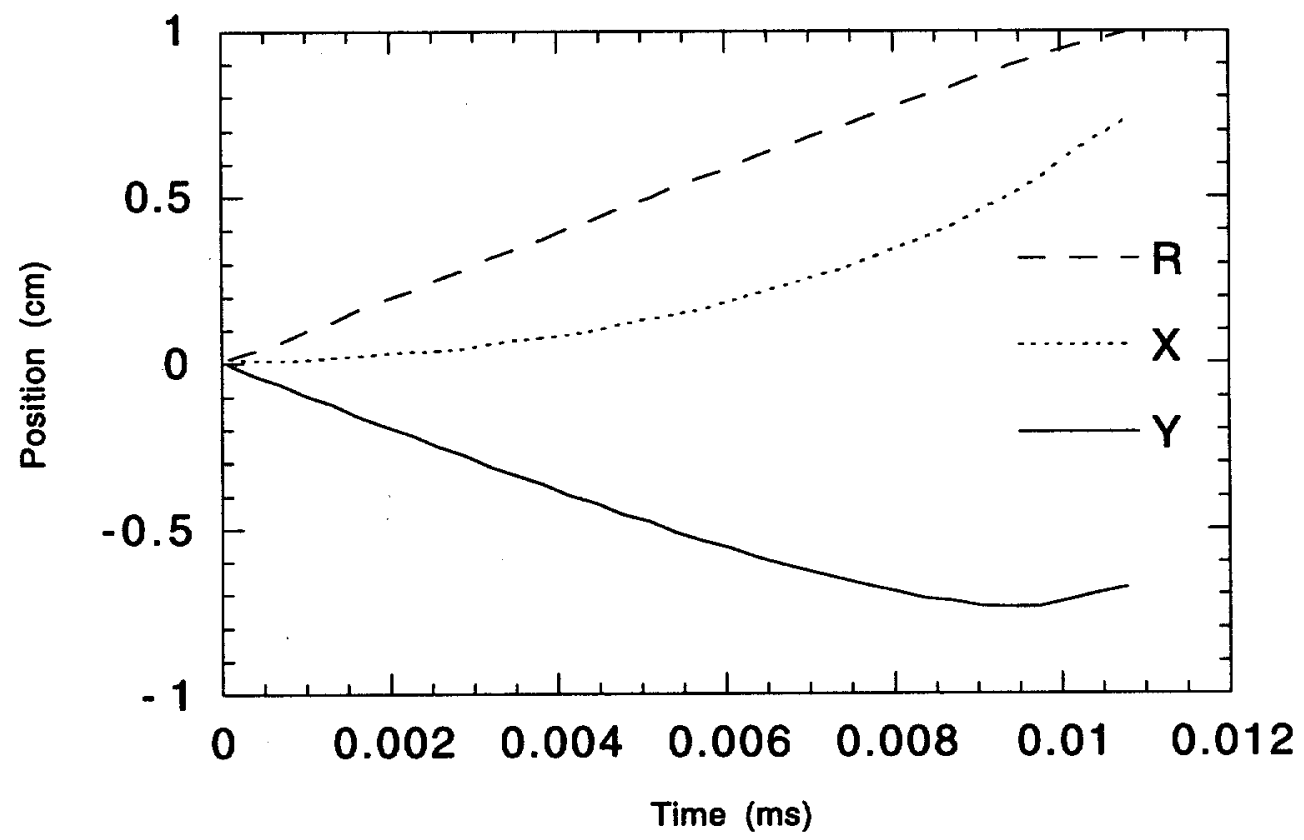

Figure c.3 Electron ExB drift in the presence of a large field error, $E_{x}=1 \mathrm{~V} / \mathrm{cm}$. The drift rate matches the predicted value of $105 \mathrm{~cm} / \mathrm{s}$.

shifts the equipotential, but still results in a closed orbit path. Fig. C.3 shows the deconfinement caused by a large field $E_{x}=1 \mathrm{~V} / \mathrm{cm}$. In this case, the radial field cannot compensate, no closed equipotential exists in the trap, and the particle drifts quickly to the wall. The drift velocity of $10^{5} \mathrm{~cm} / \mathrm{s}$ matches the prediction,

$$
v_{E \times B}=c \frac{E}{B}=3 \times 10^{10} \mathrm{~cm} / \mathrm{s}\left(\frac{\frac{1}{300} \text { statvolts } / \mathrm{cm}}{1000 \mathrm{Gs}}\right) .
$$

Fig. C. 4 shows the large-error case, with the addition of an applied 


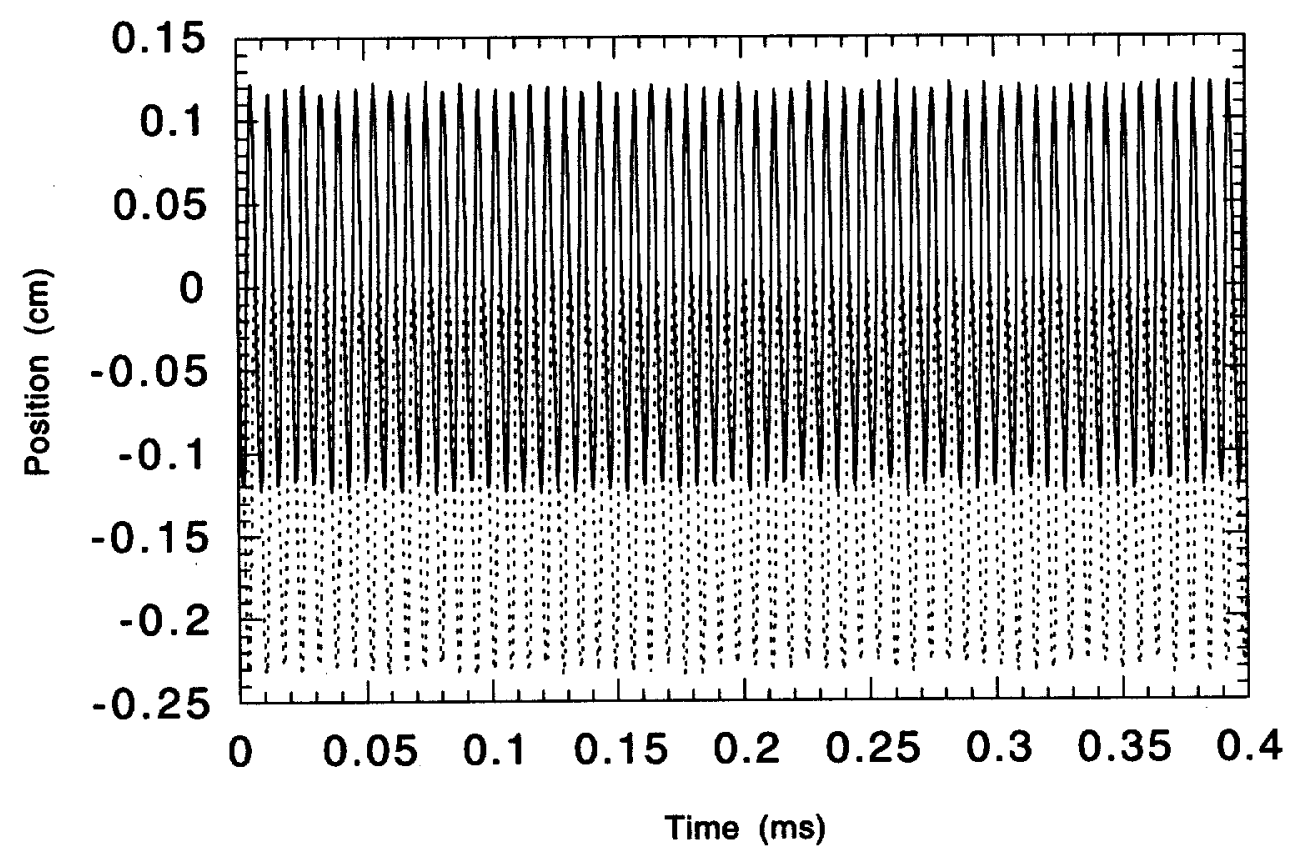

Figure c.4 Large electric field error $E_{x}=1 \mathrm{~V} / \mathrm{cm}$, stabilized by applied radial field $E_{r}=10 \mathrm{r} V / \mathrm{cm}$. This field strength is easily achieved in a nonneutral plasma. Dotted line is $x$ position, solid line is $y$ position.

radial field $E_{r}=10 r \mathrm{~V} / \mathrm{cm}$, such as would be found in a uniform plasma density $n=1.4 \times 10^{8} \mathrm{~cm}^{-3}$. Once again, the drift is canceled. This calculation does not truly simulate a plasma, because the charge of the plasma itself would be subject to the same drifts as the test particle, and it is not clear a priori that the dynamics of the motion would result in drift compensation.

Plasma confinement in the presence of large electric field errors has 
been studied at UCB [72]. The plasma is found to expand rapidly until the outer surface reaches the last closed equipotential curve, where the radial field produced by the plasma and electrodes exactly compensates for the applied error. The shape of the plasma then conforms to the equipotential, even if the curve is not perfectly circular. Particles are lost as collisions or other transport moves them across the separatrix to an open equipotential line.

\section{C.3 Magnetic Field Errors}

Magnetic field errors are implemented as a field curvature of given radius. As discussed in Sec. 2.4 .2 , the actual trap solenoid appears to be curved with a major radius $R=1 \mathrm{~km}$. Fig. C.5 shows the electron motion for curvature in the $x-z$ plane with $R=10 \mathrm{~m}$. Even the drift resulting from a curvature two orders of magnitude greater than the actual apparatus is completely compensated by the radial electric fields. Since $\mathrm{v}_{\mathrm{D}} \propto W(R B)^{-1}$, higher $B$ would also reduce the drift substantially, particularly when radiative cooling of the kinetic energy $W$ is considered. 


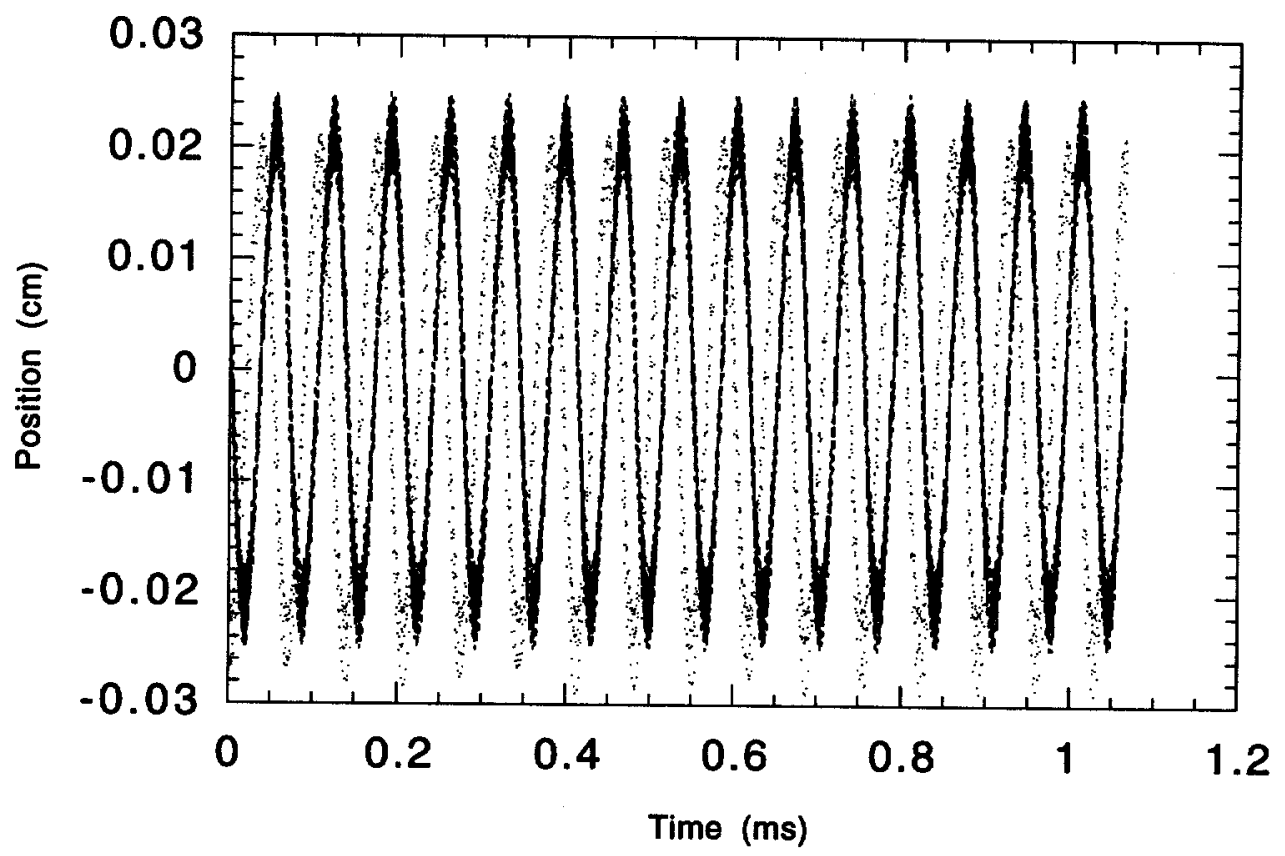

Figure c.5 Electron orbit in a curved magnetic field of radius $R=10 \mathrm{~m}$. The slight offset of $x$ (dots) relative to $y$ (solid) is due to the curvature offset of the orbit endpoint. The only apparent effect of the curvature is to increase the magnitude of the magnetron oscillation relative to Fig. c.1.

Fig. C.6 confirms that the ray-tracing code does in fact produce the curvature drift. In this case, the trap electrodes have been replaced by an infinite barrier at the reflection points of the previous calculations. The particle drifts to the wall, with a velocity of $230 \mathrm{~cm} / \mathrm{s}$. This is slower than the analytic prediction,

$$
\mathbf{v}_{\nabla}=\frac{\left(v_{1}^{2}+\frac{1}{2} v_{0}^{2}\right)}{R_{B} \Omega}\left(R_{B} \times B_{0}\right) \propto \frac{6.6 \times 10^{15} \mathrm{~cm}^{2} / \mathrm{s}^{2}}{(1000 \mathrm{~cm})\left(1.7 \times 10^{10} \mathrm{~s}^{-1}\right)} \hat{y}=400 \mathrm{~cm} / \mathrm{s}
$$

but is in the correct direction and of the correct order. 


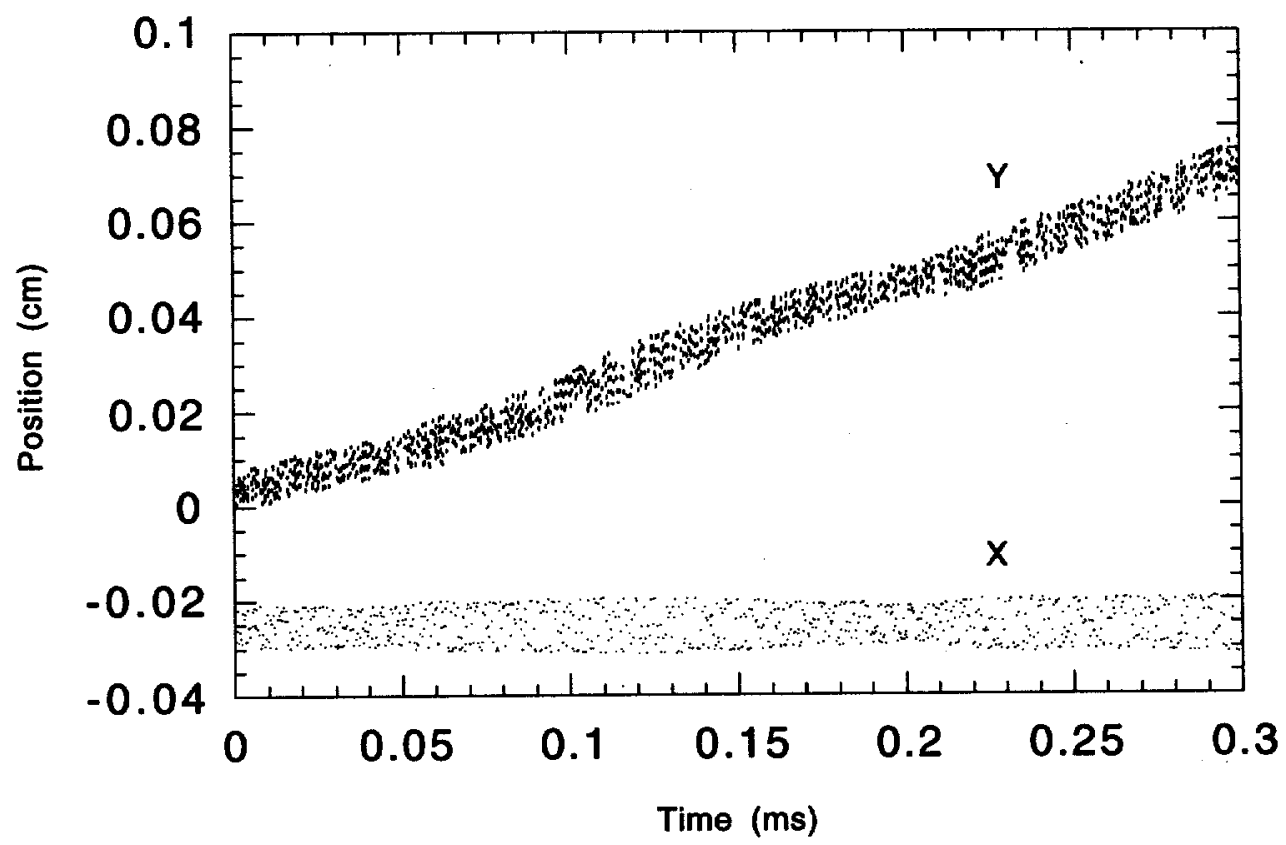

In summary, the single-particle ray-tracing demonstrates that radial electric fields can play a significant role in the confinement of particles in these traps. Even in long electrodes, the bounce-averaged radial electric fields in these electrodes are significant relative the fields from trapped charge. This suggests that the rotational stabilization may be an important part of both the length dependence and the density dependence of nonneutral plasma confinement. 


\section{References}

1. J. Scweppe, A. Gruppe, K. Bethge, H. Bokemeyer, T. Cowan, H. Folger, J. S. Greenberg, H. Brein, S. Ito, R. Schulé, D. Schwalm, K. E. Stiebing, N. Trautmann, P. Vincent, and M. Waldschmidt, Phys. Rev. Lett. 51, 2261 (1983).

2. T. Cowan, H. Backe, M. Begemann, K. Bethge, H. Bokemeyer, H. Folger, J. S. Greenberg, H. Grein, Y. Kido, M. Klüver, D. Schwalm, J. Schweppe, K. E. Stiebing, N. Trautmann, and P. Vincent, Phys. Rev. Lett. 54, 1761 (1985).

3. T. Cowan, H. Backe, K. Bethge, H. Bokemeyer, H. Folger, J. S. Greenber, K. Sakaguchi, D. Schwalm, J. Schweppe, K. E. Stiebing, P. Vincent, Phys. Rev. Lett. 56, 444 (1986).

4. T. E. Cowan, and J. S. Greenberg, in: Physics of Strong Fields, W. Greiner, ed. (Plenum Press, New York, 1987) p. 111.

5. Thomas Edward Cowan, Ph.D. Dissertation, Yale University, New Haven (1988).

6. H. Tsertos, C. Kozhuharov, P. Armbruster, P. Kienle, B. Krusche, and K. Schreckenbach, Phys. Rev. D. 40, 1397 (1989).

7. A. Scherdin, J. Reinhardt, W. Greiner, B. Muller, Rep. Prog. Phys. 
54, 1 (1991).

8. S. M. Judge, B. Krusche, K. Schreckenbach, H. Tsertos, and P. Kienle, Phys. Rev. Lett. 65, 972 (1990).

9. T. E. Cowan, R. H. Howell, R. R. Rohatgi, and J. Fajans, Nucl. Instrum. Methods B56/57, 599 (1991).

10. T. E. Cowan, J. H. Hartley, R. H. Howell, J. L. McDonald, R. R. Rohatgi, and J. Fajans, Materials Science Forum 105-110, 529 (1992).

11. J. H. Malmberg, T. M. O'Neil, A. W. Hyatt, and C. F. Driscoll, in Proceedings of the 1984 Sendai Symposium on Nonlinear Phenomena, eds. (Publisher, city,1984) p. 31.

12. T.E. Cowan, working notes, 1988-89.

13. J. D. Jackson, Classical Electrodynamics, 2nd Ed. (Wiley, New York, 1975), Chapter 13.

14. R. C. Davidson, Physics of Nonneutral Plasmas (Addison-Wesley, Redwood City, CA, 1990).

15. J. H. Malmberg and C. F. Driscoll, Phys. Rev. Lett. 44, 654 (1980). 
16. T. M. O'Neil and C. F. Driscoll, Phys. Fluids 22, 266 (1979).

17. S. A. Prasad and T. M. O'Neil, Phys. Fluids 22, 278 (1979).

18. N. A. Krall and A. W. Trivelpiece, Principles of Plasma Physics (San Francisco Press, San Francisco, 1986) p. 116.

19. J. H. Malmberg and T. M. O'Neil, Phys. Rev. Lett. 39, 1333 (1977).

20. T. M. O'Neil, Phys. Fluids 23, 2216 (1980).

21. J. H. Malmberg, C. F. Driscoll and W. D. White, Physica Scripta, T2, 288 (1982).

22. R. H. Cohen, Comments Plasma Phys. Cont. Fusion 4, 157 (1978).

23. C. F. Driscoll, K. S. Fine, and J. H. Malmberg, Phys. Fluids, 29, 2015 (1986).

24. C. F. Driscoll and J. H. Malmberg, Phys. Rev. Lett. 50, 167 (1983).

25. J. Fajans (private communication). 
26. C. F. Driscoll, in Low Energy Antimatter, ed. D. Cline (World Scientific, Singapore, 1986) p 189.

27. R. H. Howell, I. J. Rosenberg, and M. J. Fluss, Appl. Phys. A 43, 247 (1987).

28. D. M. Chen, K. G. Lynn, R. Pareja, and Bret Nielsen, Phys. Rev. D 31, 4123 (1985).

29. J. H. Malmberg and C. F. Driscoll, Phys. Rev. Lett. 44, 654 (1980).

30. Anthony Joseph Peurrung, Ph.D. Dissertation, University of California, Berkeley (1987).

31. J. R. Pierce, Theory and Design of Electron Beams (Van Nostrand, New York, 1954).

32. J.L. Wiza, Nucl. Instrum. Methods 162, 587 (1979).

33. E. H. Eberhardt, Appl. Opt. 18, 1418 (1979).

34. B.R. Beck, computer code CAMERA (Lawrence Livermore National Laboratory, Livermore, CA, 1994).

35. Kevin F. Fine, Ph.D. Dissertation, University of California, San 
Diego (1988).

36. B. R. Beck, J. Fajans, J. H. Malmberg, Phys. Plasmas 3, 1250 (1996).

37. Rajeev Rohatgi, computer code EввотT (Lawrence Livermore National Laboratory, Livermore, CA, 1992).

38. J. A. Notte, PhD thesis, UC Berkeley (1993).

39. B. R. Beck (personal communication).

40. B. R. Beck, computer code POISSON_BRB (Lawrence Livermore National Laboratory, Livermore, CA, 1997).

41. T. M. O'Neil and C. F. Driscoll, Phys. Fluids, 22, 266 (1979).

42. T. M. O'Neil, Phys Fluids 23, 725 (1980).

43. S. A. Prasad and T. M. O'Neil, Phys. Fluids 27, 206 (1984).

44. J. H. Malmberg and C. F. Driscoll, Phys. Rev. Lett. 44, 654 (1980).

45. Brian P. Cluggish, $\mathrm{Ph}$. D. dissertation, University of California, San Diego (1995). 
46. X.-P. Huang, F. Anderegg, E. M. Hollman, and C. F. Driscoll, Phys. Rev. Lett. 78, 875 (1997).

47. B. Ghaffari and R. S. Conti, Phys. Rev. Lett. 75,3118 (1995).

48. B. Ghaffari, PhD thesis, University of Michigan (1997).

49. I. Ahmad, et. al., Nucl. Phys. A 583, 247 (1995).

50. D. M. Larsen, Phys. Rev. A 20, 5217 (1979).

51. M. E. Glinsky and T. M. O'Neil, Phys. Fluids B 3, 1279 (1991).

52. V. Tsytovich and C. B. Wharton, Comments Plasma Phys. Cont. Fusion 4, 91 (1978).

53. Naoki Iwamoto, Phys. Rev. E. 47, 604 (1993).

54. G. P. Zank and R. G. Greaves, Phys. Rev. E 51, 6079 (1995).

55. D. P. Sheehan, R. McWilliams, and N. Rynn, Phys. Fluids B 5, 1523 (1993).

56. H. U. Rahman and M. Y. Yu, Phys. Rev. A 26, 1120 (1982). 
57. R. G. Greaves, M. D. Tinkle, and C. M. Surko, in Non-neutral Plasmas Physics II, eds. J. Fajans and D. H. E. Dubin (AIP, New York, 1995) p. 70.

58. M. H. Douglas and T. M. O'Neil, Phys. Fluids 21, 920 (1978).

59. D. R. Nicholson, Introduction to Plasma Theory (Krieger, Malabar, Florida, 1992) p 23.

60. R. Keinigs, Phys. Fluids 24, 860 (1981).

61. R. Keinigs, Phys. Fluids 27, 1427 (1984).

62. J. D. Crawford, T. M. O'Neil, and J. H. Malmberg, Phys. Rev. Lett. 54, 697 (1985).

63. J. D. Crawford, S. Johnston, A. N. Kaufman, and C. Oberman, Phys. Fluids 29, 3219 (1986).

64. J. D. Crawford and T. M. O'Neil, Phys. Fluids 30, 2076 (1987).

65. T. M. O'Neil, Phys. Rev. Lett. 55, 943 (1985).

66. D. H. E. Dubin and T. M. O'Neil, Phys. Rev. Lett. 78, 3868 (1997).

67. F. Anderegg, X.-P. Huang, E. M. Hollmann, C. F. Driscoll, T. M. 
O'Neil, and D. H. E. Dubin, Phys. Plasmas 4, 1552 (1997).

68. J. S. DeGrassie and J. H. Malmberg, Phys. Fluids 23, 63 (1980).

69. A. C. Cass, B. Cluggish, F. Anderegg, K. S. Fine, C. F. Driscoll, and E. Sarid, Bull. Am. Phys. Soc. 39, 1737 (1994).

70. D. L. Eggleston, J. D. Crawford, T. M. O'Neil, and J. H. Malmberg, in Plasma Physics and Controlled Nuclear Fusion Research 1986 (IAEA, Vienna, 1987) pp. 337-342.

71. D. L. Eggleston and J. H. Malmberg, Phys. Rev. Lett. 59, 1675 (1987).

72. John Anthony Notte IV, Ph.D. Dissertation, University of California, Berkeley (1993).

73. F. J. Wysocki, M. Leventhal, A. Passner and C. M. Surko, Hyperfine Interactions 44, 185 (1988).

74. C. M. Surko, M. Leventhal, A. Passner, and F. J. Wysocki, in Nonneutral Plasma Physics, eds. C. W. Roberson and C. F. Driscoll (AIP, New York 1988).

75. R. G. Greaves, M. D. Tinkle, and C. M. Surko, Phys. Plasmas 1, 1439 (1994). 
76. T. Akahane, T. Chiba, N. Shiotani, S. Tanigawa, T. Mikado, R. Suzuki, and M. Chiwaki, in Positron Annihilation (World Scientific, Singapore, 1989) p. 592.

77. J. Paridaens, D. Segers, M. Dorikens, and L. Dorikens-Vanpraet, in Materials Science Forum, 105-110, 1951 (1992).

78. D. W. Gidley and P. G. Coleman, in Positron Scattering in Gases (Plenum, New York, 1984) p. 66.

79. O. Sueoka, in Proc. Of Nato Advanced Research Workshop on Atomic Physics with Positrons, eds. J. W. Humberston and E. A. G. Armour (Plenum, NY 1987) pp 41-54.

80. O. Sueoka, and S. Mori, J. Phys. Soc. Jpn., 53, 2491 (1984).

81. K. R. Hoffman, M. S. Dababneh, Y.-F. Hsieh, W. E. Kaupilla, V. Pol, J. H. Smart, and T. S. Stein, Phys. Rev. A, 25, 1393 (1982).

82. T. J. Murphy and C. M. Surko, Phys. Rev. A, 46, 5696 (1992).

83. T. E. Cowan and J. H. Hartley, computer code PTRACE (Lawrence Livermore National Laboratory, Livermore, CA, 1997).

84. Lloyd M. Young and James H. Billen, computer code 
POISSON/SUPERFISH (Los Alamos National Laboratory, Los Alamos, NM, 1996). 


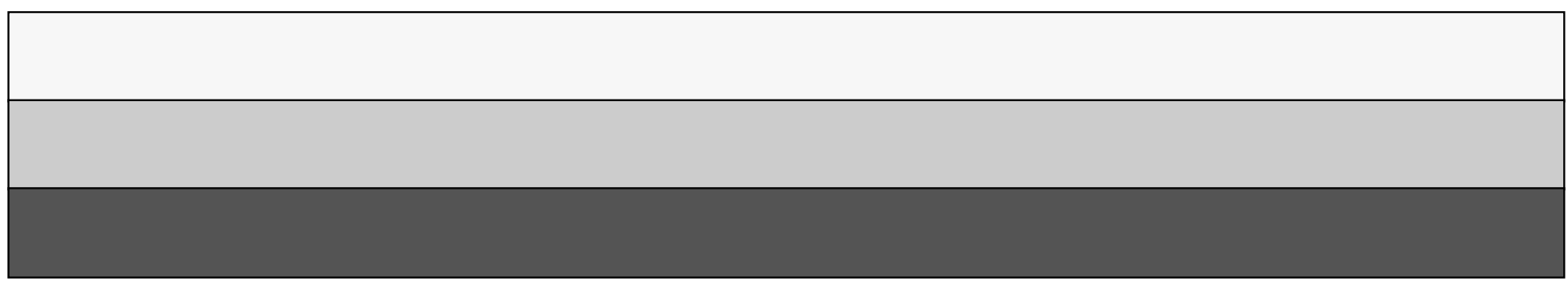

